\title{
EQUILIBRIUM MEASURES FOR THE HÉNON MAP AT THE FIRST BIFURCATION: UNIQUENESS AND GEOMETRIC/STATISTICAL PROPERTIES
}

\author{
SAMUEL SENTI AND HIROKI TAKAHASI
}

\begin{abstract}
For strongly dissipative Hénon maps at the first bifurcation parameter where the uniform hyperbolicity is destroyed by the formation of tangencies inside the limit set, we establish a thermodynamic formalism, i.e., prove the existence and uniqueness of an invariant probability measure which minimizes the free energy associated with a non continuous geometric potential $-t \log J^{u}$, where $t \in \mathbb{R}$ is in a certain large interval and $J^{u}$ denotes the Jacobian in the unstable direction. We obtain geometric and statistical properties of these measures.
\end{abstract}

\section{INTRODUCTION}

It is a well-known fact that unfoldings of non-transverse intersections between stable and unstable manifolds unleash surprisingly rich arrays of complicated behaviors (see, e.g., [27] and the references therein). Advancing our knowledge of such complexities is essential for understanding the realm of dynamics beyond uniform hyperbolicity.

In dimension two, an important role is played by the Hénon family

$$
f_{a}:(x, y) \mapsto\left(1-a x^{2}+\sqrt{b} y, \pm \sqrt{b} x\right), \quad 0<b \ll 1 .
$$

Indeed, a perturbation of this family is embedded in generic unfoldings of quadratic homoclinic tangencies associated with dissipative saddles of surface diffeomorphisms [26, 27]. Hence, a thorough study of the Hénon family should provide a general account on complexities unleashed by homoclinic tangencies in dimension two.

Another important feature of the Hénon family is that it describes a transition from Smale's horseshoe to the strange attractors of Benedicks \& Carleson [3]. For sufficiently large $a$, the non-wandering set of $f_{a}$ is a uniformly hyperbolic horseshoe [13]. As $a$ decreases, the stable and unstable directions get increasingly confused, until one reaches the first bifurcation parameter $a^{*}$ near 2. At $a=a^{*}$ the horseshoe undergoes a homoclinic (or heteroclinic) bifurcation, i.e., $\left\{f_{a}\right\}$ generically unfolds a quadratic tangency at $a=a^{*}$ between stable and unstable manifolds of the two fixed saddles [1, 2] (see FIGURE 1). On the other hand, close to and at the left of $a^{*}$ there exists a positive measure set of $a$-values corresponding to maps which admit nonuniformly hyperbolic strange attractors [3]. Despite the importance of this transition, many of its aspects are poorly understood, apart from a few partial results [31, 42].

In this paper we study the dynamics of $f_{a^{*}}$ from the viewpoint of ergodic theory and thermodynamic formalism. Write $f$ for $f_{a^{*}}$, and let $\Omega$ denote the non-wandering set of $f$. This set is closed, bounded and hence compact. Let $\mathcal{M}(f)$ denote the space of all $f$-invariant Borel probability measures endowed with the topology of weak convergence. For a potential

Date: November 7, 2018.

2010 Mathematics Subject Classification. 37D25, 37D35, 37D45. 

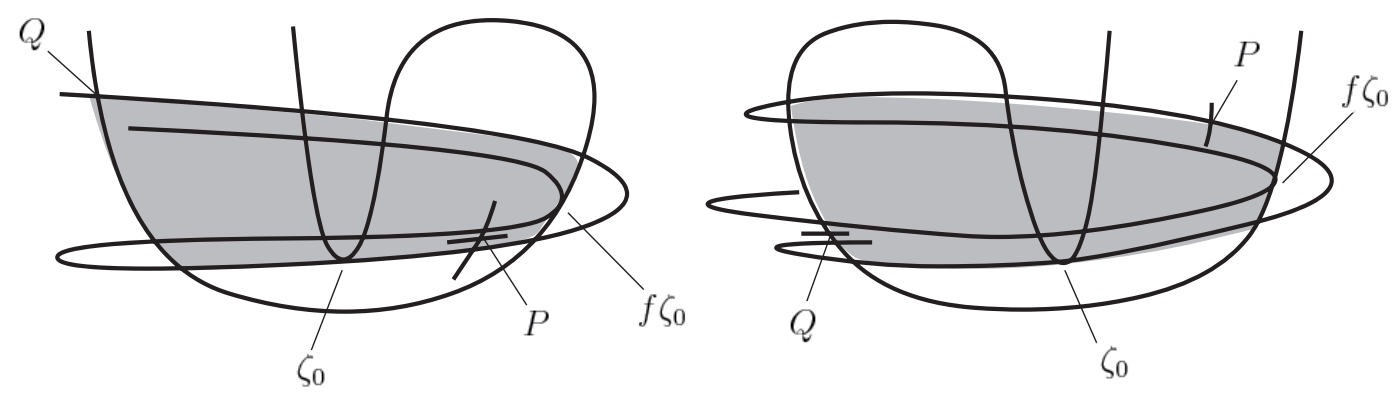

Figure 1. Manifold organization for $a=a^{*}$. There exist two hyperbolic fixed saddles $P, Q$ near $(1 / 2,0),(-1,0)$ correspondingly. In the orientation preserving case (left), $W^{u}(Q)$ meets $W^{s}(Q)$ tangentially. In the orientation reversing case (right), $W^{u}(P)$ meets $W^{s}(Q)$ tangentially. The shaded regions represent the region $R$ (see Sect 3.2$)$.

function $\varphi: \Omega \rightarrow \mathbb{R}$ the associated (minus of the) free energy function $F_{\varphi}: \mathcal{M}(f) \rightarrow \mathbb{R}$ is given by

$$
F_{\varphi}(\mu):=h(\mu)+\mu(\varphi),
$$

where $h(\mu)$ denotes the entropy of $\mu$ and $\mu(\varphi)=\int \varphi d \mu$. An equilibrium measure for the potential $\varphi$ is a measure $\mu_{\varphi} \in \mathcal{M}(f)$ which maximizes $F_{\varphi}$, i.e.

$$
F_{\varphi}\left(\mu_{\varphi}\right)=\sup \left\{F_{\varphi}(\mu): \mu \in \mathcal{M}(f)\right\} .
$$

The main example of potential functions we are concerned with is the family of potentials

$$
\varphi_{t}:=-t \log J^{u} \quad t \in \mathbb{R},
$$

where $J^{u}$ denotes the Jacobian along the unstable direction which is defined as follows. At a point $z \in \Omega$, let $E_{z}^{u}$ denote the one-dimensional subspace such that

$$
\varlimsup_{n \rightarrow \infty} \frac{1}{n} \log \left\|D_{z} f^{-n} \mid E_{z}^{u}\right\|<0 .
$$

Since $f^{-1}$ expands area, $E_{z}^{u}$ is unique when it makes sense. We call $E_{z}^{u}$ the unstable direction at $z$ and define $J^{u}(z):=\left\|D_{z} f \mid E_{z}^{u}\right\|$. It was proved in [39, Proposition 4.1] that $E^{u}$ makes sense for all $z \in \Omega$, and is continuous except at the fixed saddle $Q$ near $(-1,0)$.

The (non-uniform) expansion along the unstable direction is responsible for the chaotic behavior. Therefore, information on the dynamics of $f$ as well as the geometry of $\Omega$ is obtained by studying equilibrium measures for $\varphi_{t}$, and the associated pressure function $t \in \mathbb{R} \mapsto P(t)$, where

$$
P(t):=\sup \left\{F_{\varphi_{t}}(\mu): \mu \in \mathcal{M}(f)\right\} .
$$

Since $\varphi_{t}$ is merely bounded measurable, the existence of equilibrium measures for $\varphi_{t}$, let alone the uniqueness, is an issue. The existence was studied in [39]. We are now concerned with the 
existence and uniqueness of equilibrium measures for $\varphi_{t}$, and their geometric and statistical properties.

Theorem A. For any bounded interval $I \subset(-1, \infty)$ there exists $b_{0}>0$ such that if $0<b<b_{0}$, then for all $t \in I$ there exists a unique equilibrium measure for $\varphi_{t}$.

Several remarks are in order on Theorem A. Since entropies of invariant probability measures are written as linear combinations of the entropies of the ergodic components, and the same property holds for unstable Lyapunov exponents, the equilibrium measures in Theorem A must be ergodic. In addition, from our construction, they are supported on $\Omega$, i.e., give positive weight to any open set intersecting $\Omega$.

It was proved in [39, Theorem] that equilibrium measures for $\varphi_{t}$ exist for all negative $t$ and some (many) positive $t$. We cannot rule out the possibility of the coexistence of multiple equilibrium measures for $t \leq-1$, as is the case for the Chebyshev quadratic polynomial $x \in[-1,1] \rightarrow 1-2 x^{2}$. This is the reason why assume $t>-1$.

There are still few results concerning the thermodynamics of the Hénon maps. All currently known results in this direction are concerned with positive Lebesgue measure sets of parameters (close to but not containing $a^{*}$ ) for which the corresponding maps exhibit strange attractors [3, 8, 9, 26, 44]. For these parameters, SRB measures are constructed and shown to be unique in [6] (see also [8]). In our terms, these measures are equilibrium measures for $\varphi_{t}$ with $t=1$. The existence of equilibrium measures for continuous potentials is established in [44, and in particular, measures of maximal entropy exist. These are equilibrium measures for $\varphi_{t}$ with $t=0$. The uniqueness of measures of maximal entropy for a positive Lebesgue measure set of parameters is proved in [9]. The existence of equilibrium measures for $\varphi_{t}$ with $t$ other than 0,1 is not known.

The construction used in the proof of Theorem A allows us to characterize the Hausdorff dimension of a (one dimensional) unstable slice of $\Omega$ as the first zero of the pressure (see also [23, 24, 43]). Given a $C^{1}$ one-dimensional submanifold $\gamma$ of $\mathbb{R}^{2}$ and $p \in(0,1]$, the Hausdorff $p$-measure of a set $A \subset \gamma$ is given by

$$
m_{p}(A)=\lim _{\delta \rightarrow 0}\left(\inf \sum_{U \in \mathcal{U}} \ell(U)^{p}\right) .
$$

Here, $\ell$ denotes the diameter with respect to the induced metric on $\gamma$, and the infimum is taken over all coverings $\mathcal{U}$ of $A$ by open sets in $\gamma$ with diameter $\leq \delta$. The Hausdorff dimension of $A$ on $W^{u}(P)$, simply denoted by $\operatorname{dim}_{H}^{u}(A)$, is the unique number in $[0,1]$ such that

$$
\operatorname{dim}_{H}^{u}(A)=\sup \left\{p: m_{p}(A)=\infty\right\}=\inf \left\{p: m_{p}(A)=0\right\} .
$$

The pressure function $t \mapsto P(t)$ is convex, and so continuous. One has $P(0)>0$, and Ruelle's inequality 33 gives $P(1) \leq 0$. Since $f$ has no SRB measure [42, $P(1)<0$ holds. Hence the equation $P(t)=0$ has a unique solution in $(0,1)$, which we denote by $t^{u}$.

Theorem B. For any open set $\gamma$ in the unstable manifold of the fixed saddles of with $\gamma \cap \Omega \neq \emptyset$, we have $\operatorname{HD}(\gamma \cap \Omega)=t^{u}$. In addition, $t^{u} \rightarrow 1$ as $b \rightarrow 0$.

Our results are similar in spirit to the ones of Leplaideur and Rios [22, 23], in which a thermodynamic formalism for certain horseshoes with three branches and a single orbit of tangency was established (also see [21]). Certain hypotheses in [22, 23] on expansion/contraction rates and curvatures of invariant manifolds near the tangency are no longer true in our setting due 
to its strong dissipation. Our approach here is to take advantage of this strong dissipation, as in the study of Hénon-like systems [3, 7, 26, 44].

The construction and study of many relevant invariant measures can be carried out on the symbolic level, when a coding of the orbits into symbolic sequences is available. For uniformly hyperbolic systems, Markov partitions are used to code orbits with symbolic sequences over a finitely alphabet. The existence and uniqueness of equilibrium measures for Hölder continuous potentials were established in [10, 34, 40]. However, the map $f$ lacks such a nice partition. Indeed, the natural partition of $\Omega$ into the "left" and the "right" of the point of tangency near the origin, constructed in [39] only defines a semi-conjugacy between $f \mid \Omega$ and the full shift on two symbols. In order to avoid the discontinuity of $\varphi_{t}$ at $Q$, we must consider a (non-compact) subset of $\Omega$ which does not contain $Q$. We code the dynamics on this subset with a countable alphabet to establish the uniqueness (countable partitions were also constructed in [16, 23] albeit for other purposes/maps).

Our strategy for proving the uniqueness of the equilibrium measures is to construct an invariant measure as a candidate, and then show that it is indeed a unique measure which maximizes $F_{\varphi_{t}}$. The main step is to build an inducing scheme $(S, \tau)$. Here $S$ is a countable collection of pairwise disjoint Borel subsets of $\Omega$ called basic elements. The union of all basic elements is denoted by $X$, and $\tau$ is the first return time to $X$, which is constant on each basic element. The inducing scheme allows us to represent the first return map to $X$ as a countable (full) Markov shift. Under certain conditions on the potential function, which are satisfied by $\varphi_{t}$ with $t \in\left(t_{-}, t_{+}\right)$, where $t_{-}<0<t_{+}$depend on $t^{u}$ (see (26) for the precise definition), one can construct a Gibbs measure in the shift space following [25, 38]. The interval $\left(t_{-}, t_{+}\right)$ of Theorem A can be chosen arbitrarily large in $(-1, \infty)$ since $t_{-} \rightarrow-1$ and $t_{+} \rightarrow \infty$ as $t^{u}$ goes to 1 (c.f. Theorem B). This Gibbs measure is then used to obtain a unique invariant measure for the original system which minimizes the free energy among all measures which are liftable to the inducing scheme (i.e. those measures which can be obtained from symbolic shift invariant measures).

To show that the candidate measure is a unique equilibrium measure, one must show that non liftable measures (e.g., the Dirac measure at $Q$ ) do not maximize $F_{\varphi_{t}}$. This can be done in two steps. We first show that any ergodic measure with sufficiently large entropy is liftable to the inducing scheme $(S, \tau)$. We then show that, with some restriction on $t$, measures with small entropy do not maximize $F_{\varphi_{t}}$. In the second step we essentially use the fact that holonomy maps along stable manifolds are Lipschitz continuous. This is false in general, but true for $f$ as explained in Remark 3.5.

The construction of our inducing scheme is inspired by the work of Benedicks and Young [7] on Hénon-like strange attractors: points returning to a neighborhood of the tangency too fast, for which "long stable leaves" cannot be constructed, must be excluded from consideration. As a result, each basic element of the inducing scheme constructed here is Cantor-like. In addition, one must analyze its Hausdorff dimension, because Lebesgue almost every initial point diverges to infinity under positive iteration [42]. These factors make estimates more involved than [7].

We now move on to geometric and statistical properties. In what follows, let $\mu_{t}$ denote the equilibrium measure for $\varphi_{t}$ in Theorem A. We first give a characterization of $\mu_{t^{u}}$ in terms of dimension. To give a precise statement let us recall general facts on nonuniformly hyperbolic systems. Let $\mathcal{M}^{e}(f)$ denote the set of ergodic elements of $\mathcal{M}(f)$. Since any $\mu \in \mathcal{M}^{e}(f)$ has 
exactly one positive Lyapunov exponent [12], for $\mu$-a.e. $x \in \Omega$ the set

$$
W^{u}(x)=\left\{y \in \mathbb{R}^{2}: \varlimsup_{n \rightarrow \infty} \frac{1}{n} \log \left|f^{-n} x-f^{-n} y\right|<0\right\}
$$

is a smooth injectively immersed one-dimensional submanifold of $\mathbb{R}^{2}$ [28, 35]. We call $W^{u}(x)$ the unstable manifold of $x$. Let $\left\{\mu_{x}^{u}\right\}_{x \in \Gamma}$ denote the canonical system of conditional measures of $\mu$ along unstable manifolds [32]: $\mu_{x}^{u}$ is a probability measure supported on $W^{u}(x)$ such that $x \mapsto \mu_{x}^{u}(A)$ is measurable and $\mu(A)=\int \mu_{x}^{u}(A) d \mu(x)$ for any measurable set $A$. Let $\operatorname{dim}\left(\mu_{x}^{u}\right)$ denote the dimension of $\mu_{x}^{u}$, namely

$$
\operatorname{dim}\left(\mu_{x}^{u}\right)=\inf \left\{\operatorname{dim}_{H}^{u}(X): X \subset W^{u}(x), \mu_{x}^{u}(X)=1\right\} .
$$

Then, $\operatorname{dim}\left(\mu_{x}^{u}\right)$ is constant $\mu$-a.e. and this number is denoted by $\operatorname{dim}^{u}(\mu)$. We say $\mu \in \mathcal{M}^{e}(f)$ is a measure of maximal unstable dimension if

$$
\operatorname{dim}^{u}(\mu)=\sup \left\{\operatorname{dim}^{u}(\nu): \nu \in \mathcal{M}^{e}(f)\right\} .
$$

Theorem C. $\mu_{t^{u}}$ is the unique measure of maximal unstable dimension.

Considering the tower associated to the inducing scheme allows us to apply the result of Young [45] to deduce several statistical properties of $\mu_{t}$.

Theorem D. The following holds for $\left(f, \mu_{t}\right)$;

(1) for any $\eta \in(0,1]$ there exists $\tau \in(0,1)$ such that for any Hölder continuous $\phi: \Omega \rightarrow \mathbb{R}$ with Hölder exponent $\eta$ and $\psi \in L^{\infty}\left(\mu_{t}\right)$, there exists a constant $C(\phi, \psi)$ such that

$$
\left|\mu_{t}\left(\left(\varphi \circ f^{n}\right) \psi\right)-\mu_{t}(\varphi) \mu_{t}(\psi)\right| \leq C(\varphi, \psi) \tau^{n} \quad \text { for every } n>0
$$

(2) for any Hölder continuous $\phi: \Omega \rightarrow \mathbb{R}$ with $\int \phi d \mu_{t}=0$, there exists $\sigma \geq 0$ such that

$$
\frac{1}{\sqrt{n}} \sum_{i=0}^{n-1} \phi \circ f^{i} \longrightarrow \mathcal{N}(0, \sigma) \quad \text { in distribution, }
$$

where $\mathcal{N}(0, \sigma)$ is the normal distribution with mean 0 and variance $\sigma^{2}$. In addition, $\sigma>0$ if and only if $\phi \neq \psi \circ g-\psi$ for any $\psi \in L^{2}\left(\mu_{t}\right)$.

The rest of this paper consists of three sections. In Sect.2 we recall the general thermodynamical formalism for maps admitting inducing schemes from [30]. In Sect.3 we construct an efficient inducing scheme in the above sense. In Sect. 4 we define $t_{-}, t_{+}$and then check all the conditions on $\varphi_{t}, t \in\left(t_{-}, t_{+}\right)$, necessary for implementing the theory in Sect.2. This yields an $f$-invariant measure $\mu_{t}$ which maximizes $F_{\varphi_{t}}$ among all liftable measures. We show that $\mu_{t}$ is the unique measure which maximizes $F_{\varphi_{t}}$ among all measures. This completes the proof of Theorem A. Other theorems are also proved in Sect.4.

\section{Equilibrium MEASURES FOR MAPS ADMITTING INDUCING SCHEMES}

In this section we recall the construction of equilibrium measures for $\varphi$ developed in 30 . The main idea is to use an inducing scheme to relate the induced system to a countable Markov shift, and construct a Gibbs measure in the symbolic space for the induced potential following [25, 38]. Gibbs measures have integrable inducing time and are used to construct an equilibrium measure for the original map associated to the original potential function. 
2.1. Equilibrium states for countable Markov shifts. Denote the set of all bi-infinite sequences over a countable alphabet $S$ by

$$
S^{\mathbb{Z}}:=\left\{\underline{a}:=\left(\ldots, a_{-1}, a_{0}, a_{1}, \ldots\right): a_{i} \in S, i \in \mathbb{Z}\right\}
$$

and the (left full) shift by $\sigma: S^{\mathbb{Z}} \circlearrowleft$ i.e. $(\sigma(\underline{a}))_{i}=a_{i+1}$. Denote the cylinder sets by

$$
\left[b_{i}, \ldots, b_{j}\right]:=\left\{\underline{a} \in S^{\mathbb{Z}}: a_{k}=b_{k} \text { for all } i \leq k \leq j\right\} .
$$

Endow $S^{\mathbb{Z}}$ with the topology for which the cylinder sets form a base. The shift $\sigma$ is continuous with respect to this topology. Denote by $\mathcal{M}(\sigma)$ the collection of $\sigma$-invariant Borel probability measures on $S^{\mathbb{Z}}$. Given a function $\Phi: S^{\mathbb{Z}} \rightarrow \mathbb{R}$, let

$$
\mathcal{M}_{\Phi}(\sigma):=\{\nu \in \mathcal{M}(\sigma): \nu(\Phi)>-\infty\} .
$$

The $n^{\text {th }}$ variation of $\Phi$ is defined by

$$
V_{n}(\Phi):=\sup _{\left[b_{-n+1}, \ldots, b_{n-1}\right] \underline{a}, \underline{a}^{\prime} \in\left[b_{-n+1}, \ldots, b_{n-1}\right]}\left|\Phi(\underline{a})-\Phi\left(\underline{a}^{\prime}\right)\right| .
$$

The function $\Phi$ has strongly summable variation if

$$
\sum_{n \geq 1} n V_{n}(\Phi)<\infty
$$

The Gurevich pressure of $\Phi$ is defined by

$$
P_{G}(\Phi):=\lim _{n \rightarrow \infty} \frac{1}{n} \log \sum_{\sigma^{n}(\underline{a})=\underline{a}} \exp \left(\sum_{k=0}^{n-1} \Phi\left(\sigma^{k}(\underline{a})\right)\right) 1_{[b]}(\underline{a}),
$$

where $b \in S$. Since it depends only on the positive side of the sequences, one can prove (as in [36, Theorem 1]) that $P_{G}(\Phi)$ exists and is independent of $b$ whenever the variation

$$
V_{n}^{+}(\Phi):=\sup _{\left[b_{0}, \ldots, b_{n-1}\right] \underline{a}, \underline{a}^{\prime} \in\left[b_{0}, \ldots, b_{n-1}\right]}\left|\Phi(\underline{a})-\Phi\left(\underline{a}^{\prime}\right)\right|
$$

over all positive cylinders is summable: $\sum_{n \geq 1} V_{n}^{+}(\Phi)<\infty$. Also $P_{G}(\Phi)>-\infty$ holds in this case. We say $\nu_{\Phi} \in \mathcal{M}(\sigma)$ is a Gibbs measure for $\Phi$ if there exists a constant $C>0$ such that for any cylinder set $\left[b_{0}, \ldots, b_{n-1}\right]$ and any $\underline{a} \in\left[b_{0}, \ldots, b_{n-1}\right]$ we have

$$
C^{-1} \leq \frac{\nu_{\Phi}\left(\left[b_{0}, \ldots, b_{n-1}\right]\right)}{\exp \left(-n P_{G}(\Phi)+\sum_{k=0}^{n-1} \Phi\left(\sigma^{k}(\underline{a})\right)\right)} \leq C .
$$

Note that this definition only involves positive cylinders. We say $\nu_{\Phi} \in \mathcal{M}(\sigma)$ is an equilibrium measure for $\Phi$ if

$$
h_{\nu_{\Phi}}(\sigma)+\nu_{\Phi}(\Phi)=\sup _{\nu \in \mathcal{M}_{\Phi}(\sigma)}\left\{h_{\nu}(\sigma)+\nu(\Phi)\right\}
$$

The thermodynamics of the full shift $\sigma$ on the space of two-sided sequences over the countable alphabet $S$ is described in the following theorem from [30].

Proposition 2.1. [30] Let $\Phi: S^{\mathbb{Z}} \rightarrow \mathbb{R}$ be a potential function with $\sup \Phi<\infty$ and strongly summable variation. Then

(a) $P_{G}(\Phi)=\sup _{\nu \in \mathcal{M}_{\Phi}(\sigma)}\left\{h_{\nu}(\sigma)+\nu(\Phi)\right\}$;

(b) if $P_{G}(\Phi)<\infty$ then there exists a unique Gibbs measure $\nu_{\Phi}$ for $\Phi$;

(c) if $h_{\nu_{\Phi}}(\sigma)<\infty$ then $\nu_{\Phi} \in \mathcal{M}_{\Phi}(\sigma)$ and it is the unique equilibrium measure for $\Phi$. 
The main idea is to reduce the problem to the (left full) shift on the set of one-sided infinite sequences $S^{\mathbb{N}}$ by constructing a potential function cohomologous to the given potential $\Phi$ but which depends only on the positive coordinates of any point $\underline{a} \in S^{\mathbb{N}}$. The variational principle and the existence of a unique Gibbs and equilibrium measure for the one-sided shift and potential follows from [36, Theorem 3],[38, Theorem 1], [11, Theorem 1.1] (see also [25]). The statements of Proposition 2.1 follow by considering the natural extension of this one-sided Gibbs and equilibrium measure.

2.2. Gibbs and equilibrium measures for the induced map. From now on assume that $f$ is a continuous self map of finite topological entropy of a compact metric space $M$.

Definition 2.2. We say $f$ admits an inducing scheme $(S, \tau)$ of hyperbolic type. if there exist a countable collection $S$ of disjoint Borel subsets of $M$ called basic elements, and an inducing time function $\tau: S \rightarrow \mathbb{N}$ such that the following holds for the inducing domain $X:=\bigcup_{J \in S} J$ and the induced map $F: X \circlearrowleft$ defined by $F\left|J=f^{\tau(J)}\right| J$ for each $J \in S$.

(A1) $F(J)=f^{\tau(J)} J \subset X$ for each $J \in S$, and $f^{\tau(J)} \mid J$ extends to a homemorphism on $\bar{J}$;

(A2) for any $\underline{a}=\left\{J_{n}\right\}_{n \in \mathbb{Z}} \in S^{\mathbb{Z}}$, the coding map $h: S^{\mathbb{Z}} \rightarrow X^{*}:=\bigcup_{J \in S} \bar{J}$ given by

$$
h(\underline{a}):=\overline{J_{0}} \cap\left(\bigcap_{n \geq 1} f^{-\tau\left(J_{0}\right)} \circ \cdots \circ f^{-\tau\left(J_{n-1}\right)}\left(\overline{J_{n}}\right)\right) \cap\left(\bigcap_{n \geq 1} f^{\tau\left(J_{-1}\right)} \circ \cdots \circ f^{\tau\left(J_{-n}\right)}\left(\overline{J_{-n}}\right)\right)
$$

is well-defined. The restriction of $h$ to $S^{\mathbb{Z}} \backslash h^{-1}\left(X^{*} \backslash X\right)$ is a measurable bijection onto $X$ for which $F \circ h=h \circ \sigma$.

(A3) If $\nu$ is a $\sigma$-invariant Borel probability measure, then $\nu\left(h^{-1}\left(X^{*} \backslash X\right)\right)=0$.

In section 3.5 we construct an inducing scheme which satisfies conditions (A1)-(A3). Note that these conditions are stronger and thus imply the conditions of [30]. This is due to the fact that the inducing scheme is constructed over a first return time and that the boundary of the elements consists of stable manifolds of the fixed point $P$.

If $f$ admits an inducing scheme $(S, \tau)$ of hyperbolic type, the induced potential $\bar{\varphi}: X \rightarrow \mathbb{R}$ associated to a given potential $\varphi: M \rightarrow \mathbb{R}$ is defined by

$$
\bar{\varphi}:=\sum_{i=0}^{\tau-1} \varphi \circ f^{i}
$$

We say the induced potential $\bar{\varphi}$ has:

- (strongly) summable variations if $\Phi:=\bar{\varphi} \circ h$ has (strongly) summable variations;

- finite Gurevich pressure if $P_{G}(\Phi)<\infty$.

Let $\mathcal{M}(F)$ denote the set of $F$-invariant Borel probability measures on $X$ and $\mathcal{M}_{\bar{\varphi}}(F)=\{\nu \in$ $\mathcal{M}(F): \nu(\bar{\varphi})>-\infty\}$. An $F$-invariant probability measure $\nu_{\bar{\varphi}}$ is a Gibbs measure for $\bar{\varphi}$ if there exists an $\sigma$-invariant Gibbs measure $\nu_{\Phi}$ for $\Phi$ such that $\nu_{\bar{\varphi}}=h_{*} \nu_{\Phi}$. We call $\nu_{\bar{\varphi}}$ an equilibrium measure for $\bar{\varphi}$ if $\nu_{\bar{\varphi}} \in \mathcal{M}_{\bar{\varphi}}(F)$ and

$$
h_{\bar{\varphi}}(F)+\nu_{\bar{\varphi}}(\bar{\varphi})=\sup \left\{\nu \in \mathcal{M}_{\bar{\varphi}}(F): h_{\nu}(F)+\nu(\bar{\varphi})\right\} .
$$

By $(\mathrm{A} 2), h_{*}$ preserves entropy, the Gibbs property and integrals of potentials for measures supported on $S^{\mathbb{Z}} \backslash h^{-1}\left(X^{*} \backslash X\right)$. Additionally, $h^{-1}\left(X^{*} \backslash X\right)$ does not support any measures by Condition (A3). So the next statement is a direct consequence of Proposition 2.1. 
Corollary 2.3. Assume $f$ admits an inducing scheme $(S, \tau)$ of hyperbolic type and let $\varphi$ : $M \rightarrow \mathbb{R}$ be a potential with $\sup \bar{\varphi}<\infty$, strongly summable variations and finite Gurevich pressure. Then there exists a unique $F$-invariant Gibbs measure $\nu_{\bar{\varphi}}$ for $\bar{\varphi}$. If $h_{\nu_{\bar{\varphi}}}(F)<\infty$ then $\nu_{\bar{\varphi}} \in \mathcal{M}_{\bar{\varphi}}(F)$ and it is the unique equilibrium measure for $\bar{\varphi}$.

2.3. Candidate equilibrium measures for the original map. We now use the Gibbs measure for the induced map $F$ to construct an equilibrium measure for the original map $f$. For $\nu \in \mathcal{M}(F)$ with $\nu(\tau)<\infty$, the measure given by

$$
\mathcal{L}(\nu):=\left.\frac{1}{\nu(\tau)} \sum_{k=1}^{\infty}\left(f^{k}\right)_{*} \nu\right|_{\{\tau<k\}}
$$

is an $f$-invariant Borel probability measure. Let

$$
\mathcal{M}_{L}(f):=\{\mu \in \mathcal{M}(f): \exists \nu \in \mathcal{M}(F) \text { such that } \mathcal{L}(\nu)=\mu\} .
$$

Measures in $\mathcal{M}_{L}(f)$ are called liftable, and for $\mu \in \mathcal{M}_{L}(f)$ a measure $\nu$ with $\mathcal{L}(\nu)=\mu$ is called a lift of $\mu$.

Consider a potential $\varphi: M \rightarrow \mathbb{R}$, and let

$$
P_{L}(\varphi):=\sup \left\{h_{\mu}(f)+\mu(\varphi): \mu \in \mathcal{M}_{L}(f)\right\} .
$$

We say $\mu \in \mathcal{M}_{L}(f)$ is a candidate equilibrium measure for $\varphi$ if $F_{\varphi}(\mu)=P_{L}(\varphi)$. Candidate equilibrium measures are equilibrium measures in the classical sense if $P_{L}(\varphi)=\sup _{\mu \in \mathcal{M}(f)}\left\{h_{\mu}(f)+\mu(\varphi)\right\}$.

Abramov's and Kac's formulæ [29, Theorem 2.3] relate the entropy of $\mu$ and the integral of a potential $\varphi$ against $\mu$ to the entropy and the integral of the induced potential $\bar{\varphi}$ against a lift of $\mu$. Note that $F_{\varphi}(\mathcal{L}(\nu))=\frac{1}{\nu(\tau)} F_{\bar{\varphi}}(\nu)$ and so it is not straightforward that an equilibrium measure for $\bar{\varphi}$ projects to a candidate equilibrium measure for $\varphi$. However, this is the case for the equilibrium measure associated to the potential induced by $\varphi-P_{L}(\varphi)$ and the latter is cohomologous to $\varphi$. Observe that by [29, Theorem 4.2], the existence of a periodic point of $F$ implies that $\left|P_{L}(\varphi)\right|<\infty$ whenever $\varphi$ has summable variations and finite Gurevich pressure.

We say $\bar{\varphi}$ is positive recurrent if there exists $\eta_{0}>0$ such that

$$
P_{G}\left(\overline{\varphi-\left(P_{L}(\varphi)-\eta\right)}\right)<\infty \text { for all } 0 \leq \eta \leq \eta_{0}
$$

This condition implies positive recurrence condition in the sense of Sarig (c.f. [38]). Indeed, [29, Theorem 4.4] and the continuity of $P_{G}\left(\overline{\varphi-\left(P_{L}(\varphi)-\eta\right)}\right)$ with respect to $\eta$ for a positive recurrent potential $\bar{\varphi}$ imply $P_{G}\left(\overline{\varphi-P_{L}(\varphi)}\right)=0$. This implies the existence of some $N \in \mathbb{N}$ such that

$$
\inf _{n \geq N}\left\{\sum_{F^{n} x=x} \exp \left(\sum_{i=0}^{n-1} \overline{\varphi-P_{L}(\varphi)}\left(F^{i} x\right)\right)\right\}>0,
$$

which is equivalent to the positive recurrence condition of Sarig (c.f. [38, Theorem 1]).

With condition (A3) we obtain the following:

Proposition 2.4. 30](Existence and uniqueness of candidate equilibrium measures) Assume $f$ admits an inducing scheme $(S, \tau)$ of hyperbolic type. Let $\varphi: M \rightarrow \mathbb{R}$ be such that $\sup \overline{\varphi-P_{L}(\varphi)}<\infty$, and that $\varphi$ has strongly summable variations, finite Gurevich pressure and is positive recurrent. Then there exists a Gibbs measure $\nu$ for $\overline{\varphi-P_{L}(\varphi)}$. If $h_{\nu}(F)<\infty$, then $\nu \in \mathcal{M} \overline{\varphi-P_{L}(\varphi)}(F)$, and $\nu$ is the unique equilibrium measure for $\overline{\varphi-P_{L}(\varphi)}$. If $\nu(\tau)<\infty$, then $\mathcal{L}(\nu)$ is the unique candidate equilibrium measure for $\varphi$. 


\section{Construction of InduCing SCHEME}

In this section we construct an inducing scheme which will be used for the proof of the theorems. In Sect 3.1 we first state the existence of an inducing scheme with special properties (See Proposition 3.2). After preliminary geometric considerations in Sect 3.2, we construct in Sect. 3.3 and 3.4 a uniformly hyperbolic induced map with countably many branches. In Sect 3.5 we show how to obtain the inducing scheme from this induced map.

3.1. Inducing scheme. We start with preliminary definitions.

Definition 3.1. Let $\Gamma^{u}$ and $\Gamma^{s}$ be two families of compact $C^{1}$ curves such that:

- curves in $\Gamma^{s}$ are pairwise disjoint. Curves in $\Gamma^{u}$ are not necessarily pairwise disjoint;

- every $\gamma^{u} \in \Gamma^{u}$ intersects every $\gamma^{s} \in \Gamma^{s}$ at exactly one point;

- there is a minimum angle between $\gamma^{u}$ and $\gamma^{s}$ at the point of intersection;

Call the set

$$
\Lambda:=\left\{\gamma^{u} \cap \gamma^{s}: \gamma^{u} \in \Gamma^{u}, \gamma^{s} \in \Gamma^{s}\right\}
$$

a lattice defined by the families $\Gamma^{u}$ and $\Gamma^{s}$.

- $\Lambda^{\prime} \subset \Lambda$ is a u-sublattice of $\Lambda$ if there exists $\Gamma^{u^{\prime}} \subset \Gamma^{u}$ such that $\Lambda^{\prime}=\left\{\gamma^{u} \cap \gamma^{s}: \gamma^{u} \in\right.$ $\left.\Gamma^{u \prime}, \gamma^{s} \in \Gamma^{s}\right\}$. An s-sublattice of $\Lambda$ is defined similarly;

- $Q_{\Lambda^{\prime}} \subset \mathbb{R}^{2}$ is the rectangle spanned by $\Lambda^{\prime}$ if $\Lambda^{\prime} \subset Q_{\Lambda^{\prime}}$ and the boundary $\partial Q_{\Lambda^{\prime}}$ is made up of two non-intersecting curves in $\Gamma^{u^{\prime}}$ and two in $\Gamma^{s}$.

We now introduce a small constant $\varepsilon>0$ in order to quantify the proximity of $f$ to the Chebyshev quadratic polynomial $x \in[-1,1] \mapsto 1-2 x^{2}$. Set

$$
\sigma_{1}=2-\varepsilon \text { and } \sigma_{2}=4+\varepsilon .
$$

The next proposition states the existence of an inducing scheme with special properties.

Proposition 3.2. For any small $\varepsilon>0$ there exists $b_{0}>0$ such that if $0<b<b_{0}$, there exist a closed lattice $\Lambda$ defined by families $\Gamma^{u}$ and $\Gamma^{s}$, a collection $S$ of pairwise disjoint Borel subsets of $\Lambda$ and a function $\tau: S \rightarrow \mathbb{N}$ such that $(S, \tau)$ is an inducing scheme of $f=f_{a^{*}(b)}$ with the following properties:

(P1) (Topological structure) for each $J \in S, f^{\tau(J)} J \subset \bigcup_{J \in S} J$ and $f^{\tau(J)} \bar{J}$ is a u-sublattice of $\bigcup_{J \in S} \bar{J}$

(P2) (Backward contraction) there exist $C>0$ and $\lambda>1$ such that for each $\gamma^{u} \in \Gamma^{u}, z \in \gamma^{u}$ and $n>0,\left\|D_{f^{-n} z} f^{n} \mid T_{f^{-n} z} \gamma^{u}\right\| \geq C \lambda^{n}$

(P3) (Hyperbolicity)

(a) for each $\gamma^{u} \in \Gamma^{u}, J \in S$ and all $z \in \gamma^{u} \cap Q_{J}$,

$$
\sigma_{1}^{\tau(J)} \leq\left\|D_{z} f^{\tau(J)} \mid T_{z} \gamma^{u}\right\| \leq \sigma_{2}^{\tau(J)},
$$

where $\sigma_{1}, \sigma_{2}$ are the constants in (7);

(b) for each $\gamma^{s} \in \Gamma^{s}$ and all $z \in \gamma^{s}$,

$$
\left\|D_{z} f^{n} \mid T_{z} \gamma^{s}\right\| \leq(C b)^{\frac{n}{2}} \quad \forall n \geq 1,
$$

where $C>0$ is a constant independent of $\varepsilon$ and $b$;

(P4) (Distortion control) 
(a) for each $\gamma^{u} \in \Gamma^{u}$ and all $x, y \in \gamma \cap Q_{J}$,

$$
\log \frac{\left\|D_{x} f^{\tau(J)} \mid T_{x} \gamma^{u}\right\|}{\left\|D_{y} f^{\tau(J)} \mid T_{y} \gamma^{u}\right\|} \leq C\left|f^{\tau(J)} x-f^{\tau(J)} y\right|,
$$

where $C>0$ is a constant independent of $\varepsilon$ and $b$;

(b) for each $\gamma^{s} \in \Gamma^{s}$ and all $x, y \in \Lambda \cap \gamma^{s}$,

$$
\left\|D_{x} f^{n}\left|T_{x} \gamma^{u}\|\leq 2\| D_{y} f^{n}\right| T_{y} \gamma^{u}\right\| \quad \forall n \geq 1 ;
$$

(P5) Set $S(n):=\#\{J \in S: \tau(J)=n\}$. Then

$$
\varlimsup_{n \rightarrow \infty} \frac{1}{n} \log S(n) \leq \varepsilon ;
$$

(P6) any $\mu \in \mathcal{M}^{e}(f)$ with $h(\mu) \geq 2 \varepsilon$ is liftable (in the sense of Sect.2.3) to the inducing scheme $(S, \tau)$.

The rest of this section is entirely devoted to a proof of Proposition 3.2. Along the way we introduce large integers $\xi, N$ the purpose of which is as follows:

- $\xi$ determines the rate of approach of points in the lattice $\Lambda$ to critical zones around $\zeta_{0}$ (see (91)). We set

$$
\xi=\left[\frac{10}{\varepsilon}\right] ;
$$

- $N$ determines the size of a critical region $\Theta_{0}$ (See Sect 3.3 .)

For any given $\varepsilon$ as in the statement of Proposition 3.2 , we may choose sufficiently large $N$ at the expense of reducing $b$. Any generic positive constant which is independent of $\varepsilon, N, b$ is denoted by $C$.

3.2. The return map. For the Chebyshev quadratic polynomial $x \in[-1,1] \mapsto 1-2 x^{2}$, the first return map to $[-1 / 2,1 / 2]$ is uniformly expanding with controlled distortions [17]. We prove an analogous statement for $f$.

Recall that $P, Q$ denote the fixed saddles near $(1 / 2,0)$ and $(-1,0)$ respectively. If $f$ preserves orientation, let $W^{u}=W^{u}(Q)$. If $f$ reverses orientation, let $W^{u}=W^{u}(P)$. By a rectangle we mean any closed region bordered by two compact curves in $W^{u}$ and two in the stable manifolds of $P, Q$. By an unstable side of a rectangle we mean any of the two boundary curves in $W^{u}$. A stable side is defined similarly.

Denote by $\hat{\alpha}_{0}^{-}$the connected component of $W^{s}(Q) \cap\left\{(x, y) \in \mathbb{R}^{2}:|y| \leq b^{\frac{1}{4}}\right\}$ containing $Q$ and by $\hat{\alpha}_{0}^{+}$the connected component of $f^{-1} \hat{\alpha}_{0}^{-} \cap\left\{(x, y) \in \mathbb{R}^{2}:|y| \leq b^{\frac{1}{4}}\right\}$ which does not contain $Q$. Let $\gamma_{0}$ denote the compact curve in $W^{u}$ with endpoints in $\hat{\alpha}_{0}^{-}$and $\hat{\alpha}_{0}^{+}$which contains the saddle in $W^{u}$. Let $R$ denote the rectangle bounded by $f \gamma_{0}$ and $\hat{\alpha}_{0}^{ \pm}$(see FIGURE 1 ). One of the unstable sides of $R$ contains the point of tangency near $(0,0)$ denoted by $\zeta_{0}$ and $\alpha_{0}^{+}$ denote the stable side of $R$ which contains $f \zeta_{0}$. Let $\alpha_{0}^{-}$denote the other stable side of $R$.

Define a sequence $\left\{\tilde{\alpha}_{n}\right\}_{n \geq 0}$ of compact curves in $W^{s}(P) \cap R$ inductively as follows. First, let $\tilde{\alpha}_{0}$ be the component of $W^{s}(P) \cap R$ containing $P$. Given $\tilde{\alpha}_{n-1}$, define $\tilde{\alpha}_{n}$ to be one of the two components of $f^{-1} \tilde{\alpha}_{n-1} \cap R$ which is at the left of $\zeta_{0}$. Observe that $\left\{\tilde{\alpha}_{n}\right\}$ accumulates on $\alpha_{0}^{-}$from the right.

For each $n \geq 0, f^{-2} \tilde{\alpha}_{n} \cap R$ consists of four curves, two of them at the left of $\zeta_{0}$ and two at the right. Let $\alpha_{n+1}^{-}$denote the one which is not $\tilde{\alpha}_{n+2}$ and is at the left of $\zeta_{0}$. Among the two 


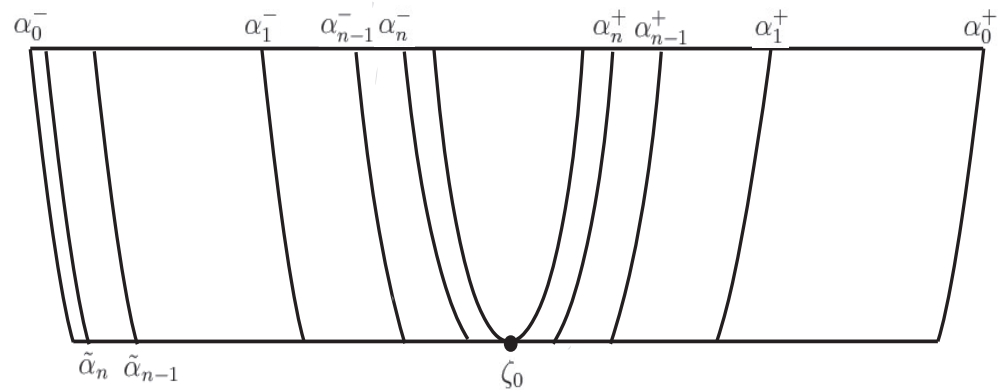

Figure 2. The rectangle $R$ and the curves $\left\{\tilde{\alpha}_{n}\right\},\left\{\alpha_{n}^{+}\right\},\left\{\alpha_{n}^{-}\right\}$. The $\left\{\tilde{\alpha}_{n}\right\}$ accumulate on the left stable side of $R$. Both $\left\{\alpha_{n}^{+}\right\}$and $\left\{\alpha_{n}^{-}\right\}$accumulate on the parabola in the stable manifold containing the point of tangency $\zeta_{0}$ near $(0,0)$.

at the right of $\zeta_{0}$, let $\alpha_{n+1}^{+}$denote the one which is at the left of the other. Then $\left\{\alpha_{n}^{-}\right\}$(resp. $\left\{\alpha_{n}^{+}\right\}$) accumulates the component of $W^{s}(Q) \cap R$ containing $\zeta_{0}$ from the left (resp. right). Observe that $\tilde{\alpha}_{1}=\alpha_{1}^{-}$and $\tilde{\alpha}_{0}=\alpha_{1}^{+}$. By definition, the curves obey the following diagram

$$
\left\{\alpha_{n+1}^{-}, \alpha_{n+1}^{+}\right\} \stackrel{f^{2}}{\rightarrow} \tilde{\alpha}_{n} \stackrel{f}{\rightarrow} \tilde{\alpha}_{n-1} \stackrel{f}{\rightarrow} \tilde{\alpha}_{n-2} \stackrel{f}{\rightarrow} \cdots \stackrel{f}{\rightarrow} \tilde{\alpha}_{1}=\alpha_{1}^{-} \stackrel{f}{\rightarrow} \tilde{\alpha}_{0}=\alpha_{1}^{+} .
$$

By a $C^{2}(b)$-curve we mean a compact, nearly horizontal $C^{2}$ curve such that the slopes of its tangent directions are $\leq \sqrt{b}$ and the curvature is everywhere $\leq \sqrt{b}$.

Given a $C^{2}(b)$-curve $\gamma$ with endpoints in $\bigcup_{n>1} \alpha_{n}^{+} \cup \alpha_{n}^{-}$, we define a partition $\mathcal{P}(\gamma)$ of $\gamma$ into $C^{2}(b)$-curves, by intersecting it with the countable families $\left\{\alpha_{n}^{+}\right\},\left\{\alpha_{n}^{-}\right\}$of pieces of stable manifolds. This is feasible since each of these pieces intersects $\gamma$ exactly one point (See [42, Remark 2.4]).

Let $\Theta$ denote the rectangle bordered by $\alpha_{1}^{-}, \alpha_{1}^{+}$and the unstable sides of $R$.

Lemma 3.3. ([39, Lemma 2.1]) Each connected component of $\Theta \cap W^{u}$ is a $C^{2}(b)$-curve.

Let $\gamma$ be a connected component of $\Theta \cap W^{u}$. For each $n>1$ there is a unique element of $\mathcal{P}(\gamma)$ with endpoints in $\alpha_{n}^{+}, \alpha_{n-1}^{+}$and a unique element with endpoints in $\alpha_{n-1}^{-}, \alpha_{n}^{-}$. No confusion will arise if we simplify notation by denoting both curves by $\gamma_{n}$.

Lemma 3.4. For any component $\gamma$ of $\Theta \cap W^{u}$ and each $\gamma_{n} \in \mathcal{P}(\gamma)$ the following holds:

(a) $f^{i} \gamma_{n} \subset \overline{R \backslash \Theta}$ for every $1 \leq i \leq n-1$;

(b) $f^{n} \gamma_{n}$ is a $C^{2}(b)$-curve in $\Theta$ with endpoints in the stable sides of $\Theta$ (See FIGURE 3).

Proof. We clearly have $f \gamma_{n} \subset \overline{R \backslash \Theta}$. Let $1<i \leq n$. The endpoints of $f^{i} \gamma_{n}$ are in $\tilde{\alpha}_{n-i+1}$, $\tilde{\alpha}_{n-i}$. [39, Lemma 2.1] implies that the sets $f^{i} \gamma_{n} \cap \tilde{\alpha}_{n-i+1}, f^{i} \gamma_{n} \cap \tilde{\alpha}_{n-i}$ are singleton. Since $f^{i} \gamma_{n} \subset R$, it follows that $f^{i} \gamma_{n}$ is contained in the rectangle bordered by $\tilde{\alpha}_{n-i+1}, \tilde{\alpha}_{n-i}$ and the unstable sides of $R$. Hence $f^{i} \gamma_{n} \subset \overline{R \backslash \Theta}$ and (a) holds. (b) follows from [39, Lemma 2.1] which states that any component of $\Theta \cap W^{u}$ is a $C^{2}(b)$-curve .

The next lemma, the proof of which is given in Appendix A1, states that $f^{n}$ expands tangent vectors of $\gamma_{n}$ uniformly, with controlled distortions.

Lemma 3.5. There exist $C>0$ and $N_{0}>0$ such that for any component $\gamma$ of $\Theta \cap W^{u}$ and each $\gamma_{n} \in \mathcal{P}(\gamma), n>N_{0}$ we have: 


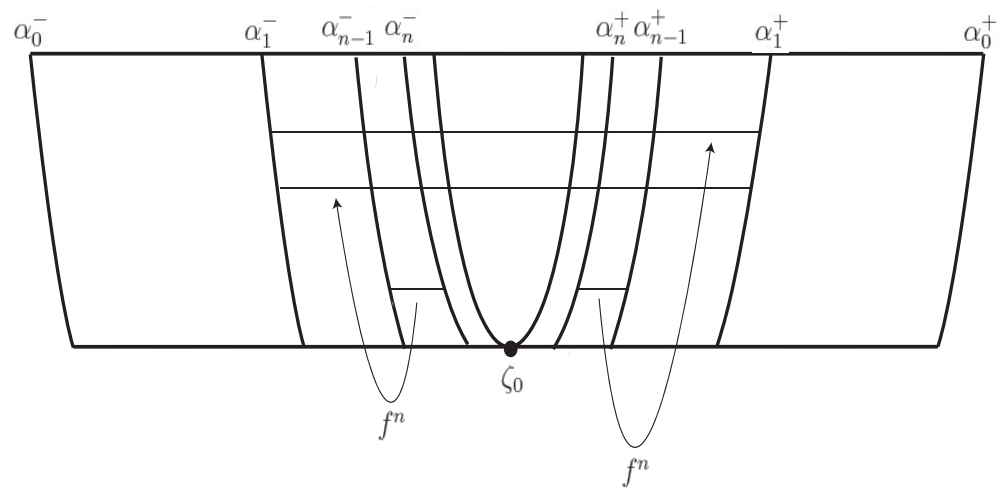

Figure 3. The partition element(s) $\gamma_{n}$ is mapped by $f^{n}$ to a $C^{2}(b)$-curve connecting the two stable sides of $\Theta$ (Lemma 3.4(b)).

(a) for all $x \in \gamma_{n}, \sigma_{1}^{n} \leq\left\|D_{x} f^{n} \mid E_{x}^{u}\right\| \leq \sigma_{2}^{n}$;

(b) for all $x, y \in \gamma_{n}, \log \frac{\left\|D_{x} f^{n} \mid E_{x}^{u}\right\|}{\left\|D_{y} f^{n} \mid E_{y}^{u}\right\|} \leq C\left|f^{n} x-f^{n} y\right|$.

Let $N \geq N_{0}$ (See Lemma 3.5), and let $\Theta_{0}=\Theta_{0}(N)$ denote the rectangle bordered by $\alpha_{N}^{-}$, $\alpha_{N}^{+}$and the unstable sides of $\Theta$. Returns to the inside of $\Theta_{0}$ are treated by Lemma 3.5.

3.3. Construction of the lattice $\Lambda$. We now construct a lattice $\Lambda$ defined by families $\Gamma^{u}$, $\Gamma^{s}$ of $C^{1}$ curves in $\Theta$.

Construction of $\Gamma^{u}$. Denote by $\tilde{\Gamma}^{u}$ the collection of connected components of $\Theta \cap W^{u}$. Define

$$
\Gamma^{u}=\left\{\gamma^{u}: \gamma^{u} \text { is the pointwise limit of a sequence in } \tilde{\Gamma}^{u}\right\} \text {. }
$$

Since elements of $\tilde{\Gamma}^{u}$ are $C^{2}(b)$-curves by Lemma 3.3, the pointwise convergence is equivalent to the uniform convergence. Since curves in $\tilde{\Gamma}^{u}$ are pairwise disjoint, the uniform convergence is equivalent to the $C^{1}$ convergence. Hence, curves in $\Gamma^{u}$ are $C^{1}$ and the slopes of their tangent directions are $\leq \sqrt{b}$.

Remark 3.1. Several remarks are in order on what intersections are possible for curves in $\Gamma^{u}$ (See FIGURE 4, and also [5, Lemma 4.3, Fig. 5] for comparison):

- each $\gamma^{u}$-curve is the (strictly) monotone limit of curves in $\tilde{\Gamma}^{u}$. For curves in $\Gamma^{u} \backslash \tilde{\Gamma}^{u}$, this follows from the definition. For those in $\tilde{\Gamma}^{u}$, this follows from the Inclination Lemma ([19, Proposition 6.2.23]). Hence, any connected component of the union of $\gamma^{u}$-curves contains at most two curves;

- two intersecting $\gamma^{u}$-curves are tangent at every point of the intersection;

- the backward contraction in Proposition 3.2(P2) and that $f$ contracts area imply the following: if $\gamma_{1}, \gamma_{2} \in \Gamma^{u}$ intersect each other, then $\gamma_{1} \cap \gamma_{2}$ is connected;

- this implies that there are at most countably many pairs that intersect each other.

Construction of $\Gamma^{s}$. For each $k \geq 0$ let $\Theta_{k}$ denote the rectangle bordered by $\alpha_{\xi k+N}^{-}, \alpha_{\xi k+N}^{+}$and the unstable sides of $\Theta$. Observe that

$$
\Theta \supset \Theta_{0} \supset \Theta_{1} \supset \Theta_{2} \supset \cdots .
$$


Let $\gamma^{u}\left(\zeta_{0}\right)$ denote the lower unstable side of $\Theta$, which contains the point $\zeta_{0}$ of tangency. Set $\Omega_{0}:=\overline{\gamma^{u}\left(\zeta_{0}\right) \backslash \Theta_{0}}$. For $n>0$ define the set of points whose recurrence rate to the region of tangency is slow

$$
\Omega_{n}:=\overline{\left\{z \in \gamma^{u}\left(\zeta_{0}\right) \cap \Omega_{n-1}: f^{n} z \in R \backslash \Theta_{n}\right\}}
$$

where $\xi$ is defined in (86). Set $\Omega_{\infty}:=\bigcap_{n \geq 0} \Omega_{n}$, which is the nonempty compact set (See Remark 3.4 below). The fact that the rate at which the orbit of points in $\Omega_{\infty}$ returns close to the homoclinic tangency is slow will be fundamental in proving that the first return map to the set $\Lambda$ exhibits the properties mentioned in Proposition 3.2).

Remark 3.2. Let $K=\left\{z \in \mathbb{R}^{2}:\left\{f^{n} z\right\}_{n \in \mathbb{Z}}\right.$ is bounded $\}$. It is immediate to check that $\Omega_{\infty} \subset K$. Since $K=\Omega$ [39, Section 3], $\Omega_{\infty} \subset \Omega$ holds.

By a vertical $C^{2}(b)$-curve we mean a compact, nearly vertical $C^{2}$ curve with endpoints in the unstable sides of $\Theta$, and of the form

$$
\left\{(x(y), y):\left|x^{\prime}(y)\right| \leq C \sqrt{b},\left|x^{\prime \prime}(y)\right| \leq C \sqrt{b}\right\} .
$$

A vertical $C^{2}(b)$-curve $\gamma^{s}$ is called a long stable leaf if for any $x, y \in \gamma^{s},\left|f^{n} x-f^{n} y\right| \leq(C b)^{\frac{n}{2}}$ holds for every $n \geq 0$. The next lemma is proved in Appendix A2. The angle $\angle(\cdot, \cdot)$ between two one-dimensional tangent spaces is given by the (smaller) angle between their basis vectors.

Lemma 3.6. For any $z \in \Omega_{\infty}$ there exists a unique long stable leaf $\gamma^{s}(z)$ through $z$. In addition, $\gamma^{s}(z) \subset \Theta$ and the following holds:

(a) if $f^{n}\left(\gamma^{s}\left(z_{1}\right)\right) \cap \gamma^{s}\left(z_{2}\right) \neq \emptyset$ for $n \geq 0$, then $f^{n} \gamma^{s}\left(z_{1}\right) \subset \gamma^{s}\left(z_{2}\right)$.

(b) $\left\|D f_{x}^{n}\left(\begin{array}{l}1 \\ 0\end{array}\right)\right\| \leq 2 \cdot\left\|D f_{y}^{n}\left(\begin{array}{l}1 \\ 0\end{array}\right)\right\|$ for all $x, y \in \gamma^{s}(z)$ and $n \geq 0$;

(c) if $x_{1} \in \gamma^{s}\left(z_{1}\right), x_{2} \in \gamma^{s}\left(z_{2}\right)$, then $\angle\left(T_{x_{1}} \gamma^{s}\left(z_{1}\right), T_{x_{2}} \gamma^{s}\left(z_{2}\right)\right) \leq C \sqrt{b}\left|x_{1}-x_{2}\right|$.

Define

$$
\Gamma^{s}=\left\{\gamma^{s}(z): z \in \Omega_{\infty}\right\}
$$

where $\gamma^{s}(z)$ is the long stable leaf through $z$ in Lemma 3.6.

3.4. Construction of an induced map on $\Lambda$. Consider the lattice $\Lambda$ defined by $\Gamma^{u}$ and $\Gamma^{s}$ : $\Lambda=\left\{\gamma^{u} \cap \gamma^{s}: \gamma^{u} \in \Gamma^{u}, \gamma^{s} \in \Gamma^{s}\right\}$. Let

$$
\mathcal{W}^{s}=\bigcup_{\gamma^{s} \in \Gamma^{s}} \gamma^{s}
$$

Define a first entry time $\tau: \Omega \rightarrow \mathbb{N} \cup\{\infty\}$ to $\Lambda$ by

$$
\tau(z)=\inf \left(\left\{n>0: f^{n} z \in \Lambda\right\} \cup\{\infty\}\right) .
$$

[39, Lemma 2.2] implies $\Omega \cap \mathcal{W}^{s} \subset \Lambda$, and so $\tau(z)=\inf \left(\left\{n>0: f^{n} z \in \mathcal{W}^{s}\right\} \cup\{\infty\}\right)$.

Lemma 3.7. There exists a collection $\mathcal{Q}$ of pairwise disjoint subsets of $\Omega_{\infty}$ such that:

(a) $\bigcup_{\omega \in \mathcal{Q}} \omega=\left\{z \in \Omega_{\infty}: \tau(z)<\infty\right\}$;

(b) $\tau$ is constant on each $\omega \in \mathcal{Q}$ not intersecting $\alpha_{1}^{-} \cup \alpha^{+}$(denote this value by $\tau(\omega)$. For all other $\omega \in \mathcal{Q}$, let $\tau(\omega)=2$ );

(c) for each $\omega \in \mathcal{Q}$ there exists $\gamma \in \tilde{\Gamma}^{u}$ such that $f^{\tau(\omega)} \bar{\omega}=\gamma \cap \mathcal{W}^{s}$.

(d) for each $\omega \in \mathcal{Q}, \bar{\omega} \backslash \omega \subset W^{s}(P) \backslash\left\{\alpha_{1}^{+}\right\}$. 


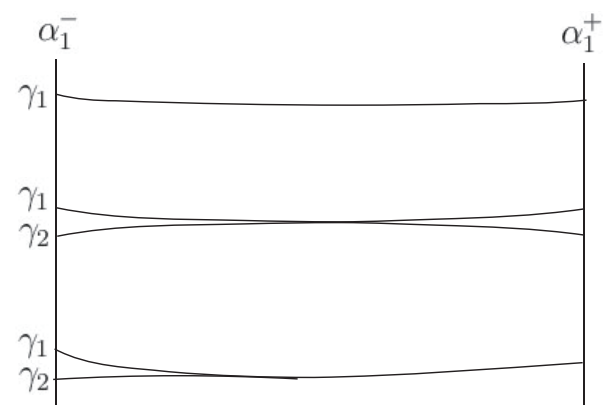

Figure 4. The curves in $\Gamma^{u}$.

We finish the construction of an induced map assuming the conclusions of Lemma 3.7. For each $\omega \in \mathcal{Q}$ in Lemma 3.7. consider the $s$-sublattice of $\Lambda$ defined by the families $\Gamma^{u}$ and $\left\{\gamma^{s}(z): z \in \omega\right\}$. Define $\hat{S}$ to be the collection of these $s$-sublattices of $\Lambda$. Let

$$
B=\{z \in \Omega: \tau(z)=\infty\},
$$

and set

$$
\Lambda_{B}:=\Lambda \cap B
$$

We do not know if $\Lambda_{B}=\emptyset$. Since elements of $\hat{S}$ are $s$-sublattices of $\Lambda$, so is $\Lambda_{B}$ unless it is an empty set. One can show that $\Lambda \backslash \Lambda_{B}$ is dense in $\Lambda$.

Remark 3.3. Proposition 3.2 establishes that the first return map of points of $\Lambda$ to itself has good hyperbolic and distortion properties, which allows us to apply the thermodynamical formalism. However, there are non-wandering points whose forward orbits never enter $\Lambda$. The set of all such points is denoted by $B$ and one needs to control its size (in terms of Hausdorff dimension) in order to show that it does not support any equilibrium measures with large entropy.

Corollary 3.8. The following holds:

(a) $\bigcup_{I \in \hat{S}} I=\Lambda \backslash \Lambda_{B}$;

(b) $\tau$ is constant on each $I \in \hat{S}$ not intersecting $\alpha_{1}^{-} \cup \alpha_{1}^{+}$(denote this value by $\tau(I)$. For all other $I \in \hat{S}$, let $\tau(I)=2)$;

(c) for each $I \in \hat{S}, f^{\tau(I)} \bar{I}$ is a u-sublattice of $\Lambda$;

(d) for each $I \in \hat{S}, \bar{I} \backslash I \subset W^{s}(P) \backslash\{P\}$.

Proof. From Proposition 3.7 and Lemma 3.6(a).

In order to prove Lemma 3.7 we need some preliminary considerations on the geometry of $\Omega_{\infty}$ and $\mathcal{W}^{s}$.

Definition 3.9. The set $\gamma^{u}\left(\zeta_{0}\right) \backslash \Omega_{0}$ is called a gap of order 0 . For each $n \geq 1$, any connected component of $\Omega_{n-1} \backslash \Omega_{n}$ is called a gap of $\Omega_{\infty}$ of order $n$.

For each $n \geq 0$, by a gap of $\mathcal{W}^{s}$ of order $n$ we mean any rectangle bordered by the closure of a gap of $\Omega_{\infty}$ of order $n$, a segment in the upper unstable side of $\Theta$, and two long stable leaves joining their endpoints.

Remark 3.4. By construction, the following holds: 

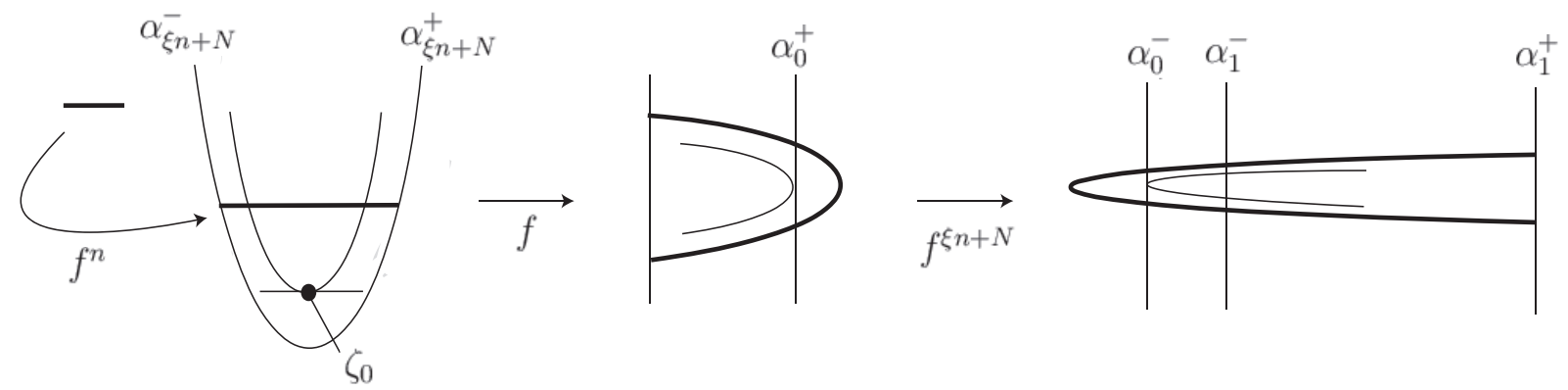

Figure 5. The closure of a gap $G$ of $\Omega_{\infty}$ of order $n$ is mapped by $f^{n}$ to a $C^{2}(b)$-curve connecting the two stable sides of $\Theta_{n}$. Hence $f^{n+1} G$ is folded. $f^{n+i} G \subset \overline{R \backslash \Theta}$ holds for every $1 \leq i \leq \xi n+N$.

- If $A$ is a component of $\Omega_{n-1}$, then the $f^{n}$-images of the endpoints of $A$ are contained in $\overline{R \backslash \Theta}$. In particular, any gap of $\Omega_{\infty}$ of order $n$ intersecting $A$ is strictly contained in $A$. Hence, $\Omega_{n} \neq \emptyset$ and as a result $\Omega_{\infty}$ is a nonempty compact set.

- the closure of each gap of $\Omega_{\infty}$ of order $n$ is sent by $f^{n}$ diffeomorphically onto a $C^{2}(b)$ curve connecting the two stable sides of $\Theta_{n}$ (See FIGURE 5).

- Each gap of $\mathcal{W}^{s}$ of order $n \geq 0$ is mapped by $f^{n}$ to a rectangle whose stable sides are in $\alpha_{\xi n+N}^{ \pm}$and whose unstable sides are $C^{2}(b)$-curves in $W^{u}$. (See FIGURE 6).

Lemma 3.10. Let $G$ be a gap of order $g$. Then for $0 \leq i \leq g, f^{i} G \cap \mathcal{W}^{s}=\emptyset$.

Proof. Suppose there exists a point $x \in f^{i} G \cap \mathcal{W}^{s} \neq \emptyset$ for some $0 \leq i \leq g$. Then $f^{g-i} x \in$ $\overline{R \backslash \Theta_{g-i}}$. On the other hand, $f^{g-i} x \in f^{g} G \subset \Theta_{g}$ and thus $f^{g-i} x \cap \overline{R \backslash \Theta_{g}}=\emptyset$, and $f^{g-i} x \cap$ $\overline{R \backslash \Theta_{g-i}}=\emptyset$, a contradiction.

Proof of Lemma 3.7. We construct $\mathcal{Q}$ by induction. Consider the partition $\mathcal{P}\left(\gamma^{u}\left(\zeta_{0}\right)\right)$ of $\gamma^{u}\left(\zeta_{0}\right)$ into $C^{2}(b)$-curves (See Sect 3.2 for the definition of this partition), and set $\mathcal{P}_{0}=\mathcal{P}\left(\gamma^{u}\left(\zeta_{0}\right)\right) \mid \Omega_{0}$. For each $\omega \in \mathcal{P}_{0}$ let $2 \leq \tau(\omega) \leq N$ denote the unique integer such that $f^{\tau(\omega)} \omega \subset \tilde{\Gamma}^{u}$. There are exactly two elements of $\mathcal{P}_{0}$ for which $\tau(\omega)=2$. If $\tau(\omega)=2$, then we let $f^{-\tau(\omega)}\left(f^{\tau(\omega)} \omega \cap \mathcal{W}^{s}\right) \in$ $\mathcal{Q}$. If $\tau(\omega)>2$, then we let $f^{-\tau(\omega)}\left(f^{\tau(\omega)} \omega \cap \mathcal{W}^{s} \backslash\left(\alpha_{1}^{-} \cup \alpha_{1}^{+}\right)\right) \in \mathcal{Q}$. We remove these pre-images of $\alpha_{1}^{-} \cup \alpha_{1}^{+}$to ensure that elements of $\mathcal{Q}$ are pairwise disjoint. The next sublemma justifies this construction.

Sublemma 3.11. For each $\omega \in \mathcal{P}_{0}, f^{-\tau(\omega)}\left(f^{\tau(\omega)} \omega \cap \mathcal{W}^{s}\right) \subset \Omega_{\infty}$.

Proof. Recall that $\Omega_{\infty}$ is the set of points which do not approach the critical region too close and too soon. In the proof below we use the fact that if a point in a $\gamma^{s}$-curve is in $\Omega_{\infty}$ then the curve satisfies the same property.

Let $z \in f^{-\tau(\omega)}\left(f^{\tau(\omega)} \omega \cap \mathcal{W}^{s}\right)$. Since $f^{-\tau(\omega)}\left(f^{\tau(\omega)} \omega \cap \mathcal{W}^{s}\right) \subset \Omega_{\tau(\omega)}$ by the definition of $\tau(\omega)$, to conclude $z \in \Omega_{\infty}$ it suffices to show $z \in \Omega_{n+\tau(\omega)}$ for every $n>0$.

There exists $y \in \Omega_{\infty}$ such that $f^{\tau(\omega)} z \in \gamma^{s}(y)$. Then $f^{n} y \in \overline{R \backslash \Theta_{n}}$ by (91). Suppose that $f^{n+\tau(\omega)} z \notin \overline{R \backslash \Theta_{n}}$, namely $f^{n+\tau(\omega)} z \in \Theta_{n}$. Then $f^{n} \gamma^{s}(y)$ would intersect the stable side of $\Theta_{n}$. Also, since $y \in \Omega$ the forward iterates of $\gamma^{s}(y)$ are contained in a bounded region, and so $f^{n} \gamma^{s}(y) \subset R$. Because of the contraction along $\gamma^{s}(y)$ and since the stable sides of $\Theta_{n}$ are contained in $W^{s}(P)$, this would imply $f^{n} \gamma^{s}(y) \subset W^{s}(P)$. However, it is not possible to 


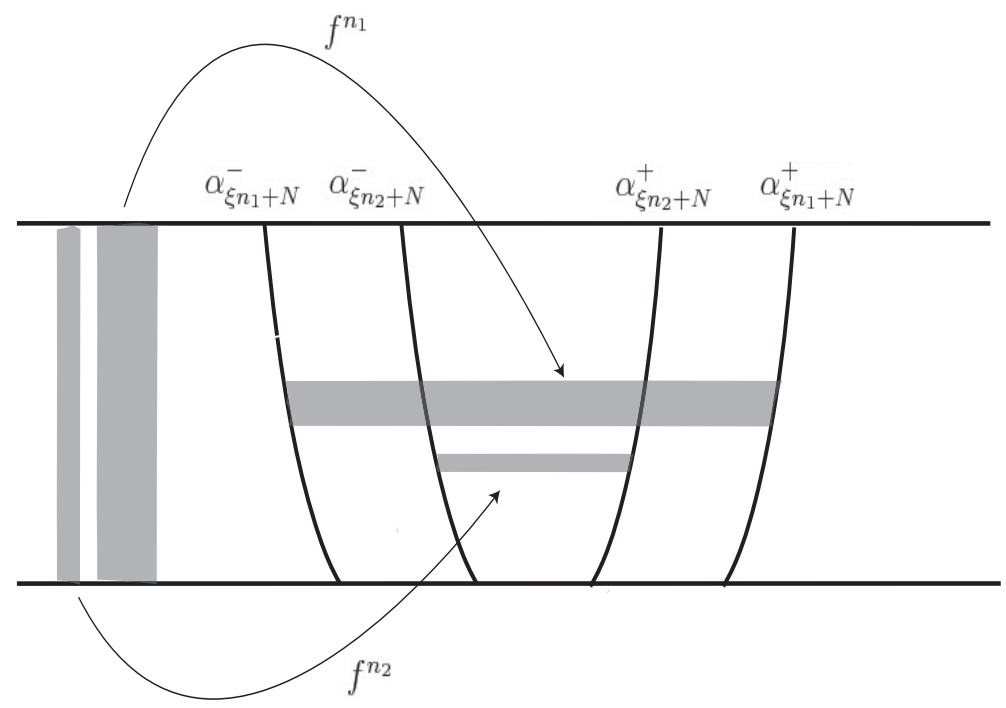

Figure 6. Schematic picture of gaps of $\mathcal{W}^{s}$. The two shaded vertical rectangles are gaps of $\mathcal{W}^{s}$ of order $n_{1}$ and $n_{2}, n_{1}<n_{2}$. Each gap of $\mathcal{W}^{s}$ of order $n \geq 0$ is mapped by $f^{n}$ to a rectangle whose stable sides are in $\alpha_{\xi n+N}^{ \pm}$and whose unstable sides are $C^{2}(b)$-curves in $W^{u}$.

connect points in $\overline{R \backslash \Theta_{n}}$ and $\Theta_{n}$ by curves in $W^{s}(P) \cap R$. Hence we obtain $f^{n+\tau(\omega)} z \notin \overline{R \backslash \Theta_{n}}$ and so $z \in \Omega_{n+\tau(\omega)}$.

For the next step of the induction, consider a gap $G$ of $\mathcal{W}^{s}$ of order $g$ and let $\gamma \subset$ $\omega \in \mathcal{Q}$ be such that $f^{\tau(\omega)} \gamma$ is a $C^{2}(b)$ curve connecting the two stable sides of $G$. Let $\omega^{\prime} \subset \gamma$ be the preimage under $f^{\tau(\omega)+g}$ of an element of the partition $\mathcal{P}\left(f^{\tau(\omega)+g} \gamma\right)$ such that $\omega^{\prime}$ contains points of $\Omega_{\infty}$. Then $f^{m+\tau(\omega)+g} \omega^{\prime} \in \tilde{\Gamma}^{u}$, where $m=\tau\left(f^{\tau(\omega)+g} \omega^{\prime}\right)$. Let $\tilde{\omega}:=f^{-m-\tau(\omega)-g}\left(f^{m+\tau(\omega)+g} \omega^{\prime} \cap \mathcal{W}^{s} \backslash\left(\alpha_{1}^{-} \cup \alpha_{1}^{+}\right)\right)$. The next sublemma justifies this construction.

Sublemma 3.12. $\tilde{\omega} \subset \Omega_{\infty}$.

Proof. By the definition of $\tau(\omega)$, we have $f^{i} \omega^{\prime} \subset \overline{R \backslash \Theta}$ for $0<i<\tau(\omega)$. We have $f^{\tau(\omega)} \omega^{\prime} \subset G$, and so by the definition of the gap $G$ of order $g, f^{j}\left(f^{\tau(\omega)} \omega^{\prime}\right) \subset \overline{R \backslash \Theta_{j}}$ for $0 \leq j \leq g-1$, and $f^{g+\tau(\omega)} \omega^{\prime} \subset \Theta_{g}$. Since $\Theta_{\tau(\omega)+j} \subset \Theta_{j}$, we in fact get $f^{j}\left(f^{\tau(\omega)} \omega^{\prime}\right) \subset \overline{R \backslash \Theta_{j+\tau(\omega)}}$ for $0 \leq j \leq g-1$. However, since $\omega^{\prime} \cap \Omega_{\infty} \neq \emptyset$ we have $f^{\tau(\omega)+g} \omega^{\prime} \subset \Theta_{g} \backslash \Theta_{g+\tau(\omega)}$, and thus $\omega^{\prime} \subset \Omega_{g+\tau(\omega)}$.

By the definition of $m$, we have $f^{n}\left(f^{\tau(\omega)+g} \omega^{\prime}\right) \subset \overline{R \backslash \Theta}$ for every $0<n<m$, and so $\tilde{\omega} \subset \omega^{\prime} \subset \Omega_{m+g+\tau(\omega)-1}$. In addition, $f^{m+g+\tau(\omega)} \tilde{\omega} \subset \mathcal{W}^{s} \subset \overline{R \backslash \Theta_{0}}$ imply $\tilde{\omega} \subset \Omega_{m+g+\tau(\omega)}$. The argument of Lemma 3.11 shows $f^{n}\left(f^{m+g+\tau(\omega)} \tilde{\omega}\right) \subset \overline{R \backslash \Theta_{n}}$ for every $n>0$, and so $\tilde{\omega} \subset \Omega_{n+m+g+\tau(\omega)}$.

By virtue of Lemma 3.10, this construction can be repeated indefinitely. We can finish the construction of $\mathcal{Q}$ by induction. Statements (a)-(c) of Lemma 3.7 are immediate consequences of the construction.

We prove statement $(\mathrm{d})$. Let $\omega \in \mathcal{Q}$. By construction, $\overline{f^{\tau(\omega)} \omega} \backslash f^{\tau(\omega)} \omega \subset \alpha_{1}^{-} \bigcup \alpha_{1}^{+}$. Hence, $\bar{\omega} \backslash \omega \subset W^{s}(P)$. By construction, if $\tau(\omega)>2$ then $\alpha_{1}^{+} \cap \bar{\omega}=\emptyset$. If $\tau(\omega)=2$ then either $\alpha_{1}^{+} \cap \bar{\omega}=\emptyset$, or else $\alpha_{1}^{+} \cap \omega \neq \emptyset$. In both cases $\alpha_{1}^{+} \notin \bar{\omega} \backslash \omega$. Hence $\bar{\omega} \backslash \omega \subset W^{s}(P) \backslash \alpha_{1}^{+}$. 
3.5. Construction of an inducing scheme from the induced map. From $(\hat{S}, \tau)$ in Sect 3.4 we now construct an inducing scheme $(S, \tau)$ as in Proposition 3.2 .

Define an induced map $F: \bigcup_{I \in \hat{S}} I \rightarrow \Lambda$ by $F z=f^{\tau(I)} z$, where $I$ is the element of $\hat{S}$ containing z. Following the terminology and notation in Definition 2.2, define the inducing domain $X$ by

$$
X=\bigcap_{n=-\infty}^{\infty} F^{-n}\left(\bigcup_{I \in \hat{S}} I\right) .
$$

By Lemma 3.13 below, $X \neq \emptyset$. Define the collection $S$ of basic elements by

$$
S=\{I \cap X: I \in \hat{S} \text { and } I \cap X \neq \emptyset\} .
$$

By definition, elements of $S$ are pairwise disjoint and $X=\bigcup_{J \in S} J$ holds. Set

$$
X^{*}=\bigcup_{J \in S} \bar{J}
$$

Define $\tau: S \rightarrow \mathbb{N}$ by $\tau(J)=\tau(I)$, where $I$ is the element of $\hat{S}$ containing $J$.

Lemma 3.13. There are families $\Gamma^{u^{\prime}} \subset \Gamma^{u}, \Gamma^{s^{\prime}} \subset \Gamma^{s}$ such that $X$ is a lattice defined by $\Gamma^{u^{\prime}}$ and $\Gamma^{s^{\prime}}$.

Proof. Write $X=X^{-} \cap X^{+}$where $X^{-}=\bigcap_{n=1}^{\infty} F^{n}\left(\bigcup_{I \in \hat{S}} I\right)$ and $X^{+}=\bigcap_{n=0}^{\infty} F^{-n}\left(\bigcup_{I \in \hat{S}} I\right)$. Since there is an element of $\hat{S}$ containing the fixed saddle $P, X^{+}$contains $P$ and so is not an empty set. By construction, $X^{+}$is written as a union of curves in $\Gamma^{s}$.

For each $n \geq 1$ let $\Gamma_{n}^{u}$ denote the defining family of $u$-curves of the lattice $F^{n}\left(\bigcup_{I \in \hat{S}} I\right)$. Then $\bigcup_{\gamma^{u} \in \Gamma_{n}^{u}} \gamma^{u}$ is a closed set, strictly decreasing in $n$. Hence $X^{+}$is the union of $u$-curves in $\bigcap_{n=1}^{\infty} \Gamma_{n}^{u}$.

We now prove (P1)-(P4) from Proposition 3.2, and then verify (A1)-(A3) from Definition 2.2. Proofs of (P5) and (P6) are deferred to Sect.3.6.

Proof of (P1). Let $p: \Lambda \rightarrow \gamma^{u}\left(\zeta_{0}\right)$ denote the holonomy map along $\gamma^{s}$-curves, i.e., $p(x)=$ $\gamma^{u}\left(\zeta_{0}\right) \cap \gamma^{s}(x)$. For subsets $A, B$ of $\Lambda$ we use the notation $A={ }_{s} B$ if $p A=p B$. The meaning of $A \subset_{s} B$ is analogous.

Let $J \in S$. By Lemma 3.13, $J$ is a lattice defined by $\Gamma^{u^{\prime}}$ and a subset of $\Gamma^{s^{\prime}}$. By construction, for each $\gamma^{u} \in \Gamma^{u \prime}, f^{\tau(J)}\left(\gamma^{u} \cap J\right) \in \Gamma^{u}$. Hence, it suffices to show

$$
f^{\tau(J)} J \subset_{s} X \text { and } f^{\tau(J)} \bar{J}={ }_{s} X^{*} .
$$

Let $I$ denote the element of $\hat{S}$ containing $J$. Then $J={ }_{s} I \backslash \bigcup_{n \geq 0} F^{-n} \Lambda_{B}$. Hence $f^{\tau(J)} J={ }_{s}$ $f^{\tau(J)} I \backslash \bigcup_{n \geq 0} F^{-n+1} \Lambda_{B}=f^{\tau(J)} I \backslash \bigcup_{n \geq 0} F^{-n} \Lambda_{B} \subset \Lambda \backslash \bigcup_{n \geq 0} F^{-n} \Lambda_{B}={ }_{s} X$. Hence the first item in (10) holds. The second one follows from $f^{\tau(J)} J={ }_{s} f^{\tau(J)} I \backslash \bigcup_{n \geq 0} F^{-n} \Lambda_{B}$.

Proofs of (P2)-(P4). (P2) follows from the backward contraction on the leaves in $\tilde{\Gamma}^{u}$ (see [39, Lemma 4.2]) and the fact that any leaf in $\Gamma^{u}$ is a $C^{1}$-limit of leaves in $\tilde{\Gamma}^{u}$. Since $f^{\tau(J)}$ is a composition of first return maps to $\Theta$, (P3) and (P4)(a) follow from the estimates in Proposition 3.5. (P4)(b) follows from Lemma 3.6(b).

Verification of (A1). Included in (P1). 
Verification of (A2). Define a coding map $h: S^{\mathbb{Z}} \rightarrow X^{*}$ by

$$
h(\underline{a}):=\overline{J_{0}} \cap\left(\bigcap_{n \geq 1} f^{-\tau\left(J_{0}\right)} \circ \cdots \circ f^{-\tau\left(J_{n-1}\right)}\left(\overline{J_{n}}\right)\right) \cap\left(\bigcap_{n \geq 1} f^{\tau\left(J_{-1}\right)} \circ \cdots \circ f^{\tau\left(J_{-n}\right)}\left(\overline{J_{-n}}\right)\right) .
$$

To verify (A2) we show that $h$ is well-defined, and induces a measurable bijection between $S^{\mathbb{Z}} \backslash h^{-1}\left(X^{*} \backslash X\right)$ and $X$.

Well-definedness. Since the set on the right-hand-side of (11) is an intersection of a nested closed sets, it is nonempty. To show that it is a singleton, for each $n \geq 1$ denote by $Q_{n}^{s}(\underline{a}) \subset \Lambda$ the rectangle spanned by the closed $s$-sublattice:

$$
Q_{n}^{s}(\underline{a}):=\overline{J_{0}} \cap\left(\bigcap_{k=1}^{n} f^{-\tau\left(J_{0}\right)} \circ \cdots \circ f^{-\tau\left(J_{k-1}\right)}\left(\overline{J_{k}}\right)\right),
$$

and define $Q_{n}^{u}(\underline{a})$ similarly:

$$
Q_{n}^{u}(\underline{a}):=\overline{J_{0}} \cap\left(\bigcap_{k=1}^{n} f^{\tau\left(J_{-1}\right)} \circ \cdots \circ f^{\tau\left(J_{-k}\right)}\left(\overline{J_{k}}\right)\right) .
$$

We have

$$
h(\underline{a}) \subset\left(\bigcap_{n \geq 1} Q_{n}^{s}(\underline{a})\right) \cap\left(\bigcap_{n \geq 1} Q_{n}^{u}(\underline{a})\right) .
$$

By $(\mathrm{P} 1),\left\{Q_{n}^{s}(\underline{a})\right\}$ is a nested sequence of nonempty closed sets. Hence, $\bigcap_{n>1} Q_{n}^{s}(\underline{a}) \neq \emptyset$. By (P3) and the fact that $\Lambda$ is closed, the boundary curves of $Q_{n}^{s}(\underline{a})$ in $\Gamma^{s}$ converge, in the $C^{0}$ topology, to a curve in $\Gamma^{s}$. Hence, $\bigcap_{n>1} Q_{n}^{s}(\underline{a}) \in \Gamma^{s}$. In the same way we have $\bigcap_{n \geq 1} Q_{n}^{u}(\underline{a}) \in \Gamma^{u}$. Since each curve in $\Gamma^{s}$ intersects each curve in $\Gamma^{u}$ exactly at one point, it follows that the set on the right-hand-side of (11) is a singleton. So, $h$ is well-defined.

Measurable bijectivity. Let $\underline{a}, \underline{a^{\prime}} \in S^{\mathbb{Z}} \backslash h^{-1}\left(X^{*} \backslash X\right)$ and suppose $h(\underline{a})=h\left(\underline{a}^{\prime}\right)=x$. We have $x \in X$, and (P1) gives $F^{n} x \in X$ for every $n \geq 1$. Since elements of $S$ are pairwise disjoint, $a_{n}=a_{n}^{\prime}$ holds for every $n \geq 0$. In addition, the next lemma implies $a_{n}=a_{n}^{\prime}$ for every $n<0$.

Lemma 3.14. For $J, J^{\prime} \in S$ distinct either $f^{\tau(J)} \bar{J} \cap f^{\tau\left(J^{\prime}\right)} \overline{J^{\prime}}=\emptyset$ or $f^{\tau(J)} \bar{J} \cap f^{\tau\left(J^{\prime}\right)} \overline{J^{\prime}} \subset X^{*} \backslash X$.

Proof. It is obvious that if $\tau(J)=\tau\left(J^{\prime}\right)$ then $f^{\tau(J)} \bar{J} \cap f^{\tau\left(J^{\prime}\right)} \overline{J^{\prime}}=\emptyset$. Suppose $\tau(J)>\tau\left(J^{\prime}\right)$ and $f^{\tau(J)} \bar{J} \cap f^{\tau\left(J^{\prime}\right)} \overline{J^{\prime}} \neq \emptyset$. Then $f^{\tau(J)-\tau\left(J^{\prime}\right)} \bar{J} \cap \overline{J^{\prime}} \neq \emptyset$ and since $\tau(J)$ is the first entry time of $J$ to $\Lambda$ and $J^{\prime} \subset \Lambda$, then $f^{\tau(J)-\tau\left(J^{\prime}\right)} \bar{J} \cap \overline{J^{\prime}} \subset \overline{J^{\prime}} \backslash J^{\prime}$. The intersection thus belongs to $X^{*} \backslash X$.

Consequently, $\tilde{h}:=\left.h\right|_{S^{\mathbb{Z}} \backslash h^{-1}\left(X^{*} \backslash X\right)}$ is injective. Since $h$ is onto $X, \tilde{h}$ is onto $X$ as well. From the uniform hyperbolicity of $F$ and the fact that the cylinder sets form a base of the topology in $S^{\mathbb{Z}}, \tilde{h}$ is continuous and maps open sets to Borel sets. By [39, Claim 3.3], $\tilde{h}^{-1}$ is measurable.

Verification of (A3). From Corollary $\left[3.7(\mathrm{~d})\right.$ and the construction, $\bar{J} \backslash J \subset\left(W^{s}(P) \backslash\{P\}\right) \cup$ $\bigcup_{n \geq 0} F^{-n} \Lambda_{B}$ holds for each $J \in S$. By the next lemma, no $f$-invariant probability measure gives positive weight to $X^{*} \backslash X$.

Lemma 3.15. $\bigcup_{n>0} F^{-n} \Lambda_{B}$ is a s-sublattice of $\Lambda$. In addition, for any $\mu \in \mathcal{M}(f)$ one has that $\mu\left(\bigcup_{n \geq 0} F^{-n} \Lambda_{B}\right)=0$. 


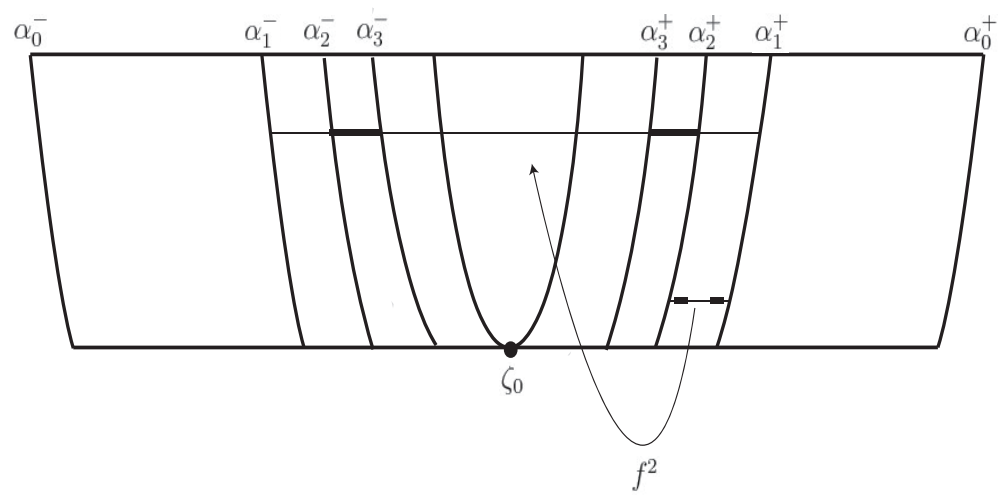

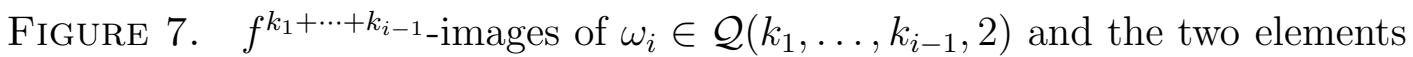
of $\mathcal{Q}\left(\omega_{i}, 3\right)$, and their $f^{2}$-images.

Proof. As in the previous remark, $\Lambda_{B}$ is an $s$-sublattice of $\Lambda$. Since $F$ maps each $\gamma^{s}$-curve into a subset of an $\gamma^{s}$-curve, $F^{-n} \Lambda_{B}$ is an $s$-sublattice of $\Lambda$ for every $n>0$. Hence the first statement holds. As for the second one, since $f^{-n} \Lambda_{B}(n=0,1, \ldots)$ are pairwise disjoint, $\mu\left(\Lambda_{B}\right)=0$. Hence $\mu\left(f^{-n} \Lambda_{B}\right)=0$ and so $\mu\left(\bigcup_{n \geq 0} F^{-n} \Lambda_{B}\right)=0$.

If there is a $\sigma$-invariant probability measure $\nu$ which gives positive weight to $h^{-1}\left(X^{*} \backslash X\right)$, then $h_{*} \nu$ gives positive weight to $X^{*} \backslash X$, and so does $\mathcal{L}\left(h_{*} \nu\right) \in \mathcal{M}(f)$. This is a contradiction.

3.6. Comments on proofs of (P5) (P6). To prove (P5) and (P6) we use the fact that the inducing scheme $(S, \tau)$ is built over the first entry time to $\Lambda$. To prove (P6) it suffices to show that any ergodic measure with entropy $\geq 2 \varepsilon$ gives positive weight to $X$. This amounts to showing that the first entry time to $\Lambda$ is finite, with positive probability for these measures.

The rest of this section is organized as follows. Sect 3.7 and Sect 3.9 are devoted to estimates of the Hausdorff dimension of sets of points not returning to $\Lambda$. (P5) is proved in Sect 3.8 with the estimate obtained in Sect 3.7. (P6) is proved in Sect.3.10.

3.7. Hausdorff dimension of the set of points in $\Lambda$ not returning to $\Lambda$. By Lemma 3.6, the tangent directions of the curves in $\Gamma^{s}$ are Lipschitz continuous, and so the holonomy map between two curves in $\tilde{\Gamma}^{u}$ along $\gamma^{s}$-curves is Lipschitz continuous. Hence, for any $s$-sublattice $\Lambda^{\prime}$ of $\Lambda$ the Hausdorff dimension of $\gamma^{u} \cap \Lambda^{\prime}$, denoted by $d^{u}\left(\Lambda^{\prime}\right)$, is independent of the choice of $\gamma^{u} \in \tilde{\Gamma}^{u}$.

Lemma 3.16. $d^{u}\left(\Lambda_{B}\right) \leq \varepsilon$.

Proof. Since $d^{u}\left(\Lambda_{B}\right)=\operatorname{dim}_{H}^{u}\left(\Omega_{\infty} \cap B\right)$ it suffices to show $\operatorname{dim}_{H}^{u}\left(\Omega_{\infty} \cap B\right) \leq \varepsilon$. Given $g>1$ and a $g$-string $\left(k_{1}, \ldots, k_{g}\right)$ of positive integers, we define collections $\mathcal{Q}\left(k_{1}, k_{2}, \ldots, k_{i}\right)(i=$ $1,2, \ldots, g)$ of pairwise disjoint curves in $\gamma^{u}\left(\zeta_{0}\right)$ inductively as follows. Let

$$
\mathcal{Q}\left(k_{1}\right)=\left\{\omega_{1} \subset \gamma^{u}\left(\zeta_{0}\right): f^{k_{1}} \omega_{1} \in \tilde{\Gamma}^{u}\right\} .
$$

Given $\mathcal{Q}\left(k_{1}, \ldots, k_{i}\right)$, for each $\omega_{i} \in \mathcal{Q}\left(k_{1}, \ldots, k_{i}\right)$ let

$$
\mathcal{Q}\left(\omega_{i}, k_{i+1}\right)=\left\{\omega_{i+1} \subset \omega_{i}: f^{k_{1}+\cdots+k_{i}+k_{i+1}} \omega_{i+1} \in \tilde{\Gamma}^{u}\right\},
$$


and define inductively

$$
\mathcal{Q}\left(k_{1}, \ldots, k_{i+1}\right)=\bigcup_{\omega_{i} \in \mathcal{Q}\left(k_{1}, \ldots, k_{i}\right)} \mathcal{Q}\left(\omega_{i}, k_{i+1}\right)
$$

(See FIGURE 7).

For $n>0$ let

$$
\mathcal{Q}_{n}\left(k_{1}, \ldots, k_{i}\right)=\left\{\omega_{i} \in \mathcal{Q}\left(k_{1}, \ldots, k_{i}\right): \sup \left\{\tau(z): z \in \omega_{i}\right\} \geq n\right\},
$$

and

$$
\mathcal{Q}_{n}\left(\omega_{i}, k_{i+1}\right)=\left\{\omega_{i+1} \in \mathcal{Q}\left(\omega_{i}, k_{i+1}\right): \sup \left\{\tau(z): z \in \omega_{i+1}\right\} \geq n\right\} .
$$

Let $\omega_{0}=\gamma^{u}\left(\zeta_{0}\right)$ and $\mathcal{Q}_{n}\left(\omega_{0}, k_{1}\right)=\mathcal{Q}_{n}\left(k_{1}\right)$.

Sublemma 3.17. If $N>2(1+\xi)$, then for every $n>6 N$ and for any $z \in \Omega_{\infty}$ with $\tau(z) \geq n$ there exist an integer $1 \leq s \leq n / N$, and for each $i=1, \ldots, s$ an integer $k_{i} \geq N$ and a curve $\omega_{i} \in \mathcal{Q}_{n}\left(k_{1}, \ldots, k_{i}\right)$ such that:

(a) $\frac{n}{3 \xi} \leq k_{1}+\cdots+k_{s} \leq n$;

(b) $z \in \omega_{s} \subset \cdots \subset \omega_{1}$;

(c) $\ell\left(\omega_{s}\right) \leq C \sigma_{1}^{-\left(k_{1}+\cdots+k_{s}\right)}$;

(d) for each $i=0, \ldots, s-1$, \# $\mathcal{Q}_{n}\left(\omega_{i}, k_{i+1}\right)<2^{\frac{k_{i+1}}{\xi}}$.

Proof. For $z \in \Omega_{\infty}$ define a sequence $0=: t_{0}<t_{1}<\cdots$ of return times to $\Theta$ inductively as follows: given $t_{i}$ such that $f^{t_{i}} z$ is in the gap of $\mathcal{W}^{s}$ of order $g_{i}$, define

$$
t_{i+1}=\min \left\{t>t_{i}+g_{i}: f^{t} z \in \Theta\right\} .
$$

Note that $\left\{t_{i}\right\}$ are not the only return times of the orbit of $z$ to $\Theta$. Since $f^{t_{i}} z$ is in the gap of order $g_{i}$, one has $t_{i+1} \geq t_{i}+g_{i}+\xi g_{i}+N$ and $g_{i} \geq 0$ we have

$$
t_{i+1}-t_{i} \geq N
$$

Define $s:=\max \left\{i: t_{i}<n\right\}+1$. (12) gives $s \leq n / N$.

Claim 3.18. $t_{s-1}+g_{s-1} \geq \frac{n}{3 \xi}$.

Proof. Suppose the contrary. Then $\xi\left(t_{s-1}+g_{s-1}\right)+N<[n / 2]$. Since $f^{t_{s-1}} z$ is in a gap of

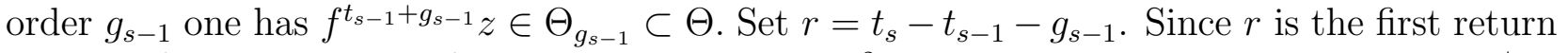
time of $f^{t_{s-1}+g_{s-1}} z$ to $\Theta, f^{t_{s-1}+g_{s-1}} z$ belongs to a $C^{2}(b)$-curve connecting either $\alpha_{r}^{+}$and $\alpha_{r+1}^{+}$, or $\alpha_{r}^{-}$and $\alpha_{r+1}^{-}$. Since $f^{t_{s-1}+g_{s-1}} z \notin \Theta_{t_{s-1}+g_{s-1}}$ by the assumption $z \in \Omega_{\infty}$ (see (9)), one has $r<\xi\left(t_{s-1}+g_{s-1}\right)+N<[n / 2]$ and $t_{s}<\frac{n}{3 \xi}+[n / 2]<n$, a contradiction to $t_{s} \geq n$.

For each $i=1, \ldots, s$, set $k_{i}=t_{i}-t_{i-1}$ and let $\omega_{i}$ denote the element of $\mathcal{Q}_{n}\left(k_{1}, \ldots, k_{i}\right)$ containing $z$. (3.18) and the definition of $s$ give $t_{s-1}+g_{s-1}<t_{s} \leq n$. Since $k_{1}+\cdots+k_{s}=t_{s}$, (a) holds. (b) is straightforward. (c) follows from Lemma 3.5.

In order to prove $(\mathrm{d})$, observe that for each $\omega \in \mathcal{Q}_{n}\left(\omega_{i}, k_{i+1}\right)$ there exists a gap $G$ of $\mathcal{W}^{s}$ such that $f^{k_{1}+\cdots+k_{i}} \omega \subset G$. In addition, for any gap $G$ of $\mathcal{W}^{s}$ we have

$$
\#\left\{\omega \in \mathcal{Q}_{n}\left(\omega_{i}, k_{i+1}\right): f^{k_{1}+\cdots+k_{i}} \omega \subset G\right\}=0 \text { or }=2,
$$

which is because gaps are not folded up to their order, and $f^{j} G \cap \Theta=\emptyset$ for $g_{i}<j<t_{i+1}$ by the definition of $t_{i+1}$. There is therefore at most one fold at time $t_{i}+g_{i}$. 
Let $g_{0}$ denote the maximal order of the gap of $\mathcal{W}^{s}$ which contains $f^{k_{1}+\cdots+k_{i}}$-images of elements of $\mathcal{Q}_{n}\left(\omega_{i}, k_{i+1}\right)$. Then $g_{0}+\xi g_{0}+N \leq k_{i+1}$ holds. From (13) and the fact that the number of gaps of order $g$ is $\leq 2^{g}$ we obtain $\# \mathcal{Q}_{n}\left(\omega_{i}, k_{i+1}\right) \leq 2 \sum_{i=1}^{g_{0}} 2^{i}<2^{\frac{k_{i+1}-N}{1+\xi}+2}<2^{\frac{k_{i+1}}{\xi}}$. The last inequality holds provided $N>2(1+\xi)$.

Returning to the proof of Lemma 3.16, we have

$$
\Omega_{\infty} \cap B \subset\left\{z \in \Omega_{\infty}: \tau(z) \geq n\right\} \subset \bigcup_{s=1}^{\left[\frac{n}{N}\right]} \bigcup_{l=\left[\frac{n}{3 \xi}\right]}^{n} \bigcup_{k_{1}+\cdots+k_{s}=l} \bigcup_{\omega_{s} \in \mathcal{Q}_{n}\left(k_{1}, \ldots, k_{s}\right)} \omega_{s} .
$$

By Sublemma 3.17(a)(c), the lengths of the curves $\omega_{s}$ in the union of the right-hand-side are

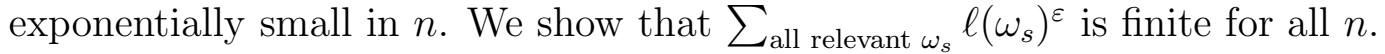

Observe that

$$
\sum_{\omega_{i+1} \in \mathcal{Q}_{n}\left(k_{1}, \ldots, k_{i+1}\right)} \ell\left(\omega_{i+1}\right)^{\varepsilon}=\sum_{\omega_{i} \in \mathcal{Q}_{n}\left(k_{1}, \ldots, k_{i}\right)} \ell\left(\omega_{i}\right)^{\varepsilon} \sum_{\omega_{i+1} \in \mathcal{Q}_{n}\left(\omega_{i}, k_{i+1}\right)} \frac{\ell\left(\omega_{i+1}\right)^{\varepsilon}}{\ell\left(\omega_{i}\right)^{\varepsilon}} .
$$

On the second sum of the fractions, since $\ell\left(f^{k_{1}+\cdots+k_{i+1}} \omega_{i+1}\right)<2$ for each $\omega_{i+1} \in \mathcal{Q}_{n}\left(\omega_{i}, k_{i+1}\right)$ and $\left\|D_{x} f^{k_{i+1}} \mid E^{u}\right\| \geq \sigma_{1}^{k_{i+1}}$ for all $x \in f^{k_{1}+\cdots+k_{i}} \omega_{i+1}$, we have $\ell\left(f^{k_{1}+\cdots+k_{i}} \omega_{i+1}\right) \leq(1 / 2) \sigma_{1}^{-k_{i+1}}$. From this and the bounded distortion in Lemma 3.5.

$$
\frac{\ell\left(\omega_{i+1}\right)}{\ell\left(\omega_{i}\right)} \leq C \cdot \frac{\ell\left(f^{k_{1}+\cdots+k_{i}} \omega_{i+1}\right)}{\ell\left(f^{k_{1}+\cdots+k_{i}} \omega_{i}\right)} \leq C \sigma_{1}^{-k_{i+1}} .
$$

Using (15) and Sublemma $3.17(\mathrm{~d})$,

$$
\sum_{\omega_{i+1} \in \mathcal{Q}_{n}\left(\omega_{i}, k_{i+1}\right)} \frac{\ell\left(\omega_{i+1}\right)^{\varepsilon}}{\ell\left(\omega_{i}\right)^{\varepsilon}} \leq \# \mathcal{Q}_{n}\left(\omega_{i}, k_{i+1}\right) C^{\varepsilon} \sigma_{1}^{-\varepsilon k_{i+1}} \leq C^{\varepsilon} \sigma_{1}^{-\frac{\varepsilon}{2} k_{i+1}} .
$$

Plugging this into the right-hand-side of (14) we get

$$
\sum_{\omega_{i} \in \mathcal{Q}_{n}\left(k_{1}, \ldots, k_{i+1}\right)} \ell\left(\omega_{i+1}\right)^{\varepsilon} \leq C^{\varepsilon} \sigma_{1}^{-\varepsilon k_{i+1}} \sum_{\omega_{i} \in \mathcal{Q}_{n}\left(k_{1}, \ldots, k_{i}\right)} \ell\left(\omega_{i}\right)^{\varepsilon} .
$$

The same arguments as above applied to any $\omega_{1} \in \mathcal{Q}_{n}\left(k_{1}\right)$ yield

$$
\sum_{\omega_{1} \in \mathcal{Q}_{n}\left(k_{1}\right)} \ell\left(\omega_{1}\right)^{\varepsilon} \leq C^{\varepsilon} \# \mathcal{Q}_{n}\left(k_{1}\right) \sigma_{1}^{-\varepsilon k_{1}} \leq C^{\varepsilon} \sigma_{1}^{-\frac{\varepsilon}{2} k_{1}} .
$$

Using (16) inductively and (17) yields

$$
\sum_{\omega_{s} \in \mathcal{Q}_{n}\left(k_{1}, \ldots, k_{s}\right)} \ell\left(\omega_{s}\right)^{\varepsilon} \leq C^{\varepsilon s} \sigma_{1}^{-\frac{\varepsilon}{2}\left(k_{1}+\cdots+k_{s}\right)} .
$$

Hence

$$
\sum_{l=\left[\frac{n}{3 \xi}\right]}^{n} \sum_{k_{1}+\cdots+k_{s}=l} \sum_{\omega_{s} \in \mathcal{Q}_{n}\left(k_{1}, \ldots, k_{s}\right)} \ell\left(\omega_{s}\right)^{\varepsilon} \leq C^{\varepsilon s} \sum_{l=\left[\frac{n}{3 \xi}\right]}^{n} \sigma_{1}^{-\frac{\varepsilon l}{2}} \#\left\{\left(k_{1}, \ldots, k_{s}\right): \sum_{i=1}^{s} k_{i}=l\right\} .
$$

To estimate the right-hand side we use the following from Stirling's formula for factorials (see e.g. [15]): for sufficiently small $\chi>0$ there exist $c(\chi)>0$ with $c(\chi) \rightarrow 0$ as $\chi \rightarrow 0$ such that 
for any two positive integers $p, q$ with $q / p \leq \chi$ one has $(\underset{q}{p+q}) \leq e^{c(\chi) p}$. Since $k_{i} \geq N$ and $s / l \leq 1 / N$, one has that $e^{c(1 / N)} \leq \sigma_{1}^{\frac{\varepsilon}{3}}$ for sufficiently large $N$ and thus

$$
\#\left\{\left(k_{1}, \ldots, k_{s}\right): \sum_{i=1}^{s} k_{i}=l\right\} \leq\left(\begin{array}{c}
l+s \\
s
\end{array}\right) \leq \sigma_{1}^{\frac{\varepsilon}{3} l}
$$

Then

$$
\sum_{l=\left[\frac{n}{3 \xi}\right]}^{n} \sum_{k_{1}+\cdots+k_{s}=l} \sum_{\omega_{s} \in \mathcal{Q}_{n}\left(k_{1}, \ldots, k_{s}\right)} \ell\left(\omega_{s}\right)^{\varepsilon} \leq C^{\varepsilon s} \sum_{l=\left[\frac{n}{3 \xi}\right]}^{n} \sigma_{1}^{-\frac{\varepsilon}{6} l}
$$

Hence

$$
\sum_{s=1}^{\left[\frac{n}{N}\right]} \sum_{l=\left[\frac{n}{3 \xi}\right]}^{n} \sum_{k_{1}+\cdots+k_{s}=l} \sum_{\omega_{s} \in \mathcal{Q}_{n}\left(k_{1}, \ldots, k_{s}\right)} \ell\left(\omega_{s}\right)^{\varepsilon} \leq \frac{C^{\frac{\varepsilon n}{N}}}{C^{\varepsilon}-1} \sum_{l=\left[\frac{n}{3 \xi}\right]}^{n} \sigma_{1}^{-\frac{\varepsilon}{6} l} .
$$

One can choose $N \geq N_{0}$ large enough at the expense of reducing $b>0$ so that the expression on the right-hand-side decays exponentially with $n$. Consequently the Hausdorff $\varepsilon$-measure of $\Omega_{\infty} \cap B$ is zero.

3.8. Proof of (P5). We are in position to prove (P5) in Proposition 3.2.

Proof of (P5). For each $J \in S$ with $\tau(J)=n$, let $\omega_{J}$ denote the unstable side of the rectangle $Q_{J}$ spanned by $J$ which is contained in $\gamma^{u}\left(\zeta_{0}\right)$. Observe that there exists $1 \leq s \leq n / N$ and an $s$-string $\left(k_{1}, \ldots, k_{s}\right)$ of positive integers such that $k_{1}+\cdots+k_{s}=n$ and $\omega_{J} \in \overline{\mathcal{Q}}_{n}\left(k_{1}, \ldots, k_{s}\right)$. For two distinct $J_{1}, J_{2} \in S$ with $\tau\left(J_{1}\right)=\tau\left(J_{2}\right)=n$, one has $\omega_{J_{1}} \cap \omega_{J_{2}}=\emptyset$. Therefore,

$$
S(n) \leq \sum_{s=1}^{\left[\frac{n}{N}\right]} \sum_{k_{1}+\cdots+k_{s}=n} \# \mathcal{Q}_{n}\left(k_{1}, \ldots, k_{s}\right) .
$$

Using Sublemma $3.17(\mathrm{~d})$ repeatedly and $\# \mathcal{Q}_{n}\left(k_{1}\right) \leq 2$,

$$
\begin{aligned}
\# \mathcal{Q}_{n}\left(k_{1}, \ldots, k_{s}\right) & =\sum_{\omega \in \mathcal{Q}_{n}\left(k_{1}, \ldots, k_{s-1}\right)} \# \mathcal{Q}\left(\omega, k_{s}\right) \\
& \leq \# \mathcal{Q}_{n}\left(k_{1}, \ldots, k_{s-1}\right) \max _{\omega \in \mathcal{Q}_{n}\left(k_{1}, \ldots, k_{s-1}\right)} \# \mathcal{Q}\left(\omega, k_{s}\right) \\
& \leq \# \mathcal{Q}_{n}\left(k_{1}, \ldots, k_{s-1}\right) \cdot 2^{\frac{k_{s}}{\xi}} \\
& \leq \cdots \\
& \leq \# \mathcal{Q}_{n}\left(k_{1}\right) \cdot 2^{\frac{1}{\xi}\left(k_{2}+\cdots+k_{s}\right)} \\
& \leq 2^{\frac{1}{\xi}\left(k_{1}+\cdots+k_{s}\right)+2}
\end{aligned}
$$

The above counting argument shows that the number of all feasible combinations $\left(k_{1}, \ldots, k_{s}\right)$ is $\leq \sigma_{1}^{\frac{\varepsilon}{3} n}$. Hence we obtain $S(n) \leq(n / N) \sigma_{1}^{\frac{\varepsilon}{3} n} 2^{\frac{n}{\xi}}$, and thus

$$
\varlimsup_{n \rightarrow \infty} \frac{1}{n} \log S(n) \leq \frac{\varepsilon}{3} \log \sigma_{1}+\frac{1}{\xi} \log 2 \leq \varepsilon .
$$

The last inequality follows from the definition of $\xi$ in (8) . 
3.9. Dimension of the set of points in open sets in $W^{u}$ not returning to $\Lambda$. Using Lemma 3.16 we estimate the Hausdorff dimension of the set of points in open sets in $W^{u}$ which do not return to $\Lambda$ under any positive iteration.

Lemma 3.19. For any open set $\gamma$ in $W^{u}$, $\operatorname{dim}_{H}^{u}(\gamma \cap B) \leq \varepsilon$.

Proof. To estimate the Hausdorff dimension of the set $\gamma \cap B$ we decompose it into the union of points belonging to $\Lambda$, points of the complement whose orbit eventually belongs to $\Lambda$ and points of the complement which never enter $\Lambda$. The proof of Lemma 3.16 allows us to obtain an upper estimate of the Hausdorff dimension of this subset. For the complement of this subset we need a different computation.

Sublemma 3.20. For each $\gamma^{u} \in \tilde{\Gamma}^{u}$ we have $\operatorname{dim}_{H}^{u}\left(\gamma^{u} \cap B\right) \leq \varepsilon$.

Proof. Let $z \in \gamma^{u} \cap \Omega$.

We call $l>0$ a close return time of $z$ if $l=\min \left\{i>0: f^{i} z \in \Theta_{i}\right\}$. Points in $\Lambda$ have no close returns. Let $l_{1}, l_{2}, \ldots$ be defined inductively as follows: $l_{1}$ is the close return time of $z$; given $l_{1}, \ldots, l_{k-1}$, let $l_{k}$ be the close return time of $f^{l_{1}+\cdots+l_{k-1}} z$. Obviously $l_{k} \geq \xi l_{k-1}+N$ and $l_{1} \geq 1$, and so

$$
l_{k} \geq \xi^{k-1}
$$

Let $\Xi_{k}$ denote the set of $z \in \gamma^{u}$ for which $l_{1}, \ldots, l_{k}$ are defined in this way. Set $\Xi_{\infty}:=\bigcap_{k \geq 1} \Xi_{k}$. Since $\gamma^{u} \backslash \Xi_{1}=\gamma^{u} \cap \Lambda$, one has that

$$
\gamma^{u} \cap B=\left(\gamma^{u} \cap \Lambda_{B}\right) \cup\left(\bigcup_{k \geq 1} \Xi_{k} \backslash \Xi_{k+1} \cap B\right) \cup\left(\Xi_{\infty} \cap B\right),
$$

and so

$$
\operatorname{dim}_{H}^{u}\left(\gamma^{u} \cap B\right) \leq \max \left\{\operatorname{dim}_{H}^{u}\left(\Lambda_{B} \cap \gamma^{u}\right), \sup _{k \geq 1} \operatorname{dim}_{H}^{u}\left(\left(\Xi_{k} \backslash \Xi_{k+1}\right) \cap B\right), \operatorname{dim}_{H}^{u}\left(\Xi_{\infty} \cap B\right)\right\} .
$$

By Lemma 3.16 $\operatorname{dim}_{H}^{u}\left(\gamma^{u} \cap \Lambda_{B}\right)=d^{u}\left(\Lambda_{B}\right) \leq \varepsilon$. In addition, for each $k \geq 1$ the set $\Xi_{k} \backslash \Xi_{k+1}$ can be decomposed into a countable collection of connected components $\left\{z \in \gamma^{u}: f^{m+n} z \notin\right.$ $\left.\Theta_{n} \forall n \geq 0\right\} \cap B$, for some $m \geq 0$. In the same way as in the proof of Lemma 3.7, one can show that $\operatorname{dim}_{H}^{u}\left(\left(\Xi_{k} \backslash \Xi_{k+1}\right) \cap B\right) \leq \varepsilon$ and

$$
\operatorname{dim}_{H}^{u}\left(\gamma^{u} \cap B\right) \leq \max \left\{\varepsilon, \operatorname{dim}_{H}^{u}\left(\Xi_{\infty}\right)\right\} .
$$

To show $\operatorname{dim}_{H}^{u}\left(\Xi_{\infty}\right) \leq \varepsilon$, let $\mathcal{U}_{k}$ denote the collection of connected components of $\Xi_{k}$. For each $u_{k} \in \mathcal{U}_{k}$ there exist a sequence $l_{1}<\cdots<l_{k}$ of positive integers and a nested sequence $u_{1} \supset \cdots \supset u_{k}$ of curves such that for each $i=1, \ldots, k, f^{l_{1}+\cdots+l_{i}} u_{i}$ is a $C^{2}(b)$-curve connecting the two stable sides of $\Theta_{l_{i}}$. For $u_{k-1} \in \mathcal{U}_{k-1}$ and $l_{k}>0$ let

$$
\mathcal{R}\left(u_{k-1}, l_{k}\right)=\left\{u_{k} \in \mathcal{U}_{k}: l_{k} \text { is a close return time of points in } f^{l_{1}+\cdots+l_{k-1}} u_{k}\right\} .
$$

By definition,

$$
\Xi_{k}=\bigcup_{u_{k-1} \in \mathcal{U}_{k-1}} \bigcup_{l_{k}} \bigcup_{u_{k} \in \mathcal{R}\left(u_{k-1}, l_{k}\right)} u_{k}
$$


where the second union runs over all possible $l_{k}$. For each $u_{k} \in \mathcal{R}\left(u_{k-1}, l_{k}\right)$, let $\widehat{u}_{k}$ denote the curve in $u_{k-1}$ containing $u_{k}$ such that $f^{l_{1}+\cdots+l_{k}} \widehat{u}_{k} \in \tilde{\Gamma}^{u}$. Since the distortion of $f^{l_{1}+\cdots+l_{k}} \mid \widehat{u}_{k}$ is uniformly bounded by Lemma 3.5, we have

$$
\frac{\ell\left(u_{k}\right)}{\ell\left(u_{k-1}\right)} \leq \frac{\ell\left(u_{k}\right)}{\ell\left(\widehat{u}_{k}\right)} \leq C \frac{\ell\left(f^{l_{1}+\cdots+l_{k}} u_{k}\right)}{\ell\left(f^{l_{1}+\cdots+l_{k}} \widehat{u}_{k}\right)} \leq C \sigma_{1}^{-\xi l_{k}}
$$

Set $\eta:=2 \sigma_{1}^{-\varepsilon \xi}$. By the definition of $\xi$ in (18) $), \eta<1$. Using $\# \mathcal{R}\left(u_{k-1}, l_{k}\right) \leq 2^{l_{k}}$ and (18) we get

$$
\sum_{l_{k}} \sum_{u_{k} \in \mathcal{R}\left(u_{k-1}, l_{k}\right)} \frac{\ell\left(u_{k}\right)^{\varepsilon}}{\ell\left(u_{k-1}\right)^{\varepsilon}} \leq C^{\varepsilon} \sum_{l_{k} \geq \xi^{k-1}} 2^{l_{k}} \sigma_{1}^{-\varepsilon \xi l_{k}} \leq C^{\varepsilon} \eta^{\xi^{k-1}} \text {. }
$$

Hence

$$
\sum_{u_{k} \in \mathcal{U}_{k}} \ell\left(u_{k}\right)^{\varepsilon}=\sum_{u_{k-1} \in \mathcal{U}_{k-1}} \ell\left(u_{k-1}\right)^{\varepsilon}\left(\sum_{l_{k}} \sum_{u_{k} \in \mathcal{R}\left(u_{k-1}, l_{k}\right)} \frac{\ell\left(u_{k}\right)^{\varepsilon}}{\ell\left(u_{k-1}\right)^{\varepsilon}}\right) \leq C^{\varepsilon} \eta^{\xi^{k-1}} \sum_{u_{k-1} \in \mathcal{U}_{k-1}} \ell\left(u_{k-1}\right)^{\varepsilon} .
$$

Using this recursively yields

$$
\sum_{u_{k} \in \mathcal{U}_{k}} \ell\left(u_{k}\right)^{\varepsilon} \leq C^{\varepsilon(k-1)} \eta^{\sum_{i=1}^{k-1} \xi^{i-1}} \sum_{u_{1} \in \mathcal{U}_{1}} \ell\left(u_{1}\right)^{\varepsilon} .
$$

The right-hand-side goes to 0 as $k \rightarrow \infty$, and thus the Hausdorff $\varepsilon$-measure of $\Xi_{\infty}$ is 0 .

A successive use of Lemma 3.5 implies that any point in $\gamma \cap \Omega$ is contained in a curve in $W^{u}$ which is mapped by some forward iterates to a curve in $\tilde{\Gamma}^{u}$. Hence, the countable stability of Hausdorff dimension and Sublemma 3.20 complete the proof of Lemma 3.19.

3.10. Proof of (P6). We recall a few general results on stable and unstable manifolds of nonuniformly hyperbolic systems from [28, 35]. Since any $\mu \in \mathcal{M}^{e}(f)$ has one positive and one negative Lyapunov exponent [12, these general results hold for our system.

For any $\mu \in \mathcal{M}^{e}(f)$ there exist Borel subsets $\Gamma_{1} \subset \Gamma_{2} \subset \cdots \subset \Omega$ such that $\operatorname{supp}(\mu)=$ $\Gamma_{\infty}:=\bigcup \Gamma_{n}$ and sequences of positive numbers $\delta_{n} \gg \epsilon_{n}$, possibly $\rightarrow 0$ as $n \rightarrow \infty$, such that, for $x \in \Gamma_{n}$ :

(N1) the unstable manifold $W^{u}(x)$ of $x$ (see (3) ) is an injectively immersed $C^{2}$ submanifold with $T_{x} W^{u}(x)=E^{u}(x)$. An analogous statement holds for the stable manifold $W^{s}(x)$.

Let $B_{\delta}^{u}(x)$ (resp. $B_{\delta}^{s}(x)$ ) denote the ball of radius $\delta$ centered at the origin of $T_{x} \mathbb{R}^{2}$ in $E^{u}(x)$ (resp. $E^{s}(x)$ ) and $B_{\delta}(x):=B_{\delta}^{u}(x) \times B_{\delta}^{s}(x)$. Let $\Gamma_{n}(x):=\left\{y \in \Gamma_{n}:|x-y|<\epsilon_{n}\right\}$ and for $y \in \Gamma_{n}(x)$, let $W_{x}^{u}(y)$ denote the connected component of $\exp _{x}^{-1}\left(W^{u}(y) \cap \exp _{x}\left(B_{\delta_{n}}(x)\right)\right)$ that contains $\exp _{x}^{-1} y$.

(N2) For all $y \in \Gamma_{n}(x), W_{x}^{u}(y)$ is the graph of a function $\varphi: B_{\delta_{n}}^{u}(x) \rightarrow B_{\delta_{n}}^{s}(x)$ with $\|D \varphi\| \leq$ $\frac{1}{100}$, for a conveniently chosen metric. An analogous statement holds for $W_{x}^{s}(y)$.

(N3) For $z \in \bigcup_{y \in \Gamma_{n}(x)} W_{x}^{s}(y)$, let $\mathcal{F}^{s}(z)$ denote the element of $\left\{W_{x}^{s}(y)\right\}_{y \in \Gamma_{n}(x)}$ which contains $z$. Then $z \mapsto T_{z} \mathcal{F}^{s}(z)$ is Lipschitz continuous.

(N4) The holonomy map $\pi: \Sigma_{1} \cap \bigcup_{y \in \Gamma_{n}(x)} W_{x}^{s}(y) \rightarrow \Sigma_{2}$ defined by $\pi(y)=W_{x}^{s}(y) \cap \Sigma_{2}$ for any graph $\Sigma_{i}(i=1,2)$ of a $C^{1}$ function $\psi_{i}: B_{\delta_{n}}^{u}(x) \rightarrow B_{\delta_{n}}^{s}(x)$ with $\left\|D \psi_{i}\right\| \leq \frac{1}{99}$ is bi-Lipschitz continuous. In particular, it preserves Hausdorff dimension. 
Remark 3.5. Since $\operatorname{dim} E^{u}=1$ the constant $\alpha$ in the bunching condition [19, (19.1.1)] can be taken to be 1. Then (N3) follows from a slight modification of the proof of [19, Theorem 19.1.6]. (N4) follows from (N3) and the fact that $\operatorname{dim} E^{s}=1$.

Let $x \in \Gamma_{\infty}$. For each $n>0$ consider a countable covering $\left\{\Gamma_{n}\left(z_{i}\right)\right\}_{i}$ of $\Gamma_{n} \cap W^{u}(x)$ such that $\bigcup_{i} W_{\mathrm{loc}}^{u}\left(z_{i}\right)=\Gamma_{n} \cap W^{u}(x)$, where $W_{\mathrm{loc}}^{u}\left(z_{i}\right):=\exp _{z_{i}} W_{z_{i}}^{u}\left(z_{i}\right)$. Let $B_{i}=W_{\mathrm{loc}}^{u}\left(z_{i}\right) \cap B$.

Lemma 3.21. $\operatorname{dim}_{H}^{u}\left(B_{i}\right) \leq \varepsilon$.

Proof. By Katok's closing lemma [19, Theorem S.4.13], there exists a periodic saddle $p_{i} \in$ $\Gamma_{n}\left(z_{i}\right)$ such that $W_{z_{i}}^{u}\left(p_{i}\right)$ is the graph of a function $\varphi: B_{\delta_{n}}^{u}\left(z_{i}\right) \rightarrow B_{\delta_{n}}^{s}\left(z_{i}\right)$ with $\|D \varphi\| \leq$ $\frac{1}{100}$. Since $W^{s}\left(p_{i}\right)$ and $W^{u}$ have transverse intersections, the Inclination Lemma implies the existence of a connected component of $\exp _{z_{i}}^{-1}\left(W^{u}\right) \cap B_{\delta_{n}}\left(z_{i}\right)$ that is the graph of a function $\psi: B_{\delta_{n}}^{u}\left(z_{i}\right) \rightarrow B_{\delta_{n}}^{s}\left(z_{i}\right)$ with $\|D \psi\| \leq \frac{1}{99}$. Let $\pi$ denote the holonomy map between $W_{\text {loc }}^{u}\left(z_{i}\right)$ and $\exp _{z_{i}}(\operatorname{graph}(\psi))$.

Sublemma 3.22. $\pi(x) \in B$ if and only if $x \in B$.

Proof. If $x \notin B$ there exist $k \geq 0$ and $\gamma^{s} \in \Gamma^{s}$ such that $f^{k} x \in \gamma^{s}$. We have $f^{k} W_{\text {loc }}^{s}(x) \subset$ $W_{\text {loc }}^{s}\left(f^{k} x\right)$ and $\gamma^{s} \subset W^{s}\left(f^{k} x\right)$. We have $W_{\text {loc }}^{s}\left(f^{k} x\right) \subset \gamma^{s}$, for otherwise $W_{\text {loc }}^{s}\left(f^{k} x\right)$ contains points that escape to infinity. Since both $x$ and $\pi(x)$ belong to $W_{\text {loc }}^{s}(x)$ then $f^{k}(\pi(x)) \in \Gamma^{s}$ so $\pi(x) \notin B$. The same reasoning yields the converse.

By Sublemma 3.22, $\pi\left(B_{i}\right) \subset B$ and Lemma 3.19 gives $\operatorname{dim}_{H}^{u}\left(\pi\left(B_{i}\right)\right) \leq \varepsilon$. (N4) yields $\operatorname{dim}_{H}^{u}\left(B_{i}\right) \leq \varepsilon$.

Proof of (P6). Take $\mu \in \mathcal{M}^{e}(f)$ with $h(\mu) \geq 2 \varepsilon$. Observe that since $\Gamma_{n} \cap W^{u}(x) \cap B \subset \bigcup_{i} B_{i}$, Lemma 3.21 yields $\operatorname{dim}_{H}^{u}\left(\Gamma_{n} \cap W^{u}(x) \cap B\right) \leq \varepsilon$ for every $n>0$, and thus $\operatorname{dim}_{H}^{u}\left(\Gamma_{\infty} \cap W^{u}(x) \cap\right.$ $B) \leq \varepsilon$. Let $\left\{\mu_{x}^{u}\right\}_{x \in \Gamma_{\infty}}$ denote the canonical system of conditional measures of $\mu$ along unstable manifolds. The dimension formula [20] gives $\operatorname{dim}\left(\mu_{x}^{u}\right)=\operatorname{dim}^{u}(\mu)=\frac{h(\mu)}{\lambda^{u}(\mu)}>\varepsilon$, and thus $\mu_{x}^{u}\left(W^{u}(x) \cap \Gamma_{\infty} \cap B\right)<1$ and $\mu_{x}^{u}\left(\left(W^{u}(x)\right)^{c} \cup \Gamma_{\infty}^{c} \cup B^{c}\right)>0$. Since $\mu_{x}^{u}\left(\left(W^{u}(x)\right)^{c}\right)=0=\mu_{x}^{u}\left(\Gamma_{\infty}^{c}\right)$ we have $\mu\left(B^{c}\right)=\int_{x \in \Gamma_{\infty}} \mu_{x}^{u}\left(B^{c}\right) d \mu(x)>0$. The $f$-invariance of $\mu$ yields $\mu(\Lambda)>0$.

Poincaré recurrence gives $\mu(X)>0$. Since $F$ is the first return map to $X$, Kac's formula [18, Theorem 2'] gives $\int_{X} \tau d \mu=1$, and so $\tau$ is $\mu$-integrable. By [46], $\mu$ is liftable.

\section{Proofs of the theorems}

In this last section we prove Theorems A, B, C, D. In Sect 4.1 we show that the induced potential $\overline{\varphi_{t}}: X \rightarrow \mathbb{R}$ has strongly summable variations and finite Gurevich pressure. Prior to Theorem A, we prove Theorem B in Sect4.2. In Sect 4.3 we define two numbers $t_{-}<0<t_{+}$ and show that $\overline{\varphi_{t}}$ is positive recurrent for any $t \in\left(t_{-}, t_{+}\right)$. From Proposition 2.4 it follows that for any $t \in\left(t_{-}, t_{+}\right)$there exists a unique measure which maximizes $F_{\varphi_{t}}$ among measures which are liftable to the inducing scheme. In Sect 4.4 we complete the proof of Theorem A by showing that this candidate measure is indeed an equilibrium measure for $\varphi_{t}$. Theorem $\mathrm{C}$ and Theorem D are proved in Sect 4.5 and Sect 4.6.

4.1. Strong summability and finite Gurevich pressure. By (P2), the unstable direction $E^{u}$ (see the definition in (2)) makes sense on each $\gamma^{u} \in \Gamma^{u}$, and coincides with its tangent directions. Hence, for each $z \in X$ we have $\sum_{i=0}^{\tau(J)-1} J^{u}\left(f^{i} z\right)=\left\|D_{z} F \mid E_{z}^{u}\right\|$. We now prove that the induced potential function $\overline{\varphi_{t}}(z):=-t \log \left\|D_{z} F \mid E_{z}^{u}\right\|$ has strongly summable variations (i.e. the potential $t \Phi=\overline{\varphi_{t}} \circ h$ has strongly summable variations). 
Lemma 4.1. There exists $C>0$ such that for every $n>0, V_{n}(\Phi) \leq C b^{-1} \sigma_{1}^{-n}$. In particular, $t \Phi$ has strongly summable variations for any $t \in \mathbb{R}$.

Proof. Take $\underline{a}, \underline{a}^{\prime} \in S^{\mathbb{Z}}$ such that $a_{i}=a_{i}^{\prime}$ provided $|i| \leq n-1$. Let $x=h(\underline{a}), x^{\prime}=h\left(\underline{a}^{\prime}\right)$. Let $y$ denote the point of intersection between $\gamma^{u}(x)$ and $\gamma^{s}\left(x^{\prime}\right)$. We have

$$
\left|\Phi(\underline{a})-\Phi\left(\underline{a^{\prime}}\right)\right|=\left|\log \frac{\left\|D_{x} F \mid E_{x}^{u}\right\|}{\left\|D_{x^{\prime}} F \mid E_{x^{\prime}}^{u}\right\|}\right| \leq\left|\log \frac{\left\|D_{x} F \mid E_{x}^{u}\right\|}{\left\|D_{y} F \mid E_{y}^{u}\right\|}\right|+\left|\log \frac{\left\|D_{y} F \mid E_{y}^{u}\right\|}{\| D_{x^{\prime}} F\left|E_{x^{\prime}}^{u}\right|}\right| .
$$

By the $F$-invariance of the $\gamma^{s}$-curves, $F^{i} y \in \gamma^{s}\left(F^{i} x^{\prime}\right)$ for $0 \leq i \leq n-1$. By the $F$-invariance of the $\gamma^{u}$-curves, $F^{i} x$ and $F^{i} y$ belong to the same $\gamma^{u}$-curves for $0 \leq i \leq n-1$. (P3) implies $|F x-F y| \leq \sigma_{1}^{-n}$. Using this and (P4)(a) we have

$$
\left|\log \frac{\left\|D_{x} F \mid E_{x}^{u}\right\|}{\left\|D_{y} F \mid E_{y}^{u}\right\|}\right| \leq C|F x-F y| \leq C \sigma_{1}^{-n} .
$$

For $z \in \Gamma^{u}$ let $e^{u}(z)$ denote the unit vector with a positive first component which spans $E_{z}^{u}$. From the bounded distortion in (P4)(b) and the proof of Lemma 3.6 in Appendix A2 we have $\left\|D_{z} f^{j} e^{u}(z)\right\| \geq(1 / 2) \kappa^{j}$ for every $j \geq 1$, where $\kappa=5^{-(1+\xi) N}$. Then the angle estimate in [44, Claim 5.3] yields

$$
\angle\left(D_{y} f^{i} e^{u}(y), D_{x^{\prime}} f^{i} e^{u}\left(x^{\prime}\right)\right) \leq(C b)^{\frac{i}{2}} \angle\left(e^{u}(y), e^{u}\left(x^{\prime}\right)\right) \leq(C b)^{\frac{i+n}{2}} .
$$

From the contraction along the $\gamma^{s}$-curves we have

$$
\left\|D_{f^{i} y} f-D_{f^{i} x^{\prime}} f\right\| \leq C\left|f^{i} y-f^{i} x^{\prime}\right| \leq(C b)^{\frac{i}{2}}\left|y-x^{\prime}\right| \leq(C b)^{\frac{i+n}{2}} .
$$

Hence

$$
\begin{aligned}
\left|\log \frac{J^{u}\left(f^{i} y\right)}{J^{u}\left(f^{i} x^{\prime}\right)}\right| & \leq C b^{-1}\left\|\frac{D_{y} f^{i+1} e^{u}(y)}{\left\|D_{y} f^{i} e^{u}(y)\right\|}-\frac{D_{x^{\prime}} f^{i+1} e^{u}\left(x^{\prime}\right)}{\left\|D_{x^{\prime}} f^{i} e^{u}\left(x^{\prime}\right)\right\|}\right\| \\
& \leq C b^{-1}\left(\left\|D_{f^{i} y} f-D_{f^{i} x^{\prime}} f\right\|+C \angle\left(D_{y} f^{i} e^{u}(y), D_{x^{\prime}} f^{i} e^{u}\left(x^{\prime}\right)\right)\right) \\
& \leq(C b)^{\frac{i+n}{2}-1} .
\end{aligned}
$$

The first inequality follows from the fact that $|\log (1+\psi)| \leq|\psi|$ for $\psi \geq 0$ and $J^{u} \geq b / 5$. The second one follows from the triangle inequality. Then

$$
\left|\log \frac{\left\|D_{y} F \mid E_{y}^{u}\right\|}{\left\|D_{x^{\prime}} F \mid E_{x^{\prime}}^{u}\right\|}\right|=\sum_{i=0}^{\tau(x)-1}\left|\log \frac{J^{u}\left(f^{i} y\right)}{J^{u}\left(f^{i} x^{\prime}\right)}\right| \leq \sum_{i=0}^{\tau\left(x_{0}\right)-1}(C b)^{\frac{i+n}{2}-1} \leq(C b)^{\frac{n}{2}-1} .
$$

(19) (20) yield the desired inequality.

We show the finiteness of the Gurevich pressure of the induced potential of a "shifted" potential. For $t, c \in \mathbb{R}$ define

$$
T_{t, c}=\sum_{J \in S} e^{c \tau(J)} \ell(J)^{t}
$$

and

$$
c_{0}(t)= \begin{cases}t \log \sigma_{2}-\varlimsup_{n \rightarrow \infty}(1 / n) \log S(n) & \text { if } t<0 \\ t \log \sigma_{1}-\varlimsup_{n \rightarrow \infty}(1 / n) \log S(n) & \text { if } t \geq 0\end{cases}
$$


By (P3)(a), for some $C>0$ we have

$$
T_{t, c} \leq\left\{\begin{array}{l}
C \sum_{n>1} S(n) e^{c n} \sigma_{2}^{-t n} \text { if } t<0 \\
C \sum_{n>1} S(n) e^{c n} \sigma_{1}^{-t n} \text { if } t \geq 0
\end{array}\right.
$$

Lemma 4.2. If $c<c_{0}(t)$, then $T_{t, c}<\infty$ and thus $P_{G}\left(\overline{\varphi_{t}+c}\right)<\infty$.

Proof. In the case $t \geq 0$, using the second alternative of (21) and (P5) we have

$$
T_{t, c} \leq C \sum_{n>1} \exp \left(n\left(c-t \log \sigma_{1}+\frac{1}{n} \log S(n)\right)\right)<\infty .
$$

The case $t<0$ can be handled similarly.

As for the Gurevich pressure, fix $J_{0} \in S$. Observe that $\overline{\varphi_{t}+c}=-t \log \left\|D f^{\tau} \mid E^{u}\right\|+c \tau$ and so,

$$
\begin{aligned}
P_{G}\left(\overline{\varphi_{t}+c}\right) & =\lim _{n \rightarrow \infty} \frac{1}{n} \log \sum_{\substack{x \in \gamma^{u}\left(\zeta_{0}\right) \cap J_{0} \\
F^{n} x \in \gamma^{s}(x)}} \exp \left(\sum_{i=0}^{n-1} \overline{\left(\varphi_{t}+c\right)}\left(F^{i} x\right)\right) \\
& \leq \lim _{n \rightarrow \infty} \frac{1}{n} \log \left(\sum_{J \in S} \sup _{x \in J} \exp \overline{\left(\varphi_{t}+c\right)}(x)\right)^{n} \leq \lim _{n \rightarrow \infty} \frac{1}{n} \log \left(C \cdot T_{t, c}\right)^{n}=\log T_{t, c}<\infty,
\end{aligned}
$$

where $C>0$ is a uniform constant.

4.2. Unstable Hausdorff dimension of $\Omega$. Before proceeding to the proof of Theorem B we need a couple of lemmas.

Lemma 4.3. $t^{u}>\frac{\log 2}{\log 5}$.

Proof. Consider the line through the two points $(0, \log 2)$ and $\left(t^{u}, 0\right)$ on the pressure curve $\left\{(t, P(t)) \in \mathbb{R}^{2}: t \in \mathbb{R}\right\}$. The point $\left(-1,\left(1 / t^{u}\right) \log 2+\log 2\right)$ lies on this line. Since the pressure curve is concave up, we have $\left(1 / t^{u}\right) \log 2+\log 2 \leq P(-1)$. Since $\|D f\|<5$ on $R$ we have $P(-1)<\log 2+\log 5$, and thus the desired inequality holds.

For $\mu \in \mathcal{M}(f)$, let

$$
\lambda^{u}(\mu)=\int \log J^{u} d \mu .
$$

A proof of the next lemma is given in Appendix A3.

Lemma 4.4. $\inf \left\{\lambda^{u}(\mu): \mu \in \mathcal{M}^{e}(f)\right\} \geq \log (2-\varepsilon)$.

Proof of Theorem B. Take an open set $\gamma$ in $W^{u}$ intersecting $\Omega$. For each $z \in \gamma \cap \Omega$ there exists a curve $\gamma(z) \subset \gamma$ and an integer $n(z)>0$ such that $f^{n(z)} \gamma(z) \in \tilde{\Gamma}^{u}$. The set $\left(f^{n(z)} \gamma(z) \cap \Omega\right) \backslash B$ is decomposed into a countable collection of sets which are sent by some positive iterates to sets of the form $\gamma^{u} \cap \Lambda, \gamma^{u} \in \tilde{\Gamma}^{u}$. By the countable stability and the invariance of the Hausdorff dimension under the action of bi-Lipschitz homeomorphisms, we have $\operatorname{dim}_{H}^{u}\left(f^{n(z)} \gamma(z) \cap \Omega\right) \backslash$ 
$B)=d^{u}(\Lambda)$. We also have $d^{u}(\Lambda)=\max \left\{d^{u}(X), d^{u}\left(\Lambda_{B}\right)\right\}$, and thus

$$
\begin{aligned}
\operatorname{dim}_{H}^{u}(\gamma(z) \cap \Omega) & =\operatorname{dim}_{H}^{u}\left(f^{n(z)} \gamma(z) \cap \Omega\right) \\
& \left.\left.=\max \left\{\operatorname{dim}_{H}^{u}\left(f^{n(z)} \gamma(z) \cap \Omega\right) \backslash B\right), \operatorname{dim}_{H}^{u}\left(f^{n(z)} \gamma(z) \cap \Omega\right) \cap B\right)\right\} \\
& \left.=\max \left\{d^{u}(\Lambda), \operatorname{dim}_{H}^{u}\left(f^{n(z)} \gamma(z) \cap \Omega\right) \cap B\right)\right\} \\
& \left.=\max \left\{d^{u}(X), d^{u}\left(\Lambda_{B}\right), \operatorname{dim}_{H}^{u}\left(f^{n(z)} \gamma(z) \cap \Omega\right) \cap B\right)\right\} .
\end{aligned}
$$

In the next two paragraphs we show $d^{u}(X)=t^{u}$. This and Lemma $4.3 \operatorname{imply} \operatorname{dim}_{H}^{u}(\gamma(z) \cap \Omega)=$ $t^{u}$. Since $\gamma$ is decomposed into a countable number of curves like $\gamma(z)$, the countable stability of Hausdorff dimension yields $\operatorname{dim}_{H}^{u}(\gamma \cap \Omega)=t^{u}$. Hence the first statement of Theorem B holds.

We are in position to show $d^{u}(X)=t^{u}$. Fix a basic element $J_{0} \in S$. Consider the covering $\mathcal{U}_{n}$ of $\gamma^{u}\left(\zeta_{0}\right) \cap J_{0}$ by $n$-cylinders. Using (P3)(a) and (P4)(a), for some $C>0$ and all $t>0$ we have

$$
\sum_{U \in \mathcal{U}_{n}} \ell(U)^{t} \leq C^{t} \sum_{\substack{x \in \gamma^{u}\left(\zeta_{0}\right) \cap J_{0} \\ F^{n} x \in \gamma^{s}(x)}} \exp \left(-t \sum_{i=0}^{n-1} \log \left\|D F \mid E^{u}\left(F^{i} x\right)\right\|\right)
$$

By definition the expression of the right-hand-side has the growth rate $P_{G}\left(\overline{\varphi_{t}}\right)$ as $n$ increases. Since the pressure is non-increasing and $t^{u}$ is the unique solution of the equation $P(t)=0$, $P(t)<0$ holds for all $t>t^{u}$. For these $t, c_{0}(t)>0$, and thus by Lemma 4.2, $\overline{\varphi_{t}}$ has finite Gurevich pressure. It has strongly summable variations by Proposition 4.1, and hence, there exists a unique $F$-invariant Gibbs measure $\nu_{\overline{\varphi_{t}}}$ for $\overline{\varphi_{t}}$. We also have $\nu_{\overline{\varphi_{t}}}(\tau)<\infty$. The Variational Principle and Abramov's and Kac's formulæ [29, Theorem 2.3] yield $P_{G}\left(\overline{\varphi_{t}}\right)<$ 0 . Hence the Hausdorff $t$-measure of $\gamma^{u}\left(\zeta_{0}\right) \cap J_{0}$ is 0 . Since $t>t^{u}$ is arbitrary, $d^{u}(X)=$ $\operatorname{dim}_{H}^{u}\left(\gamma^{u}\left(\zeta_{0}\right) \cap J_{0}\right)=\operatorname{dim}_{H}^{u}\left(\gamma^{u}\left(\zeta_{0}\right) \cap X\right) \leq t^{u}$.

To show the reverse inequality, pick an ergodic equilibrium measure for $\varphi_{t^{u}}$, which was proved to exist in [39, Theorem] and denote it by $\mu_{t^{u}}$. The dimension formula gives $h\left(\mu_{t^{u}}\right)=$ $\operatorname{dim}^{u}\left(\mu_{t^{u}}\right) \lambda^{u}\left(\mu_{t^{u}}\right)$. Using the equation $F_{\varphi_{t^{u}}}\left(\mu_{t^{u}}\right)=0, \varepsilon \ll 1$ and Lemma 4.3 we have $\operatorname{dim}^{u}\left(\mu_{t^{u}}\right)=t^{u}>4 \varepsilon$. From this and Lemma 4.4 we have $h\left(\mu_{t^{u}}\right) \geq 2 \varepsilon$. By (P6), $\mu_{t^{u}}$ is liftable. Let $\left\{\nu_{x}\right\}_{x}$ denote the canonical system of conditional measures of $\mu_{t^{u}}$ along unstable manifolds. Since $\mu_{t^{u}}$ gives full weight to the set $Y:=\bigcup_{n \geq 0} f^{n} X, \nu_{x}\left(W^{u}(x) \cap Y\right)=1$ holds for $\mu_{t^{u}}$-a.e. $x$. (P2) gives $\gamma^{u}(x) \subset W^{u}(x)$, and thus $W^{u}(x) \cap Y=\bigcup_{n \geq 0} f^{n}\left(\gamma^{u}(x) \cap X\right)$. Since $\operatorname{dim}^{u}\left(\mu_{t^{u}}\right)=\operatorname{dim}\left(\nu_{x}\right)=t^{u}$ we have $\operatorname{dim}_{H}^{u}\left(\gamma^{u}(x) \cap X\right) \geq t^{u}$, and therefore $d^{u}(X) \geq t^{u}$.

To complete the proof of Theorem B it is left to show $t^{u} \rightarrow 1$ as $b \rightarrow 0$. Define a decreasing sequence $\left\{E_{k}\right\}$ of compact sets inductively by $E_{0}=\gamma^{u}\left(\zeta_{0}\right)$ and $E_{k}=\overline{E_{k-1} \backslash f^{-k+1} \Theta_{0}}$ for $k \geq 1$. Set $E_{\infty}:=\bigcap_{k=0}^{\infty} E_{k}$. This set is similar in spirit to $\Omega_{\infty}$, but its Hausdorff dimension is easier to estimate because one removes a fixed core at each step. Observe that $E_{\infty}=$ $\overline{\gamma^{u}\left(\zeta_{0}\right) \backslash \bigcup_{i=0}^{\infty} f^{-i} \Theta_{0}} \subset \gamma^{u}\left(\zeta_{0}\right) \cap \Omega$, and $E_{\infty}$ is a Cantor set in $\gamma^{u}\left(\zeta_{0}\right)$.

Let $\mathcal{E}_{k}$ denote the collection of components of $E_{k}$. For each $A \in \mathcal{E}_{k}$ choose a point $x_{A} \in$ $A \cap E_{\infty}$ and denote by $\mu_{k}$ the atomic probability measure which is uniformly distributed on the set $\left\{x_{A}: A \in \mathcal{E}_{k}\right\}$. Pick a limit point of $\left\{\mu_{k}\right\}$ and denote it by $\mu_{\infty}$. Since $E_{\infty}$ is closed, 
$\mu_{\infty}\left(E_{\infty}\right)=1$. By construction, for every $A \in \mathcal{E}_{k}$ and $p \geq k$ we have

$$
\mu_{p}(A)=\frac{\#\left\{B \in \mathcal{E}_{p}: B \subset A\right\}}{\# \mathcal{E}_{p}}=\frac{1}{\# \mathcal{E}_{k}} .
$$

Since $\mu_{\infty}$ assigns no weight to the endpoints of $A, \mu_{p}(A) \rightarrow \mu_{\infty}(A)$ as $p \rightarrow \infty$. Hence

$$
\mu_{\infty}(A)=\lim _{p \rightarrow \infty} \mu_{p}(A)=\frac{1}{\# \mathcal{E}_{k}} .
$$

Lemma 4.5. There exist constants $C_{N}>0, C_{b}$ such that for every $k \geq 1$ and $A \in \mathcal{E}_{k}$, $\left(1 / C_{b}\right)(2+\varepsilon)^{-k} \leq \ell(A) \leq\left(1 / C_{N}\right)(2-\varepsilon)^{-k}$.

Proof. Since $b \ll 1, f$ may be viewed as a small perturbation of the Chebyshev quadratic polynomial $x \in[-1,1] \rightarrow 1-2 x^{2}$, which is topologically conjugate to the tent map with slope \pm 2 . Since the conjugacy is smooth except at the boundary points where the derivative blows up, the following holds for $f$ :

(a) there exists a constant $C_{N}>0$ such that if $i \geq 1, z \in \gamma^{u}\left(\zeta_{0}\right) \backslash \Theta_{0}$ and $f z, \ldots, f^{i-1} z \notin$ $\Theta_{0}$, then $C_{N}(2-\varepsilon)^{i} \leq\left\|D f^{i} \mid E^{u}(z)\right\| \leq C_{b}(2+\varepsilon)^{i}$.

In addition, from (a) and [44, Lemma 2.4],

(b) if $\gamma \subset R \backslash \Theta_{0}$ is a $C^{2}(b)$-curve, then $f \gamma$ is $C^{2}(b)$.

Let $A \in \mathcal{E}_{k}$. By construction, $f^{i} A$ does not intersect $\Theta_{0}$ for every $0 \leq i \leq k-1$. In particular, $f^{k-1} A$ is a $C^{2}(b)$-curve by (b). If $f^{k-1} A$ is at the left of $\Theta_{0}$, then the left endpoint of $f^{k-1} A$ is in $\tilde{\alpha}_{j}$ for some $1 \leq j \leq N-1$ and the right endpoint of it is in $\alpha_{N}^{-}$. If $f^{k-1} A$ is at the right of $\Theta_{0}$, the left endpoint of $f^{k-1} A$ is in $\alpha_{N}^{+}$and $f^{k-1} A$ intersects $\alpha_{1}^{+}$. In both cases $f^{k} A$ is a $C^{2}(b)$-curve intersecting both $f\left(\alpha_{N}^{-} \cup \alpha_{N}^{+}\right)$and $\alpha_{1}^{+}$. Therefore $\ell\left(f^{k} A\right)>L$ for some constant $L>0$ and (a) yields the desired inequalities.

Let $\operatorname{diam}\left(\Theta_{0}\right)$ denote the diameter of $\Theta_{0}$. From Lemma 3.5 (b) there exists $C>0$ independent of $\varepsilon, N, b$ such that for every $k \geq 0$,

$$
\frac{\ell\left(E_{k} \backslash E_{k+1}\right)}{\ell\left(E_{k}\right)} \leq C \operatorname{diam}\left(\Theta_{0}\right) .
$$

This yields $\ell\left(E_{k+1}\right)=\ell\left(E_{k}\right)-\ell\left(E_{k} \backslash E_{k+1}\right) \geq\left(1-C \operatorname{diam}\left(\Theta_{0}\right)\right) \ell\left(E_{k}\right)$. Using this inductively,

$$
\ell\left(E_{k}\right) \geq\left(1-C \operatorname{diam}\left(\Theta_{0}\right)\right)^{k} .
$$

Set $\rho=(2-\varepsilon)\left(1-C \operatorname{diam}\left(\Theta_{0}\right)\right)$. By (23) and Lemma 4.5,

$$
\# \mathcal{E}_{k} \geq \frac{\ell\left(E_{k}\right)}{\sup \left\{\ell(A): A \in \mathcal{E}_{k}\right\}} \geq C_{N} \rho^{k} .
$$

To finish, let $U$ be a small curve in $\gamma^{u}\left(\zeta_{0}\right)$. Choose a large integer $k>0$ such that

$$
\left(1 / C_{b}\right)(2+\varepsilon)^{-k-1}<\ell(U) \leq\left(1 / C_{b}\right)(2+\varepsilon)^{-k} .
$$

By Lemma 4.5, $U$ can intersect at most two elements of $\mathcal{E}_{k}$. Using (22), (24), (25) and $\ell(U)<1$,

$$
\begin{aligned}
\mu_{\infty}(U) & \leq\left(2 / \# \mathcal{E}_{k}\right) \leq\left(2 / C_{N}\right) \rho^{-k}=\left(2 / C_{N}\right)(2+\varepsilon)^{\frac{-k \log \rho}{\log (2+\varepsilon)}} \\
& \leq\left(2 / C_{N}\right) C_{b}^{\frac{\log \rho}{\log (2+\varepsilon)}} \ell(U)^{\frac{\log \rho}{\log (2+\varepsilon)}}
\end{aligned}
$$


The Mass Distribution Principle [14, p.60] yields $\operatorname{dim}_{H}^{u}\left(E_{\infty}\right) \geq \frac{\log \rho}{\log (2+\varepsilon)}$. Note that this number can be taken arbitrarily close to 1 at the cost of reducing $\varepsilon, 1 / N$ and $b$. Since $E_{\infty} \subset \gamma^{u}\left(\zeta_{0}\right) \cap \Omega$ and $t^{u}=\operatorname{dim}_{H}^{u}\left(\gamma^{u}\left(\zeta_{0}\right) \cap \Omega\right)$ from the first statement of Theorem $\mathrm{B}$, we obtain $t^{u} \rightarrow 1$ as $b \rightarrow 0$.

Corollary 4.6. $\lambda^{u}\left(\mu_{t^{u}}\right) \rightarrow \log 2$ as $b \rightarrow 0$.

Proof. The topological entropy of $f$ is $\log 2$. The relation $F_{\varphi_{t^{u}}}\left(\mu_{t^{u}}\right)=0$ and the variational principle give $\lambda^{u}\left(\mu_{t^{u}}\right) \leq \log 2 / t^{u}$. On the other hand, Lemma 4.4 gives $\lambda^{u}\left(\mu_{t^{u}}\right) \geq \log (2-\varepsilon)$. Since $t^{u} \rightarrow 1$ as $b \rightarrow 0$ as in Theorem B and $\varepsilon>0$ can be made arbitrarily small by choosing small $b$, we get the claim.

4.3. Positive recurrence. We now define $-1<t_{-}<0<t_{+}$by

$$
t_{+}=\frac{t^{u} \lambda^{u}\left(\mu_{t^{u}}\right)}{\lambda^{u}\left(\mu_{t^{u}}\right)-\log (2-\varepsilon)+\sqrt{\varepsilon}} \quad \text { and } \quad t_{-}=\frac{t^{u} \lambda^{u}\left(\mu_{t^{u}}\right)}{\lambda^{u}\left(\mu_{t^{u}}\right)-\log (4+\varepsilon)-\sqrt{\varepsilon}} .
$$

Corollary 4.6 implies that these definitions make sense. It also implies that one can make $t_{+}$ and $t_{-}$arbitrarily large and close to -1 respectively, by choosing sufficiently small $\varepsilon$.

Lemma 4.7. If $t \in\left(t_{-}, t_{+}\right)$, then $\overline{\varphi_{t}}$ is positive recurrent.

Proof. Let $\mathcal{M}_{L}(f)$ denote the set of liftable measures to the inducing scheme $(S, \tau)$ in Proposition 3.2 . Let

$$
P_{L}\left(\varphi_{t}\right):=\sup \left\{F_{\varphi_{t}}(\mu): \mu \in \mathcal{M}_{L}(f)\right\} .
$$

In view of Lemma 4.2 it suffices to show that one can choose $\eta_{0}>0$ so that $T_{t,-\left(P_{L}\left(\varphi_{t}\right)-\eta\right)}$ is finite for all $0 \leq \eta \leq \eta_{0}$. To show this we first estimate $P_{L}\left(\varphi_{t}\right)$ from below. In the proof of Theorem B we have shown that $\mu_{t^{u}} \in \mathcal{M}_{L}(f)$. Hence

$$
P_{L}\left(\varphi_{t}\right) \geq F_{\varphi_{t}}\left(\mu_{t^{u}}\right)=h\left(\mu_{t^{u}}\right)-t \lambda^{u}\left(\mu_{t^{u}}\right)=\left(t^{u}-t\right) \lambda^{u}\left(\mu_{t^{u}}\right) .
$$

To show the finiteness of $T_{t,-\left(P_{L}\left(\varphi_{t}\right)-\eta\right)}$ we consider the following three cases.

Case I: $0<t^{u} \leq t<t_{+}$. Using (27) and the fact that $\sigma_{1}=2-\varepsilon$ in (7) we have

$$
-P_{L}\left(\varphi_{t}\right)-t \log \sigma_{1}+\frac{1}{n} \log S(n) \leq\left(t-t^{u}\right) \lambda^{u}\left(\mu_{t^{u}}\right)-t \log (2-\varepsilon)+\frac{1}{n} \log S(n) .
$$

By the definition of $t_{+}$in (26) and the bound on $S(n)$ from (P5), the right-hand-side is strictly negative for all large $n$. Therefore for sufficiently small $\eta \geq 0$,

$$
T_{t,-\left(P_{L}\left(\varphi_{t}\right)-\eta\right)} \leq C \sum_{n>0} \exp \left(n\left(-P_{L}\left(\varphi_{t}\right)+\eta-t \log \sigma_{1}+\frac{1}{n} \log S(n)\right)\right)<\infty .
$$

Case II: $0 \leq t<t^{u}<1$. Jensen's inequality applied to the convex function $x \rightarrow x^{t}$ yields

$$
\sum_{\tau(J)=n} \ell(J)^{t} \leq S(n)^{1-t}\left(\sum_{\tau(J)=n} \ell(J)\right)^{t}
$$


Using this we have

$$
\begin{aligned}
e^{-\left(P_{L}\left(\varphi_{t}\right)-\eta\right) n} \sum_{\tau(J)=n} \ell(J)^{t} & \leq \exp \left(\left(\eta+\left(t-t^{u}\right) \lambda^{u}\left(\mu_{t^{u}}\right)+(1-t) \frac{1}{n} \log S(n)-\frac{t}{2} \log \sigma_{1}\right) n\right) \\
& \leq \exp \left(\left(\eta-t^{u} \lambda^{u}\left(\mu_{t^{u}}\right)+t\left(\lambda^{u}\left(\mu_{t^{u}}\right)-\frac{1}{2} \log \sigma_{1}\right)+(1-t) \varepsilon\right) n\right) .
\end{aligned}
$$

Since $t^{u} \rightarrow 1$ and $\lambda^{u}\left(\mu_{t^{u}}\right) \rightarrow \log 2$ as $b \rightarrow 0$, the exponent is strictly negative for sufficiently small $\eta \geq 0$. Therefore $T_{t,-\left(P_{L}\left(\varphi_{t}\right)-\eta\right)}<\infty$ holds.

Case III: $t_{-}<t \leq 0$. Using (5) and the fact that $\sigma_{2}=4+\varepsilon$ in (7) we have

$$
-P_{L}\left(\varphi_{t}\right)-t \log \sigma_{2}+\frac{1}{n} \log S(n) \leq\left(t-t^{u}\right) \lambda^{u}\left(\mu_{t^{u}}\right)-t \log (4+\varepsilon)+\frac{1}{n} \log S(n) .
$$

By the definition of $t_{-}$in (26) and (P5), the right-hand-side is strictly negative for all large $n$. Therefore for sufficiently small $\eta \geq 0$,

$$
T_{t,-\left(P_{L}\left(\varphi_{t}\right)-\eta\right)} \leq C \sum_{n>0} \exp \left(n\left(-\left(P_{L}\left(\varphi_{t}\right)-\eta\right)-t \log \sigma_{2}+\frac{1}{n} \log S(n)\right)\right)<\infty .
$$

This completes the proof of Lemma 4.7.

Corollary 4.8. For any $t \in\left(t_{-}, t_{+}\right)$there exists a unique equilibrium measure for $\varphi_{t}$ among all liftable measures.

Proof. Choose $c<c_{0}(t)$ so that $-c \gg 1$. Then $\varphi_{t}+c$ has finite Gurevich pressure, and is strongly summable by Proposition 4.1. Observe that $P_{L}\left(\varphi_{t}+c\right)=P_{L}\left(\varphi_{t}\right)+c$ and so $\overline{\varphi_{t}+c-P_{L}\left(\varphi_{t}+c\right)}=\overline{\varphi_{t}-P_{L}\left(\varphi_{t}\right)}$. Since $\varphi_{t}$ is positive recurrent by Lemma 4.7, so is $\varphi_{t}+c$. By Proposition 2.4, there exists a Gibbs measure $\nu \overline{\varphi_{t}+c}$. By the Gibbs property, for any $J \in S$ and for all $x \in J$,

$$
\nu \overline{\varphi_{t}+c}(J) \leq C \exp \left(-P_{G}\left(\overline{\varphi_{t}+c}\right)+\overline{\varphi_{t}+c}(x)\right) \leq C e^{-P_{G}\left(\overline{\varphi_{t}+c}\right)} e^{c \tau(J)} \max \left(\sigma_{1}^{-t \tau(J)}, \sigma_{2}^{-t \tau(J)}\right),
$$

and therefore

$$
\sum_{\substack{J \in S \\ \tau(J)=n}} \tau(J) \nu \overline{\varphi+c}(J) \leq C n S(n) e^{-P_{G}\left(\overline{\varphi_{t}+c}\right)} e^{c n} \max \left(\sigma_{1}^{-t n}, \sigma_{2}^{-t n}\right) .
$$

The right-hand-side has a negative growth rate as $n$ increases. Hence $\nu_{\overline{\varphi_{t}+c}}(\tau)<\infty$ holds. By Proposition 2.4, there exists a unique equilibrium measure for $\varphi_{t}+c$ among all liftable measures. Since $\varphi_{t}+c$ is cohomologous to $\varphi_{t}$, they yield the same equilibrium measures.

4.4. Uniqueness of equilibrium measures for $\varphi_{t}$. We finish the proof of Theorem A. We start with preliminary estimates of $t_{ \pm}$. Define

$$
\lambda_{M}^{u}:=\sup \left\{\lambda^{u}(\mu): \mu \in \mathcal{M}^{e}(f)\right\} \text { and } \lambda_{m}^{u}:=\inf \left\{\lambda^{u}(\mu): \mu \in \mathcal{M}^{e}(f)\right\} .
$$

Lemma 4.9. We have

$$
t_{+}<\frac{t^{u} \lambda^{u}\left(\mu_{t^{u}}\right)-2 \varepsilon}{\lambda^{u}\left(\mu_{t^{u}}\right)-\lambda_{m}^{u}} \text { and } t_{-}>\frac{t^{u} \lambda^{u}\left(\mu_{t^{u}}\right)-2 \varepsilon}{\lambda^{u}\left(\mu_{t^{u}}\right)-\lambda_{M}^{u}} .
$$


Proof. A direct computation gives

$$
\frac{t^{u} \lambda^{u}\left(\mu_{t^{u}}\right)-2 \varepsilon}{\lambda^{u}\left(\mu_{t^{u}}\right)-\lambda_{m}^{u}}-t_{+}=\frac{t^{u} \lambda^{u}\left(\mu_{t^{u}}\right)\left(\lambda_{m}^{u}-\log (2-\varepsilon)+\sqrt{\varepsilon}\right)-2 \varepsilon\left(\lambda^{u}\left(\mu_{t^{u}}\right)-\log (2-\varepsilon)+\sqrt{\varepsilon}\right)}{\left(\lambda^{u}\left(\mu_{t^{u}}\right)-\lambda_{m}^{u}\right)\left(\lambda^{u}\left(\mu_{t^{u}}\right)-\log (2-\varepsilon)+\sqrt{\varepsilon}\right)} .
$$

The denominator of the fraction of the right-hand-side is positive. Since $t^{u} \rightarrow 1$ and $\lambda^{u}\left(\mu_{t^{u}}\right) \rightarrow$ $\log 2$ as $b \rightarrow 0$, the first term of the numerator is $\geq(1 / 2) \sqrt{\varepsilon}$. Hence the numerator is positive, and the first inequality holds. A proof of the second one is analogous.

Proof of Theorem $A$. Given a bounded interval $I \subset(-1, \infty)$, choose $\varepsilon$ and $b$ so that $I \subset$ $\left(t_{-}, t_{+}\right)$. Let $t \in I$. In view of Corollary 4.8 we need to consider measures which are not liftable to the inducing scheme $(S, \tau)$. Since

$$
\sup \left\{F_{\varphi_{t}}(\mu): \mu \in \mathcal{M}(f) \backslash \mathcal{M}_{L}(f)\right\}=\sup \left\{F_{\varphi_{t}}(\mu): \mu \in \mathcal{M}^{e}(f) \backslash \mathcal{M}_{L}(f)\right\},
$$

we may restrict ourselves to ergodic measures. It suffices to show

$$
\sup \left\{F_{\varphi_{t}}(\mu): \mu \in \mathcal{M}^{e}(f) \backslash \mathcal{M}_{L}(f)\right\}<P_{L}\left(\varphi_{t}\right) .
$$

We argue by contradiction assuming (29) is false. Then, for any $\delta>0$ there exists $\mu \in$ $\mathcal{M}^{e}(f) \backslash \mathcal{M}_{L}(f)$ such that $h(\mu)-t \lambda^{u}(\mu) \geq P_{L}\left(\varphi_{t}\right)-\delta$. Then

$$
h(\mu) \geq t\left(\lambda^{u}(\mu)-\lambda^{u}\left(\mu_{t^{u}}\right)\right)+t^{u} \lambda^{u}\left(\mu_{t^{u}}\right)-\delta .
$$

For the rest of the proof we deal with two cases separately.

Case I: $0 \leq t<t_{+}$. We have $h(\mu) \geq t\left(\lambda_{m}^{u}-\lambda^{u}\left(\mu_{t^{u}}\right)\right)+t^{u} \lambda^{u}\left(\mu_{t^{u}}\right)-\delta$. Since $\delta>0$ is arbitrary we get

$$
h(\mu) \geq t\left(\lambda_{m}^{u}-\lambda^{u}\left(\mu_{t^{u}}\right)\right)+t^{u} \lambda^{u}\left(\mu_{t^{u}}\right) .
$$

(30) and the first inequality in Lemma 4.9 yield $h(\mu)>2 \varepsilon$. (P6) gives $\mu \in \mathcal{M}_{L}(f)$, which is a contradiction.

Case II: $t_{-}<t<0$. Follows similarly from the second inequality in Lemma 4.9.

4.5. Measure of maximal unstable dimension. We now prove the existence and uniqueness of a measure of maximal unstable dimension.

Proof of Theorem $C$. Let $\mu \in \mathcal{M}^{e}(f)$. If $\mu \in \mathcal{M}_{L}(f)$, then $\mu\left(\bigcup_{n \geq 0} f^{n} X\right)=1$. Arguing similarly to the last paragraph in the proof of Theorem B we obtain $\operatorname{dim}^{u}(\mu) \leq d(X)=t^{u}$. If $\mu \notin \mathcal{M}_{L}(f)$, then $(\mathrm{P} 6)$ gives $h(\mu)<2 \varepsilon$, and since $t^{u} \rightarrow 1$ as $b \rightarrow 0$, we have $\operatorname{dim}^{u}(\mu)<t^{u}$ for $b, \varepsilon$ small enough. Since $\operatorname{dim}^{u}\left(\mu_{t^{u}}\right)=t^{u}, \mu_{t^{u}}$ is a measure of maximal unstable dimension.

As for the uniqueness, let $\mu$ be a measure of maximal unstable dimension. Then $\operatorname{dim}^{u}(\mu)=$ $t^{u}$, and so $h(\mu)-t^{u} \lambda^{u}(\mu)=0$, namely $\mu$ is an equilibrium measure for $\varphi_{t^{u}}$. The uniqueness in Theorem A yields $\mu=\mu_{t^{u}}$.

4.6. Statistical properties of equilibrium measures. We now prove statistical properties of $\mu_{t}$.

Proof of Theorem D. Once the existence of the equilibrium measure for $\varphi_{t}$ is established, the statistical properties in Theorem D can be deduced from the abstract results of Young [45, Theorems 2 and 3] with the exponential tail estimate in (28). 


\section{APPENDIX: COMPUTATIONAL PROOFS}

We refer the reader to [39, Sect.2] for relevant definitions and results used in this appendix.

A1. Proof of Lemma 3.5. Let $\zeta$ denote the critical point [39, Sect.2.2] on $\gamma$, and $p(z)$ the corresponding bound period for $z \in \gamma_{n}$ [39, Sect.2.3]. By [39, Proposition 2.5], $\left\|D_{z} f^{p(z)} \mid E_{z}^{u}\right\| \geq$ $(4-2 \varepsilon)^{\frac{p(z)}{2}}$ and slope $\left(D_{z} f^{p(z)} \mid E_{z}^{u}\right) \leq \sqrt{b}$. Since $p(z)<n$, the derivative estimate in [39, Lemma 2.3] gives $\left\|D_{f^{p(z)} z} f^{n-p(z)} \mid E_{f^{p(z)} z}^{u}\right\| \geq \sigma_{1}^{n-p(z)}$. Hence $\left\|D_{z} f^{n} \mid E_{z}^{u}\right\| \geq(4-2 \varepsilon)^{\frac{p(z)}{2}} \sigma_{1}^{n-p(z)} \geq \sigma_{1}^{n}$ and (a) holds.

For $z \in \gamma_{n}$, Let $e^{u}(z)$ denote the unit vector which spans $E_{z}^{u}$ and has a positive first component. Consider the stable foliation $\mathcal{F}^{s}$ [39, Sect.2.2], and let $\mathcal{F}^{s}(f z)$ denote the leaf through $f z$. Let $e^{s}(f z)$ denote the unit vector which spans $T_{f z} \mathcal{F}^{s}(f z)$ and has a positive second component. Split $D_{z} f e^{u}(z)=A(z)\left(\begin{array}{l}1 \\ 0\end{array}\right)+B(z) e^{s}(f z)$. [41, Lemma 2.2] gives

$$
|A(z)| \approx|\zeta-z| \text { and }|B(z)| \leq C \sqrt{b}
$$

Let $p=\max \left\{p(z): z \in \gamma_{n}\right\}$. Split $\left\|D_{x} f^{p} e^{u}(x)-D_{y} f^{p} e^{u}(y)\right\| \leq I_{1}+I_{2}+I_{3}+I_{4}$, where

$$
\begin{aligned}
I_{1} & =|A(x)-A(y)| \cdot\left\|D_{f x} f^{p-1}\left(\begin{array}{l}
1 \\
0
\end{array}\right)\right\|, \\
I_{2} & =|B(x)-B(y)| \cdot\left\|D_{f x} f^{p-1} e^{s}(f x)\right\|, \\
I_{3} & =|B(y)| \cdot\left\|D_{f x} f^{p-1} e^{s}(f x)-D_{f y} f^{p-1} e^{s}(f y)\right\|, \\
I_{4} & =|A(y)| \cdot\left\|D_{f x} f^{p-1}\left(\begin{array}{l}
1 \\
0
\end{array}\right)-D_{f y} f^{p-1}\left(\begin{array}{l}
1 \\
0
\end{array}\right)\right\| .
\end{aligned}
$$

Estimates of $I_{1}, I_{2}$. Let $e^{s}(z)=\left(\begin{array}{c}e_{1}(z) \\ e_{2}(z)\end{array}\right)$, and

$$
S(z)=\left(\begin{array}{cc}
1 & e_{1}(z) \\
0 & e_{2}(z)
\end{array}\right)^{-1}=\left(\begin{array}{cc}
1+\epsilon_{1} & \epsilon_{2} \\
\epsilon_{3} & 1+\epsilon_{4}
\end{array}\right) \text { and } D_{z} f=\left(\begin{array}{cc}
-2 a^{*} z_{1}+\alpha_{1} & \alpha_{2} \\
\alpha_{3} & \alpha_{4}
\end{array}\right)
$$

where $z_{1}$ denotes the first coordinate of $z$. Let $R(z)$ denote the rotation matrix by $\theta(z):=$ $\angle\left(e^{u}(z),\left(\begin{array}{l}1 \\ 0\end{array}\right)\right)$. Then $A(z), B(z)$ are equal to the $(1,1),(2,1)$ entries of the matrix $S(z) \cdot D_{z} f$. $R(z)^{-1}$ correspondingly. A direct computation shows that $A(z), B(z)$ are linear combinations of $\alpha_{i}, \epsilon_{i}(1 \leq i \leq 4), \cos \theta, \sin \theta$, all of which are Lipschitz continuous on $\gamma_{n}$, from (1), property (F3) of $\mathcal{F}^{s}$ in [39, Sect.2.2] and the $C^{2}(b)$-property of $\gamma_{n}$. Hence $A(z), B(z)$ are Lipschitz continuous on $\gamma_{n}$ as well, which implies

$$
I_{1} \leq C|x-y| \cdot\left\|w_{p}(\zeta)\right\| \quad \text { and } \quad I_{2} \leq(C b)^{p-1}|x-y| \cdot
$$

Estimate of $I_{3}$. We start with an elementary geometric reasoning. Let $v_{1}, v_{2}$ be nonzero vectors in $\mathbb{R}^{2}$ such that $\left\|v_{1}\right\| \leq\left\|v_{2}\right\|, \theta \ll 1$ (See figure 3 ). We have

$$
\begin{aligned}
\left\|v_{2}-v_{1}\right\| & <\left|\left\|v_{2}\right\|-\left\|v_{1}\right\| \cos \theta\right|+\left\|v_{1}\right\| \sin \theta \\
& =\cos \theta\left|\left\|v_{2}\right\|-\left\|v_{1}\right\|\right|+(1-\cos \theta)\left\|v_{2}\right\|+\left\|v_{1}\right\| \sin \theta \\
& \leq\left|\left\|v_{2}\right\|-\left\|v_{1}\right\|\right|+2 \theta\left\|v_{2}\right\| .
\end{aligned}
$$

Without loss of generality we may assume $\left\|D_{y} f^{p-1} e^{s}(y)\right\| \geq\left\|D_{x} f^{p-1} e^{s}(x)\right\|$. The angle between the two vectors involved in $I_{3}$ is small. The fact that $|B(y)| \leq C$ and the above reasoning show

$$
I_{3} \leq C\left\|D_{y} f^{p-1} e^{s}(y)\right\|\left(\left|\frac{\left\|D_{x} f^{p-1} e^{s}(x)\right\|}{\left\|D_{y} f^{p-1} e^{s}(y)\right\|}-1\right|+3\left\|e^{s}\left(f^{p} x\right)-e^{s}\left(f^{p} y\right)\right\|\right) .
$$


To estimate the first term in the parenthesis of (33) we argue as follows. Let $J^{s}(z)=$ $\left\|D_{z} f e^{s}(z)\right\|$. The invariance of the stable foliation $\mathcal{F}^{s}$ gives

$$
\log \frac{\left\|D_{x} f^{p-1} e^{s}(x)\right\|}{\left\|D_{y} f^{p-1} e^{s}(y)\right\|} \leq \sum_{i=1}^{p-1} \log \frac{J^{s}\left(f^{i} x\right)}{J^{s}\left(f^{i} y\right)}
$$

Let $e^{s \perp}(z)$ denote any unit vector orthogonal to $e^{s}(z), \theta(z)=\angle\left(D_{z} f e^{s}(z), D_{z} f e^{s \perp}(z)\right)$, and let $J^{s \perp}(z)=\left\|D_{z} f e^{s \perp}(z)\right\|$. Then $e^{s \perp}$ and $\theta$ are Lipschitz continuous, $\theta \approx \pi / 2$ and $J^{s \perp}>2$. Hence $\log J^{s \perp}$ and $\sin \theta$ are Lipschitz continuous, with Lipschitz constants independent of $b$. Since $J^{s}\left(f^{i} x\right) J^{s \perp}\left(f^{i} x\right) \sin \theta\left(f^{i} x\right)=\left|\operatorname{det} D_{f^{i} x} f\right|=b$, for $1 \leq i<p$ we have

$$
\log \frac{J^{s}\left(f^{i} x\right)}{J^{s}\left(f^{i} y\right)}=\log \frac{J^{s \perp}\left(f^{i} y\right)}{J^{s^{\perp}}\left(f^{i} x\right)}+\log \frac{\sin \theta\left(f^{i} y\right)}{\sin \theta\left(f^{i} x\right)} \leq C\left|f^{i} x-f^{i} y\right| .
$$

Sublemma 4.10. $\sum_{i=1}^{p-1}\left|f^{i} x-f^{i} y\right| \leq C\left|f^{p} x-f^{p} y\right|+\frac{C}{\sqrt{b}} \frac{|x-y|}{d\left(\zeta, \gamma_{n}\right)}$.

Proof. We introduce a new coordinate $(\xi, \eta)$ as follows: for a point $z=\left(z_{1}, z_{2}\right) \in \mathbb{R}^{2}$ let

$$
\left(z_{1}, z_{2}\right)=(\xi(z), 0)+\left(\eta(z) e_{1}^{s}(f \zeta), \eta(z) e_{2}^{s}(f \zeta)\right) .
$$

Note that there exist $C_{1}>C_{2}>0$ such that

$$
C_{2} \leq \frac{\left|z-z^{\prime}\right|}{\left|\xi(z)-\xi\left(z^{\prime}\right)\right|+\left|\eta(z)-\eta\left(z^{\prime}\right)\right|} \leq C_{1} .
$$

Parametrize $\gamma_{n}$ by arc length $s$ and let $\hat{\gamma}(s)=f\left(\gamma_{n}(s)\right)$. Let $x=\gamma_{n}\left(s_{1}\right), y=\gamma_{n}\left(s_{2}\right)$, and assume $s_{1}<s_{2}$ without loss of generality. For $s \in\left[s_{1}, s_{2}\right]$ define two numbers $\tilde{A}(s), \tilde{B}(s)$ by $\left.\frac{d \hat{\gamma}}{d t}\right|_{t=s}=\tilde{A}(s)\left(\begin{array}{l}1 \\ 0\end{array}\right)+\tilde{B}(s) e^{s}(f \zeta)$. (31) and $\left|e^{s}(f \zeta)-e^{s}(f z)\right| \leq C|\zeta-z|$ together imply $|\tilde{A}(s)| \approx$ $\left|\zeta-\gamma_{n}(s)\right|$ and $|\tilde{B}(s)| \leq C \sqrt{b}$. Since $\left|\zeta-\gamma_{n}(s)\right| \geq d\left(\zeta, \gamma_{n}\right)$ we have $\left|\xi\left(\gamma\left(s_{1}\right)\right)-\xi\left(\gamma\left(s_{2}\right)\right)\right|=$ $\left|\int_{s_{1}}^{s_{2}} \tilde{A}(s) d s\right| \geq\left|s_{1}-s_{2}\right| C d\left(\zeta, \gamma_{n}\right)$, and $\left|\eta\left(\gamma\left(s_{1}\right)\right)-\eta\left(\gamma\left(s_{2}\right)\right)\right|=\left|\int_{s_{1}}^{s_{2}} \tilde{B}(\gamma(s)) d s\right| \leq C \sqrt{b}\left|s_{1}-s_{2}\right|$. Namely,

$$
|\xi(f x)-\xi(f y)| \geq C d\left(\zeta, \gamma_{n}\right)|x-y| \text { and }|\eta(f x)-\eta(f y)| \leq C \sqrt{b}|x-y| .
$$

For $1 \leq i \leq p-1$ we have $\left|\xi\left(f^{i} x\right)-\xi\left(f^{i} y\right)\right| \leq \sigma_{1}^{-(i-p)}\left|\xi\left(f^{p} x\right)-\xi\left(f^{p} y\right)\right|$, and

$$
\left|\eta\left(f^{i} x\right)-\eta\left(f^{i} y\right)\right| \leq(C b)^{\frac{i-1}{2}}|\eta(f x)-\eta(f y)| \leq \frac{(C b)^{\frac{i-1}{2}}}{\sqrt{b}} \frac{|\xi(f x)-\xi(f y)|}{d\left(\zeta, \gamma_{n}\right)} \leq \frac{(C b)^{\frac{i-1}{2}}}{\sqrt{b}} \frac{|x-y|}{d\left(\zeta, \gamma_{n}\right)},
$$

where the second inequality follows from (35). Summing these two inequalities over all $1 \leq$ $i \leq p-1$ yields the desired one.

Sublemma 4.10 implies that the right-hand-side of (34) is bounded by a constant $C>0$ independent of $b$. Since there exists $\rho=\rho(C)>0$ such that $e^{\psi} \leq 1+\rho \psi$ for $0 \leq \psi \leq C$, we have

$$
\frac{\left\|D_{x} f^{p-1} e^{s}(x)\right\|}{\left\|D_{y} f^{p-1} e^{s}(y)\right\|}-1 \leq \rho \sum_{i=1}^{p-1} \log \frac{J^{s}\left(f^{i} x\right)}{J^{s}\left(f^{i} y\right)} \leq \rho C \sum_{i=1}^{p-1}\left|f^{i} x-f^{i} y\right| .
$$

\footnotetext{
${ }^{1}$ Here we use the fact that the Jacobian of the Hénon map is constant equal to $b$. Essentially the same argument remains to hold for Hénon-like maps for which there exists $C>0$ independent of $b$ such that $\|D \log |\operatorname{det} D f|\| \leq C$ (c.f. [26]). Therefore our main theorems hold for Hénon-like maps satisfying this assumption.
} 


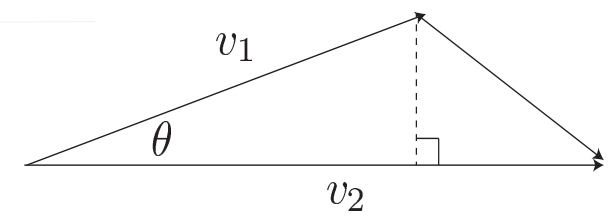

Figure 8. $\left\|v_{1}\right\| \leq\left\|v_{2}\right\|, \theta \ll 1$

We are in position to finish the estimate of $I_{3}$. We have $\left\|D_{y} f^{p-1} e^{s}(y)\right\| \leq C b$, and the second term in the parenthesis in (33) is $\leq C\left|f^{p} x-f^{p} y\right|$. Then, combining Sublemma 4.10, (36) and plugging the result into (33) yields

$$
I_{3} \leq C b\left|f^{p} x-f^{p} y\right|+C \frac{|x-y|}{d\left(\zeta, \gamma_{n}\right)} .
$$

Estimate of $I_{4}$. In the same way as in the proof of (33) we have

$$
I_{4} \leq|A(y)| \cdot\left\|w_{p}(\zeta)\right\|\left(\left|\frac{\left\|D_{f x} f^{p-1}\left(\begin{array}{l}
1 \\
0
\end{array}\right)\right\|}{\left\|D_{f y} f^{p-1}\left(\begin{array}{l}
1 \\
0
\end{array}\right)\right\|}-1\right|+2 \angle\left(D_{f x} f^{p-1}\left(\begin{array}{l}
1 \\
0
\end{array}\right), D_{f y} f^{p-1}\left(\begin{array}{l}
1 \\
0
\end{array}\right)\right)\right) .
$$

From the distortion estimate in the proof of [39, Lemma 2.7] and Sublemma 4.10, the first term in the parenthesis is $\leq C\left|f^{p} x-f^{p} y\right|$. To estimate the second term in the parenthesis, take a point $r$ so that the leaf $\mathcal{F}^{s}(f y)$ intersects the horizontal through $f x$ at $f r$. By the angle estimate in [44, Claim 5.3],

$$
\angle\left(D_{f y} f^{p-1}\left(\begin{array}{l}
1 \\
0
\end{array}\right), D_{f r} f^{p-1}\left(\begin{array}{l}
1 \\
0
\end{array}\right)\right) \leq(C b)^{p-1}|f y-f r| \leq(C b)^{p-1}|x-y| \leq(C b)^{p-1}\left|f^{p} x-f^{p} y\right| .
$$

By the $C^{2}(b)$-property and the definition of $r$,

$$
\angle\left(D_{f x} f^{p-1}\left(\begin{array}{l}
1 \\
0
\end{array}\right), D_{f r} f^{p-1}\left(\begin{array}{l}
1 \\
0
\end{array}\right)\right) \leq \sqrt{b}\left|f^{p} x-f^{p} r\right| \leq C \sqrt{b}\left|f^{p} x-f^{p} y\right| .
$$

Hence we obtain

$$
\angle\left(D_{f x} f^{p-1}\left(\begin{array}{l}
1 \\
0
\end{array}\right), D_{f y} f^{p-1}\left(\begin{array}{l}
1 \\
0
\end{array}\right)\right) \leq C \sqrt{b}\left|f^{p} x-f^{p} y\right| .
$$

Additionally (31) yields

$$
|A(y)| \leq C|\zeta-y| \leq C\left(d\left(\zeta, \gamma_{n}\right)+\ell\left(\gamma_{n}\right)\right)
$$

where $d(\cdot, \cdot)$ denotes the minimal distance apart. Finally, from Sublemma 4.11 below we get

$$
I_{4} \leq C d\left(\zeta, \gamma_{n}\right)\left\|w_{p}(\zeta)\right\| \cdot\left|f^{p} x-f^{p} y\right|
$$

Sublemma 4.11. $\ell\left(\gamma_{n}\right) \leq C d\left(\zeta, \gamma_{n}\right)$.

Proof. Let $M$ be a large integer such that $M \ll N$. Consider the leaf of the stable foliation $\mathcal{F}^{s}$ through $f \zeta$ which is of the form $\mathcal{F}^{s}(f \zeta)=\{(x(y), y):|y| \leq \sqrt{b}\}$. For $k>M$ define

$$
U_{k}:=\left\{(x, y): D_{k} \leq|x-x(y)|<D_{k-M},|y| \leq \sqrt{b}\right\}
$$

where $D_{k}:=C\left[\sum_{i=1}^{k} \frac{\left\|w_{i}(\zeta)\right\|^{2}}{\left\|w_{i+1}(\zeta)\right\|}\right]^{-1}$ for some constant $C>0$. Let $k_{0}:=\max \left\{k>M: U_{k} \cap f \gamma_{n} \neq\right.$ $\emptyset\}-1$. By [39, Lemma 2.5(a)], there exist constants $0<C_{1}<C_{2}<1 / 2$ such that

$$
C_{1} D_{k_{0}-M} \leq D_{k_{0}} \leq C_{2} D_{k_{0}-M}
$$


We prove

$$
f \gamma_{n} \subset U_{k_{0}} \cup U_{k_{0}+1}
$$

(39) (40) imply $\ell\left(\gamma_{n}\right) \leq C \sqrt{D_{k_{0}-M}} \leq C \sqrt{D_{k_{0}}} \leq C d\left(\gamma_{n}, \zeta\right)$, and thus Sublemma 4.11 holds.

It is left to prove (40). If the inclusion were false, then one could choose a curve $\delta \subset f \gamma_{n} \cap U_{k_{0}}$ with endpoints in the two vertical boundaries of $U_{k_{0}}$. Let $x$ denote the endpoint of $f^{2} \gamma_{n}$ in $\tilde{\alpha}_{n-1}$. The bounded distortion and the second inequality in [39, Lemma 2.5(b)] give

$$
d\left(\alpha_{0}^{-}, f^{k_{0}-M} x\right) \leq 2 D_{k_{0}}\left\|w_{k_{0}-M+1}(\zeta)\right\| \leq 2 \cdot 3^{-M} D_{k_{0}}\left\|w_{k_{0}}(\zeta)\right\| \leq 3^{-M},
$$

and

$$
\ell\left(f^{k_{0}-M} \delta\right) \geq C\left(D_{k_{0}-M}-D_{k_{0}}\right)\left\|w_{k_{0}-M}(\zeta)\right\| \geq C\left(1-C_{2}\right) D_{k_{0}-M}\left\|w_{k_{0}-M}(\zeta)\right\| \geq C .
$$

From these two estimates and choosing large $M$ if necessary we have that the interior of $f^{k-10} \delta$ intersects some $\tilde{\alpha}_{i}$. This yields a contradiction.

Overall estimates. Gluing (32) (37) (38) together,

$$
\left\|D_{x} f^{p} e^{u}(x)-D_{y} f^{p} e^{u}(y)\right\| \leq C\left\|w_{p}(\zeta)\right\| \cdot|x-y|+\frac{C|x-y|}{d\left(\zeta, \gamma_{n}\right)}+C d\left(\zeta, \gamma_{n}\right)\left\|w_{p}(\zeta)\right\| \cdot\left|f^{p} x-f^{p} y\right| .
$$

From the proof of [39, Proposition 2.6] there exists $C>0$ such that $\left\|D_{z} f^{p} e^{u}(z)\right\| \geq C d\left(\zeta, \gamma_{n}\right)$. $\left\|w_{p}(\zeta)\right\| \geq 1$ for $z=x, y$. Hence

$$
\log \frac{\left\|D_{x} f^{p} \mid E_{x}^{u}\right\|}{\left\|D_{y} f^{p} \mid E_{y}^{u}\right\|} \leq \frac{C|x-y|}{d\left(\zeta, \gamma_{n}\right)}+C\left|f^{p} x-f^{p} y\right| .
$$

[39, Proposition 2.5(c)(d)] and [44, Lemma 2.4] together imply that $f^{p} \gamma_{n}$ is a $C^{2}(b)$-curve. By the uniform hyperbolicity outside of $\Theta, f^{p+1} \gamma_{n}, \ldots, f^{n-1} \gamma_{n}$ are $C^{2}(b)$ as well and we have

$$
\left|f^{p} x-f^{p} y\right| \leq\left|f^{n} x-f^{n} y\right| \text { and } \log \frac{\left\|D_{f^{p} x} f^{n-p} \mid E_{f^{p} x}^{u}\right\|}{\left\|D_{f^{p} y} f^{n-p} \mid E_{f^{p} y}^{u}\right\|} \leq C\left|f^{n} x-f^{n} y\right| .
$$

(41) (42) and Sublemma 4.12 below yield

$$
\log \frac{\left\|D_{x} f^{n} \mid E_{x}^{u}\right\|}{\left\|D_{y} f^{n} \mid E_{y}^{u}\right\|}=\log \frac{\left\|D_{x} f^{p} \mid E_{x}^{u}\right\|}{\left\|D_{y} f^{p} \mid E_{y}^{u}\right\|}+\log \frac{\left\|D_{f^{p} x} f^{n-p} \mid E_{f^{p} x}^{u}\right\|}{\left\|D_{f^{p} y} f^{n-p} \mid E_{f^{p} y}^{u}\right\|} \leq C\left|f^{n} x-f^{n} y\right|,
$$

which proves (b).

Sublemma 4.12. $\frac{|x-y|}{d\left(\zeta, \gamma_{n}\right)} \leq C\left|f^{n} x-f^{n} y\right|$.

Proof. By the bounded distortion outside of $\Theta$, there exists $\theta \in f \gamma_{n}$ such that

$$
|\xi(f x)-\xi(f y)| \cdot\left\|D_{\theta} f^{n-1}\left(\begin{array}{l}
1 \\
0
\end{array}\right)\right\| \leq C\left|f^{n} x-f^{n} y\right| .
$$

The bounded distortion outside of $\Theta$ and the quadratic behavior near $\zeta$ as in (31) imply

$$
\ell\left(\gamma_{n}\right) d\left(\zeta, \gamma_{n}\right)\left\|D_{\theta} f^{n-1}\left(\begin{array}{l}
1 \\
0
\end{array}\right)\right\| \geq C \ell\left(f^{n} \gamma_{n}\right) .
$$

Hence there exists $C>0$ such that

$$
d\left(\zeta, \gamma_{n}\right)^{2}\left\|D_{\theta} f^{n-1}\left(\begin{array}{l}
1 \\
0
\end{array}\right)\right\| \geq C \ell\left(\gamma_{n}\right) d\left(\zeta, \gamma_{n}\right)\left\|D_{\theta} f^{n-1}\left(\begin{array}{l}
1 \\
0
\end{array}\right)\right\| \geq C \ell\left(f^{n} \gamma_{n}\right)>C .
$$


The first inequality follows from Sublemma 4.11, and the last inequality is because $f^{n} \gamma_{n}$ is a $C^{2}(b)$-curve with endpoints in $\alpha_{1}^{ \pm}$. Using the first inequality and (35) and then (43) (44) yield

$$
\frac{|x-y|}{d\left(\zeta, \gamma_{n}\right)} \leq \frac{C|\xi(f x)-\xi(f y)|}{d\left(\zeta, \gamma_{n}\right)^{2}} \leq \frac{C\left|f^{n} x-f^{n} y\right|}{d\left(\zeta, \gamma_{n}\right)^{2}\left\|D_{\theta} f^{n-1}\left(\begin{array}{l}
1 \\
0
\end{array}\right)\right\|} \leq C\left|f^{n} x-f^{n} y\right| .
$$

\section{A2. Proof of Lemma 3.6. Set $\kappa=5^{-(1+\xi) N}$.}

Sublemma 4.13. For any $z \in \Omega_{\infty}$ and every $n \geq 1,\left\|D_{z} f^{n} \mid E_{z}^{u}\right\| \geq \kappa^{n}$.

Proof. With the terminology in [39, Sect.2.5] we introduce the bound/free structure on the orbit of $z$, using $\Theta_{0}$ as a critical neighborhood. If $f^{n} z$ is free, then the orbit $z, \ldots, f^{n} z$ is decomposed into alternative bound and free segments. Applying the expansion estimates in [39, Lemma 2.3, Proposition 2.5] alternatively we have $\left\|D_{z} f^{n} \mid E_{z}^{u}\right\| \geq \kappa^{n}$. If $f^{n} z$ is bound, then there exists an integer $0<m<n$ such that $f^{m} z \in \Theta_{0}$ and $m<n<m+p$, where $p$ is the bound period of $f^{m} z$. Since $f^{m+p} z$ is free and $\|D f\|<5$ we have $\left\|D_{z} f^{n} \mid E_{z}^{u}\right\| \geq$ $5^{-(m+p-n)}\left\|D_{z} f^{m+p} \mid E_{z}^{u}\right\|>5^{-p}$, and since $z \in \Omega_{\infty}$ we have $p \leq \xi m+N \leq \xi n+N$ and so $\left\|D_{z} f^{n} \mid E_{z}^{u}\right\| \geq 5^{-\xi n-N^{2}} \geq \kappa^{n}$.

From Sublemma 4.13 and the results in [26, Sect.6, Sect.7C], there exists a long stable leaf through $z$. The uniqueness follows from the next sublemma with $n=0$.

Sublemma 4.14. Let $z_{1}, z_{2} \in \Omega_{\infty}$ and let $\gamma^{s}\left(z_{i}\right)$ denote any long stable leaf through $z_{i}(i=$ $1,2)$. If $f^{n}\left(\gamma^{s}\left(z_{1}\right)\right) \cap \gamma^{s}\left(z_{2}\right) \neq \emptyset$ for some $n \geq 0$, then $f^{n} \gamma^{s}\left(z_{1}\right) \subset \gamma^{s}\left(z_{2}\right)$.

Proof. Choose a large integer $M \gg n$ such that $f^{M} z_{2}$ is free. Take $x_{1} \in f^{n} \gamma^{s}\left(z_{1}\right), x_{2} \in \gamma^{s}\left(z_{2}\right)$ which are connected by a horizontal segment of length $b^{\frac{M}{3}}$. By construction, $\left\|D_{x_{2}} f^{M} \mid E_{x_{2}}^{u}\right\| \geq$ $\sigma_{1}^{M}$. By the bounded distortion, the $f^{M}$-iterate of the segment is $C^{2}(b)$ and $\left|f^{M} x_{1}-f^{M} x_{2}\right| \geq$ $C \sigma_{1}^{M}\left|x_{1}-x_{2}\right| \geq C \sigma_{1}^{M} b^{\frac{M}{3}}$. If $q \in f^{n} \gamma^{s}\left(z_{1}\right) \cap \gamma^{s}\left(z_{2}\right)$, then $\left|f^{M} x_{1}-f^{M} x_{2}\right| \leq\left|f^{M} x_{1}-f^{M} q\right|+$ $\left|f^{M} q-f^{M} x_{2}\right| \leq 2(C b)^{\frac{M}{2}}$. These two estimates are incompatible.

Lemma 3.6(a) is a consequence of Sublemma 4.14. Since the stable sides of $\Theta$ are long stable leaves, $\gamma^{s}(z) \subset \Theta$ follows from the disjointness in Lemma 3.6(a). The rest of the items in the lemma follow from the results in [26, Sect.6, Sect.7C] and [4, Proposition 2.4].

A3. Proof of Lemma 4.4. Let $\mu \in \mathcal{M}^{e}(f)$. Consider $x \in \Omega$ which is free and satisfies $\lim _{n \rightarrow \infty}(1 / n) \log \left\|D_{x} f^{n} \mid E_{x}^{u}\right\|=\lambda^{u}(\mu)$. The orbit $x, f x, \ldots$ is decomposed into alternative bound and free segments. Applying the expansion estimates in [39, Lemma 2.3, Proposition 2.5] alternatively we have $\left\|D_{x} f^{n} \mid E_{x}^{u}\right\| \geq(2-\varepsilon)^{n}$ if $f^{n} x$ is free. This implies $\lambda^{u}(\mu) \geq \log (2-\varepsilon)$.

Acknowledgments. We thank Renaud Leplaideur, Isabel Rios, Juan Rivera-Letelier, Christian Wolf and anonymous referees for very useful comments. S.S. is partially supported by the CNPq and PRONEX, Brazil. H.T. is partially supported by the Grant-in-Aid for Young Scientists (B) of the JSPS, Grant No.2374012 and the Keio Gijuku Academic Development Funds. This research is partially supported by the Kyoto University Global COE Program. We thank the Mathematics Departments of Kyoto University, the Federal University of Rio de Janeiro, the Pennsylvania State University, l'École Polytechnique Fédérale de Lausanne, and IMPA for their hospitality. 


\section{REFERENCES}

[1] Bedford, E. and Smillie, J.: Real polynomial diffeomorphisms with maximal entropy: tangencies. Ann. Math. 160, 1-25 (2004)

[2] Bedford, E. and Smillie, J.: Real polynomial diffeomorphisms with maximal entropy: II. small Jacobian. Ergodic Theory and Dynamical Systems 26, 1259-1283 (2006)

[3] Benedicks, M. and Carleson, L.: The dynamics of the Hénon map. Ann. Math. 133, 73-169 (1991)

[4] Benedicks, M. and Viana, M.: Solution of the basin problem for Hénon-like attractors. Invent. Math. 143, 375-434 (2001)

[5] Benedicks, M. and Viana, M.: Random perturbations and statistical properties of Hénon-like maps. Ann. Inst. H. Poincaré Anal. Non Linéaire 23, 713-752 (2006)

[6] Benedicks, M. and Young, L.-S.: Sinai-Bowen-Ruelle measures for certain Hénon maps. Invent. Math. 112, 541-576 (1993)

[7] Benedicks, M. and Young, L.-S.: Markov extensions and decay of correlations for certain Hénon maps. Astérisque 261, 13-56 (2000)

[8] Berger, P.: Abundance of one dimensional non uniformly hyperbolic attractors for surface endomorphisms. Preprint. http://arxiv.org/pdf/0903.1473.pdf

[9] Berger, P.: Properties of the maximal entropy measure and geometry of Hénon attractors. Preprint. http://arxiv.org/pdf/1202.2822.pdf

[10] Bowen, R.: Equilibrium states and the ergodic theory for Anosov diffeomorphisms, Springer Lecture Notes in Math. 470 (1975).

[11] Buzzi, J. and Sarig, S.: Uniqueness of equilibrium measures for countable Markov shifts and multidimensional piecewise expanding maps. Ergodic Theory and Dynamical Systems 23, 1383-1400 (2003)

[12] Cao, Y., Luzzatto, S. and Rios, I.: The boundary of hyperbolicity for Hénon-like families. Ergodic Theory and Dynamical Systems 28, 1049-1080 (2008)

[13] Devaney, R. and Nitecki, Z.: Shift automorphisms in the Hénon mapping. Commun. Math. Phys. 67, 137-146 (1979)

[14] Falconer, K.: Fractal Geometry. Mathematical foundations and applications. John Wiley \& Sons, Ltd., Chichester, 1990.

[15] William Feller: An introduction to probability theory and its applications. Vol. I. Third edition. John Wiley \& Sons Inc., New York, 1968.

[16] Hoensch, U. A.: Some hyperbolicity results for Hénon-like diffeomorphisms. Nonlinearity 21, 587-611 (2008)

[17] Jakobson, M.: Topological and metric properties of one-dimensional endomorphisms. Dokl. Akad. Nauk SSSR 243, 1452-1456 (1978)

[18] Kac, M.: On the notion of recurrence in discrete stochastic processes. Bull. Amer. Math. Soc. 53, 10021010, (1947).

[19] Katok, A. and Hasselblatt, B.: Introduction to the modern theory of dynamical systems. Cambridge University Press (1995)

[20] Ledrappier, F. and Young, L.-S.: The metric entropy of diffeomorphisms. Ann. Math. 122, 509-574 (1985)

[21] Leplaideur, R.: Thermodynamic formalism for a family of nonuniformly hyperbolic horseshoes and the unstable Jacobian. Ergodic Theory and Dynamical Systems 31, 423-447 (2011)

[22] Leplaideur, R. and Rios, I.: Invariant manifolds and equilibrium states for non-uniformly hyperbolic horseshoes. Nonlinearity 18, 2847-2880 (2005)

[23] Leplaideur, R. and Rios, I.: On t-conformal measures and Hausdorff dimension for a family of nonuniformly hyperbolic horseshoes. Ergodic Theory and Dynamical Systems 29, 1917-1950 (2009)

[24] Manning, A. and McCluskey, H.: Hausdorff dimension for horseshoes. Ergodic Theory and Dynamical Systems 3, 251-260 (1983)

[25] Mauldin, R. D. and Urbański, M.: Gibbs states on the symbolic space over an infinite alphabet. Isr. J. Math. 125, 93-130 (2001)

[26] Mora, L. and Viana, M.: Abundance of strange attractors. Acta Math. 171, 1-71 (1993) 
[27] Palis, J. and Takens, S.: Hyperbolicity \& Sensitive Chaotic Dynamics at Homoclinic Bifurcations. (Cambridge Studies in Advanced Mathematics Vol 35) Cambridge University Press (1993)

[28] Pesin, Ya.: Families of invariant manifolds which correspond to nonvanishing Lyapunov exponents. Math. USSR-Izv. 10, 1261-1305 (1976)

[29] Pesin, Ya. and Senti, S.: Equilibrium measures for maps with inducing schemes. Journal of Modern Dynamics. 2, 1-31 (2008)

[30] Pesin, Ya., Senti, S. and Zhang, K.: Thermodynamics of towers of hyperbolic type. Preprint. http://arxiv.org/pdf/1403.2989.pdf

[31] Rios, I.: Unfolding of homoclinic tangencies inside horseshoes: hyperbolicity, fractal dimensions and persistent tangencies. Nonlinearity 14, 431-462 (2001)

[32] Rokhlin, V. A.: Lectures on the theory of entropy of transformation with invariant measure. Uspehi Mat. Nauk. 22, 3-56 (1967); English transl., Russian Math. Surveys. 22, 1-52 (1967)

[33] Ruelle, D.: An inequality for the entropy of differentiable maps. Bol. Soc. Brasil. Math. 9, 83-87 (1978)

[34] Ruelle, D.: Thermodynamic formalism. The mathematical structures of classical equilibrium statistical mechanics. Encyclopedia of Mathematics and its Applications, 5. Addison-Wesley Publishing Co.

[35] Ruelle, D.: Ergodic theory of differentiable dynamical systems. Publ. Math. I.H.E.S. 50, 27-58 (1979)

[36] Sarig, O.: Thermodynamic formalism for countable Markov shifts. Ergodic Theory and Dynamical Systems 19, 1565-1593 (1999)

[37] Sarig, O.: Phase transitions for countable Markov shifts. Commun. Math. Phys. 217, 555-577 (2001)

[38] Sarig, O.: Existence of Gibbs measures or countable Markov shifts, Proc. Amer. Math. Soc., 131(6):17511758,2003

[39] Senti, S. and Takahasi, H.: Equilibrium measures for the Hénon map at the first bifurcation. Nonlinearity 26, 1719-1741 (2013)

[40] Sinai, Y.: Gibbs measures in ergodic theory. Uspekhi Mat. Nauk. 27, 21-64 (1972)

[41] Takahasi, H.: Abundance of nonuniform hyperbolicity in bifurcations of surface endomorphisms. Tokyo J. Math. 34, 53-113 (2011)

[42] Takahasi, H.: Prevalent dynamics at the first bifurcation of Hénon-like families. Commun. Math. Phys. 312, 37-85 (2012)

[43] Urbański, M. and Wolf, C.: Ergodic theory of parabolic horseshoes. Commun. Math. Phys. 281, 711-751 (2008)

[44] Wang, Q. D. and Young, L.-S.: Strange attractors with one direction of instability. Commun. Math. Phys. 218, 1-97 (2001)

[45] Young, L.-S.: Statistical properties of dynamical systems with some hyperbolicity. Ann. Math. 147, $585-650(1998)$

[46] Zweimüller, R.: Invariant measures for general(ized) induced transformations. Proc. Amer. Math. Soc. 133, 2283-2295 (2005)

Instituto de Matematica, Universidade Federal do Rio De Janeiro, C.P. 68 530, CEP 21945970, R.J., BRASIL

E-mail address: senti@im.ufrj.br

Department of Mathematics, Keio University, Yokohama 223-8522, JAPAN

E-mail address: hiroki@math.keio.ac.jp 